\title{
Jahrbuch der berufs- und wirtschaftspädagogischen Forschung 2019
}

Eveline Wittmann, Dietmar Frommberger, Ulrike Weyland (Hrsg.)

\section{DGfE}


Jahrbuch der berufs- und

wirtschaftspädagogischen Forschung 2019 


\section{Schriftenreihe der Sektion \\ Berufs- und Wirtschaftspädagogik der Deutschen Gesellschaft für \\ Erziehungswissenschaft (DGfE)}


Eveline Wittmann

Dietmar Frommberger

Ulrike Weyland (Hrsg.)

Jahrbuch der berufs- und

wirtschaftspädagogischen

Forschung 2019

Verlag Barbara Budrich

Opladen • Berlin • Toronto 2019 
Bibliografische Information der Deutschen Nationalbibliothek

Die Deutsche Nationalbibliothek verzeichnet diese Publikation in der Deutschen Nationalbibliografie; detaillierte bibliografische Daten sind im Internet über

http://dnb.d-nb.de abrufbar.

(C) 2019 Dieses Werk ist bei der Verlag Barbara Budrich GmbH erschienen und steht unter der Creative Commons Lizenz Attribution-ShareAlike 4.0 International (CC BY-SA 4.0): https://creativecommons.org/licenses/by-sa/4.0/.

Diese Lizenz erlaubt die Verbreitung, Speicherung, Vervielfältigung und Bearbeitung bei Verwendung der gleichen CC-BY-SA 4.0-Lizenz und unter Angabe der UrheberInnen, Rechte, Änderungen und verwendeten Lizenz. www.budrich.de

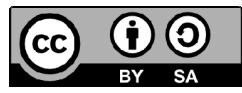

Dieses Buch steht im Open-Access-Bereich der Verlagsseite zum kostenlosen Download bereit (https://doi.org/10.3224/84742330).

Eine kostenpflichtige Druckversion (Print on Demand) kann über den Verlag bezogen werden. Die Seitenzahlen in der Druck- und Onlineversion sind identisch.

$$
\begin{array}{ll}
\text { ISBN } & 978-3-8474-2330-0 \text { (Paperback) } \\
\text { eISBN } & 978-3-8474-1351-6 \text { (eBook) } \\
\text { DOI } & 10.3224 / 84742330
\end{array}
$$

Umschlaggestaltung: Bettina Lehfeldt, Kleinmachnow - www.lehfeldtgraphic.de Druck: Books on Demand GmbH, Norderstedt Printed in Europe 


\section{Inhaltsverzeichnis}

Aktuelle theoretische und empirische Perspektiven der Berufs- und Wirtschaftspädagogik

\section{Teil I: Berufsbildungs-, organisations- und professionstheoretische Perspektive}

Franz Kaiser und Thilo J. Ketschau

Die Perspektive kritisch-emanzipatorischer Berufsbildungstheorie als

Widerspruchsbestimmung von Emanzipation und Herrschaft

Marc Egloffstein, Tobias Heilig und Dirk Ifenthaler

Entwicklung eines Reifegradmodells der Digitalisierung für Bildungsorganisationen

Karin Reiber, Ulrike Weyland und Eveline Wittmann

Professionalisierung des schulischen Bildungspersonals in den Gesundheits- und Pflegeberufen - Zwischenfazit eines berufs- und wirtschaftspädagogischen Sonderweges

\section{Teil II: Berufsdidaktische Perspektive}

Tobias Kärner, Hannes Reinke, Anja Frim und Karin Heinrichs Innere Differenzierung im Unterricht mit jugendlichen Asylsuchenden und Geflüchteten aus der Sicht von Lehrpersonen

Bärbel Wesselborg, Ulrike Weyland und Marc Kleinknecht

Entwicklung eines fachdidaktischen Kategoriensystems zur Analyse des kognitiv-aktivierenden Potenzials von Aufgaben - ein Beitrag zur Unterrichtsqualitätsforschung in der beruflichen Fachrichtung Pflege

Julia Schultheis und Carmela Aprea

Entwicklung und Validierung eines Schemas zur Evaluation von Serious

Games im Kontext von Financial Literacy

Tim Stanik

Mikrodidaktische Planungen von Trainerinnen und Trainern in der betrieblichen Weiterbildung 


\section{Aktuelle theoretische und empirische Perspektiven der Berufs- und Wirtschaftspädagogik}

Mit Blick auf die thematische und methodische Vielfalt der deutschsprachigen Berufsbildungsforschung - diese bearbeitet z. B. Fragen der Institutionalisierung beruflicher Bildung, der Didaktik und Methodik des beruflichen Lernens, der Kompetenzmodellierung und Kompetenzmessung, der Lehrerbildungsforschung, der beruflichen Aus- und Weiterbildung, der historischen Berufsbildungsforschung sowie der Hochschuldidaktik - entschloss sich die Sektion für Berufs- und Wirtschaftspädagogik der Deutschen Gesellschaft für Erziehungswissenschaft (DGfE) im Jahr 2012, ein ,Jahrbuch der berufs- und wirtschaftspädagogischen Forschung" herauszugeben. Damit soll der Breite und Tiefe der Forschung und Theoriebildung in der Berufs- und Wirtschaftspädagogik besser Rechnung getragen werden. Die Aufgabe des Jahrbuchs besteht darin, die im Rahmen der Sektionstagungen geführten Diskussionen zu dokumentieren und damit einen Einblick in den aktuellen Stand der Berufsbildungsforschung zu geben. Mit dem hier vorgelegten fünften Jahrbuch sollen die Arbeiten der Sektion Berufs- und Wirtschaftspädagogik erneut einer interessierten Öffentlichkeit zugänglich gemacht werden.

Die im September 2018 an der Johann-Wolfgang-von-Goethe-Universität Frankfurt durchgeführte Jahrestagung der Sektion Berufs- und Wirtschaftspädagogik der DGfE erfreute sich einer sehr guten Resonanz. Das aktuelle Jahrbuch präsentiert aus der Bandbreite der Vorträge und Symposien insgesamt sechs Beiträge, die wie gewohnt einem doppelten Review-Verfahren unterzogen wurden.

Das Jahrbuch ist in diesem Jahr in zwei Teile untergliedert. In einem ersten Abschnitt widmet sich das Jahrbuch zunächst ausgewählten berufsbildungs-, organisations- und professionstheoretischen Fragestellungen. Der zweite, umfangreichere Abschnitt thematisiert vorrangig mit einer empirischen Methodologie didaktische Fragen sowohl der schulischen als auch der betrieblichen Ausbildung.

Im ersten Abschnitt wenden sich zunächst Franz Kaiser und Thilo J. Ketschau der „Perspektive kritisch-emanzipatorischer Berufsbildungstheorie als Widerspruchsbestimmung von Emanzipation und Herrschaft" zu. In ihrem Beitrag, den die Autor*innen dem jüngst verstorbenen Kollegen Wolfgang Lempert widmen, nehmen sie den Verlust soziologischer Perspektiven in der berufspädagogischen Diskussion zum Ausgangspunkt, um die Frage nach dem Sinn beruflichen Handelns und von Wirtschaft im Kontext einer marktorientierten Verwertungslogik und der Digitalisierung der Arbeitswelt aufzuwerfen. Trotz der curricular geforderten Sach- und Selbstreflexion mangele es an der Suche nach Verständnis der eigenen Berufsrolle in der Gesellschaft, die Betrachtung ihrer historischen Bedingtheiten und der kritischen Reflexion der 
Maßstäbe für die Unterwerfung unter oder die Befreiung von diesen Bedingtheiten. Anzulegen sei eine Berufsbildungstheorie sowohl auf die Identifizierung eines beruflichen Bildungsideals als auch auf das Aufzeigen struktureller, begrifflicher und anderer Barrieren seiner Erreichung. Gegenwärtige institutionelle Rahmungen des Berufsbildungssystems wiesen mit der Kompetenzorientierung die Pflichterfüllung des jungen Menschen als pädagogischen Kern aus und vernachlässigten Selbstentfaltung und Solidarität. Die Glaubwürdigkeit einer kritisch-emanzipatorischen Berufsbildungstheorie hänge allerdings von ihrer Konkretisierung für die berufspädagogische Praxis und ihrem Bezug zu gesellschaftlichen Entwicklungen ab, wie der Nachhaltigkeits- und der Digitalisierungsthematik. Widersprüche, latente Widerstände und Gestaltungsoptionen in der Berufs- und Arbeitspraxis verlangten dementsprechend nach wissenschaftlicher Eruierung und kategorialer Fundierung.

Marc Egloffstein, Tobias Heilig und Dirk Ifenthaler entwickeln in ihrem organisationstheoretisch ausgerichteten Beitrag ein Reifegradmodell, das als Bezugsbasis für die Begleitung und Gestaltung von Digitalisierungsprozessen in Bildungsorganisationen dienen und Indikatoren für die „digitale Reife“ einer Bildungsorganisation enthalten soll. Die Modellierung von Reifegraden ist den Autoren zufolge vorrangig dem wirtschaftsinformatischen Kontext entlehnt und bezieht sich üblicherweise auf Dimensionen und Reifestufen; Referenzmodelle für Organisationen der beruflichen Bildung existieren bislang nicht. Die Autoren fügen den Dimensionen üblicher Reifegradmodelle - u. a. Ausstattung/Technik, Führung/Strategie, Organisation, Mitarbeitende, Kultur - die Dimension digitales Lehren und Lernen hinzu und weisen auf die wechselseitige Beeinflussung der Dimensionen hin. Die Autoren konkretisieren dieses Modell exemplarisch anhand einer Bildungorganisation, und verdeutlichen fokussierend auf die Dimension Mitarbeiter die vorgenommene Stufendifferenzierung.

Der professionstheoretisch zugeschnittene Beitrag von Karin Reiber, Ulrike Weyland und Eveline Wittmann nimmt einige Fäden dieser Positionierung auf, wozu er drei Einzelbeiträge eines Symposiums der Sektionstagung in einen kontextualisierenden Zusammenhang stellt. Vor dem Hintergrund der weitgehend als Sonderweg zu bezeichnenden Entwicklung nicht nur der Gesundheits- und Pflegeberufe, sondern insbesondere auch der Professionalisierung ihres schulischen Bildungspersonals wird hier mit Fokus auf die berufliche Fachrichtung Pflege gefragt, wie sich im Spannungsgefüge von verspäteter Professionalisierung einerseits und ökonomischen und sozialen sowie technischen Entwicklungen andererseits die professionelle Wissensbasis des schulischen Bildungspersonals entwickelt, wobei auf letztere, auch angesichts der kontinuierlichen Veränderungen in Ermangelung einer entsprechenden empirischen Befundlage, aus der institutionellen und strukturellen Rahmung geschlossen wird. Einerseits werden Akademisierungstendenzen der Ausbildung 
des schulischen Bildungspersonals sowie die verstärkte disziplinäre Integration des gesundheits- und pflegeberuflichen Bildungsdiskurses in den berufsund wirtschaftspädagogischen Diskurs konstatiert, andererseits beinhaltet der weiterhin bestehende Sonderweg angesichts der historischen Gender-Codierung des Berufs die Gefahr einer Deregulierung und Abwertung, z.B. im Kontext der Digitalisierung des Berufsfeldes. Zwar werden gerade diesbezüglich Modernisierungslücken in Bildungsmaßnahmen für das schulische Bildungspersonal der Pflegeberufe partiell angegangen, insgesamt erweist sich diese akademische Ausbildungslandschaft jedoch als fortwährend fragmentiert. Abzuwarten bleibt, inwieweit der neue Fachqualifikationsrahmen Pflegedidaktik, der die angesprochene Problematik selbst möglicherweise nur teilweise aufgreift, zu einer Vereinheitlichung führen kann und insbesondere auch Barrieren auf der Ebene der Umsetzung in individuelle Handlungslogiken überwunden werden können.

Der zweite Abschnitt des Jahrbuchs bezieht sich auf berufsdidaktische Perspektiven der Berufs- und Wirtschaftspädagogik. Im ersten Beitrag dieses Abschnitts gehen Tobias Kärner, Hannes Reinke, Anja Frim und Karin Heinrichs im Rahmen einer explorativen Interviewstudie mit 16 Proband*innen der Sicht von Lehrpersonen nach, wobei ihr Beitrag nach Differenzierungsaspekten, -kriterien und -barrieren sowie Aus- und Fortbildungserfordernissen von Lehrkräften im Zusammenhang mit der Inneren Differenzierung bei der Unterrichtung von jugendlichen Asylsuchenden und Geflüchteten fragt. Aus Sicht der Lehrkräfte handelt es sich bei der Inneren Differenzierung demnach um ein sehr voraussetzungsreiches Konzept, für das auch substanzielle Ausbildungsdefizite der Lehrkräfte artikuliert werden. Gerade bei der Beschulung jugendlicher Asylbewerber*innen und Flüchtlinge erscheint die erforderliche prozessbegleitende Diagnostik angesichts der zu verarbeitenden Informationsmenge anfällig für Urteilsfehler und damit einhergehende Fehleinschätzungen; diesbezügliche Unterstützungssysteme werden von den Autor*innen des Beitrags als erforderlich herausgestellt.

Zwei Beiträge befassen sich mit der Entwicklung und Validierung von Evaluierungsschemata. Das von Bärbel Wesselborg, Ulrike Weyland und Mark Kleinknecht entwickelte fachdidaktische Kategoriensystem bezieht sich auf die Analyse des kognitiv-aktivierenden Potenzials von Aufgaben in der beruflichen Fachrichtung Pflege. Das Erfordernis einer fachdidaktischen Professionalisierung wird hier gerade auch vor dem Hintergrund der Heterogenität der Ausbildungsstrukturen des schulischen Bildungspersonals in dieser Fachrichtung gesehen. Das Instrument selbst wurde inhaltsanalytisch aus existierenden Kategoriensystemen anderer fachdidaktischer Kontexte abgeleitet, adaptiert und verfeinert sowie auf Reliabilität überprüft. Das entwickelte Schema umfasst die Kategorien Wissensart, kognitiver Prozess, Wissenseinheiten, Offenheit, Lebensweltbezug, sprachlogische Komplexität und Repräsentationsformen. Perspektivisch kann das entwickelte Schema den Autor*innen zufolge 
als valide Grundlage für die Erfassung und Erforschung des kognitiven Potenzials von Aufgaben in der beruflichen Fachrichtung Pflege herangezogen werden.

Im Fall des Beitrags von Julia Schultheis und Carmela Aprea geht es darum, ein Kategorienschema für die Prüfung von Serious Games im Kontext von Financial Literacy zu entwickeln. Vor dem Hintergrund von Finanzkrisen sowie der Gefahr individueller Schuldenfallen durch zunehmend komplexere Finanzprodukte einerseits und der mutmaßlichen Lernrelevanz von Serious Games andererseits liegt der Fokus auf der didaktischen Gestaltung dieser Spiele. Im Rahmen eines Input-Process-Outcome-Modells gamebasierten Lernens fokussiert der Beitrag die Input-Seite der Lerninhalte und Spieleigenschaften und rekurriert inhaltlich auf die konkret ausdifferenzierten Kompetenzdimensionen ,persönliches Finanzmanagement" sowie ,ökonomischer und gesellschaftlicher Kontext". Gamebezogene Gestaltungskriterien werden auf Basis einer Analyse der einschlägigen Literatur identifiziert. Im Rahmen eines designbasierten Forschungsansatzes entlang der Schritte Bedarfsanalyse, Rasterentwicklung, Rasterüberprüfung und anhand eines Samples von zehn Spielen wurde das Evaluationsschema entwickelt. In einer weiteren Studie soll das Raster bei der Analyse eines umfassenden Katalogs an Serious Games zur Financial Literacy zum Einsatz kommen.

Der den Band abschließende Beitrag von Tim Stanik behandelt anhand einer qualitativen Studie die Frage, wie Trainer*innen in der betrieblichen Bildung Unterricht vorbereiten und mikrodidaktisch planen. Konkret geht es um das planerische Vorgehen und das Treffen didaktischer Entscheidungen sowie die wechselseitige Verzahnung von Planungsentscheidungen. Anhand eines kleinen heterogenen Samples betrieblicher und außerbetrieblicher Weiterbildner wurden, basierend auf einer Grounded Theory-Methodologie, problemzentrierte Interviews ausgewertet. Im Ergebnis differenziert der Verfasser drei Planungsebenen: eine soziale Prozessebene, die der Auftragsklärung dient, eine kognitive Strategieebene, die im engeren Sinne der didaktisch-methodischen Planung gewidmet ist, und eine materiale Planungsebene, die im Sinne der betrieblichen Handlungslogik der Sicherung der Qualität des Produkts Training bzw. des Outcomes dient. Die empirischen Auswertungen verdeutlichen auch, wie sich Überlegungen von Trainer*innen in existierende betriebliche Trainingslogiken einpassen.

Der Vorstand der Sektion Berufs- und Wirtschaftspädagogik der DGfE dankt sehr herzlich Frau Prof. Dr. Eveline Wuttke und Herrn Prof. Dr. Gerhard Minnameier (Johann-Wolfgang-von-Goethe-Universität Frankfurt) sowie den dortigen Mitarbeiter*innen für die Ausrichtung der Sektionstagung. Unser Dank gilt zudem den Gutachter*innen, die die eingegangenen Beiträge in akribischer Manier unter die Lupe nahmen und viele konstruktive Hinweise zur 
Verbesserung der Texte lieferten. Schließlich danken wir Frau Anya Prommetta und Frau Leonie Seibold für die engagierte Unterstützung bei der redaktionellen Bearbeitung der Beiträge.

München, Osnabrück und Münster, im Juli 2019

Eveline Wittmann, Dietmar Frommberger und Ulrike Weyland 



\section{Die Perspektive kritisch-emanzipatorischer Berufsbildungstheorie als Widerspruchsbestimmung von Emanzipation und Herrschaft ${ }^{1}$}

Franz Kaiser und Thilo J. Ketschau

\section{Emanzipation und Gesellschaftskritik als Momente der Berufsbildung - ein Plädoyer vor dem Hintergrund aktueller Herausforderungen}

Die Ideen von Erziehung als Mittel der Emanzipation und Bildung als Medium von Gesellschaftskritik sind bereits mit Anna Siemsens Entgegnungen zu Kerschensteiner (Büchter \& Kipp 2009), aber breiter seit den Arbeiten von Herwig Blankertz und Wolfgang Lempert als Prämissen der Berufsbildung offeriert worden. In ihrer konkretisierten Form als kritisch-emanzipatorisches Paradigma konnten sie zwar nie Prioritätscharakter für die Berufs- und Wirtschaftspädagogik erlangen, doch auch die massive Rezeption der sogenannten realistischen Wende, die sich im Boom entpolitisierter und teilweise enttheoretisierter empirischer Berufsbildungsforschung niederschlug, hat nicht zu ihrem völligen Verschwinden geführt (Schapfel-Kaiser 2003; Büchter 2017; Ketschau 2018). Geißler (1974) entwickelt mit Bezug auf Lempert und rekurrierend auf die kritische Theorie der Frankfurter Schule sowie Habermas' Theorie kommunikativen Handelns einen pädagogisch orientierten Gestaltungsansatz beruflicher Bildung im Sinne der Entfaltung kritischer Kompetenz. Kipp (1992) setzt sich über die Betrachtung der nationalsozialistischen Berufsbildungspraxis mit handlungsorientierten Konzepten der beruflichen Bildung auseinander. Lisop und Huisinga (2004) entwickeln mit der arbeitsorientierten Exemplarik eine gesellschaftstheoretisch orientierte Didaktik, welche Herrschaftsmechanismen bspw. in technischer Normierung, Internationalität und betrieblicher Organisation erkennbar macht.

An der Aktualität ihres Gegenstands mangelt es einer kritisch-emanzipatorischen Berufsbildung auch im 21. Jahrhundert nicht: Die zunehmende Durchdringung der Gesellschaft durch neoliberale Ideologie, eine schlei-

1 Wir danken den Diskussionsbeiträgen auf dem Symposium „Emanzipation und Gesellschaftskritik als Momente der Berufsbildung" in Frankfurt für ihre kritischen und anregenden Beiträge, insbesondere den Kolleginnen Karin Büchter und Marianne Friese. Der Beitrag ist Wolfgang Lempert gewidmet. 
chende Abkehr von der Unbestreitbarkeit der Menschenrechte durch eine irrationale Angst vor Überfremdung, der Verlust des Subjekts in einer Gesellschaft der Singularitäten oder auch die zunehmend egalitäre Einstellung zu demokratischer Teilhabe, lassen eine Pathologie der Gesellschaft vermuten, welche die kritische Theorie und ihre Ableger seit vielen Jahrzehnten zu dekuvrieren suchen. $^{2}$

Wenn berufliches Handeln und berufliche Bildung dem entgegenwirken und Individuen zur kritischen Teilhabe und Gestaltung ihres Lebens- und Arbeitskontexts befähigen und darüber hinaus zur Einordnung des eigenen Handelns in die großen Kontexte ermutigen soll, dann muss die Berufspädagogik sich den Spannungsfeldern und Widersprüchen widmen, die sie selbst mitverursacht - und sie zuvorderst wahrnehmen. „Um die Menschen zu lieben muss man sehr stark hassen, was sie unterdrückt" formulierte Jean-Paul Sartre (zitiert nach Zeigler \& Gebauer 2018, 5), was man durchaus als Anspruch an alle Pädagogiken verstehen kann: die Edukand*innen nicht bloß auf die Alltäglichkeit vorzubereiten, sondern sie für die Überwindung dessen, was ihnen als Schlechtem begegnet, zu wappnen, anstatt sich diesem zu ergeben.

Zeitgleich durchdringt die von kritischer Theorie als Quelle der Barbarei identifizierte kapitalistische Logik alle Nischen des Lebens. Die Kategorien der Effizienz und der Nützlichkeit sind Maßstab für alle Bildungsinstitutionen und Arenen gesellschaftlichen Handelns. Die Mechanismen der Kontrollgesellschaft verursachen die Verheißung evidenter Bildungspolitik bei gleichzeitigem katastrophalem Scheitern, wie Allais (2017) eindrucksvoll an den Wirkungen nationaler Qualifikationsrahmen und outputorientierter Bildungspolitik zeigen kann.

Und es schüren nicht nur die digitalisierte Veränderung der Arbeitswelt und die Sorge um den Fortbestand lebendiger Arbeit (Pfeiffer 2014) die Furcht vor dem Verlust der Gemeinschaft, sondern auch designte Gouvernance mit gezielter Form die Angst (Milev 2013). Dieser erleichterten Regierbarkeit hat sich Pädagogik (Dobmeier 2018) und kritische Berufsbildung entgegenzustellen und zum Widerspruch zu befähigen (Kaiser 2017). Das stellt die Orientierung der Berufs- und Wirtschaftspädagogik vor grundlegende Probleme, welche an ihrem Selbstverständnis rütteln sollten, und die u. a. Lempert (2008) mit zwei Fragen in der ZBW pointierte: Wie soll vor einer Wirtschaftsordnung pädagogisch gehandelt werden, die ,trügerisch nur den Tauschwert (alias Täuschwert) produzierter Güter und erwiesener Dienste registriert“" (Lempert

2 So verweist kritische Bildungstheorie immer wieder auf den gesellschaftlichen Zusammenhang individuellen Leidens, das zum Gegenstand der Pädagogik zu machen ist. „Die Demütigung im Vorhof von Überproduktion und -genuß unter Quarantäne gestellt zu sein, wird überboten von der Erfahrung der Konkurrenz mit Polen, Rumänen, Türken und Schwarzafrikanern um den Zugang zu den Wohlstandszitadellen. In den faschistischen Gewaltreaktionen werden die Facetten erkennbar, mitgeschleppte Aggressivität vernachlässigter Sepsis, in der die verdrängte Geschichte aus ihrer Latenz hervorbricht.“ (Koneffke 1995, 54). 
2008, 467) und angesichts drohenden Untergangs nicht zu Selbstbeschränkung ermutigt, sondern ,vielmehr die Menschen eher unzufriedener und damit noch gieriger macht" (ebd.)? Welche Lehrkraft kann guten Gewissens an der Integration von Jugendlichen und Erwachsenen in diese Ordnung mitwirken und welche Hochschullehrer*innen können dieses Reproduktionsgebahren verantworten? Kritische Berufsbildung muss zu solcher Infragestellung und anschließendem Handeln ermutigen.

Demzufolge ist nicht nur der gesellschaftliche Sinn beruflichen Handelns (Graeber 2018), sondern auch der Sinn von Wirtschaft (Nell-Breuning 1975) als gesellschaftlicher Teilbereich, in dem berufliches Handeln überwiegend stattfindet, sowie die mit ungebändigter Gewinnmaximierung einhergehende Expansion (Welskopp 2018) kapitalistischer Logik in öffentlichen Bildungskontexten und -institutionen zu thematisieren. Stand in den Anfängen der Berufsbildungstheorie der Beruf als Zugang zur Kultur der Gesellschaft und zum individuellen Wesen im Fokus (Müllges 1967), so scheint sich das Interesse an der beruflichen Sozialisation und der Wechselwirkung zwischen Subjekt und Beruf (Beck, Brater \& Daheim 1980) in der Disziplin mit der Abkehr von der Soziologie und der Zuwendung zu psychometrischen Modellen verflüchtigt zu haben. Die Suche nach Sinn und Beitrag, welche das berufliche Handeln zum gesellschaftlichen Wohlstand in sich birgt, scheint nicht mehr Teil der beruflichen Bildung $\mathrm{zu} \operatorname{sein}^{3}$ und dies trotz des Wissens darum, dass

sich die Trennung von Politik und Beruf nicht durchführen läßt, da der Gewerbelehrer seinen Schülern die politische Bildung nicht gleichsam an der beruflichen Wirklichkeit vorbei vermitteln kann, sondern nur durch soziologische Analyse und Kritik. Wenn er dem Jugendlichen helfen will, mündiger Bürger zu werden, dann muß er ihnen die Betriebsverfassung und das Wirtschaftssystem der Bundesrepublik als Kompromißlösung von Gruppenkonflikten darstellen, die im Widerspruch stehen zu wichtigen Prinzipien unseres Grundgesetzes und zu den Interessen der Arbeitnehmer, d. h. der überwiegenden Mehrzahl der Beschäftigten, und die darum der Änderung bedürfen (Lempert 1971, 96).

Folglich sind die Zusammenhänge beruflichen Handelns zur gesellschaftlichen Wirklichkeit der Arbeits- und Lebenswelt an dieser exemplarisch zu verdeutlichen (Lisop \& Huisinga 2004), denn nur so sind in der beruflichen Bildung „...der dafür vorausgesetzte gesellschaftliche Implikationszusammenhang und der Kontext individueller Lebensführung thematisierbar.“ (Kutscha 2008, 7).

3 Bei der Entwicklung der Klassifikation der Berufe in Deutschland zum Jahr 2010 zeigte sich, dass in den vorangegangenen Klassifikationen der Berufsausbildung die Frage nach der gesellschaftlichen Bedeutung eines Berufes durchaus noch von Relevanz für das Verwandschaftsverhältnis in der Klassifikation und der Berufsberatung und Arbeitsvermittlung war. Die neue Klassifikation misst hingegen nur noch die Ähnlichkeit und Häufigkeit von Teiltätigkeiten zur Bestimmung des Verwandschaftsgrades von Berufen (KldB 2010). 
Die mit beruflicher Ausbildung einhergehende Vorbereitung auf eine dienende Funktion (Stütz 1970) steht demgegenüber traditionell im Fokus pädagogischer Bemühungen; weniger dagegen emanzipative, strukturkritisch reflektierende Fähigkeiten. Wird Reflexion als pädagogischer Begriff und Anspruch avanciert, reduziert sie sich meist auf Selbst- und Sachreflexion und steht dann synonym für die Selbststeuerung des Individuums, orientiert an den Bedürfnissen des Marktes, dessen Primat unhinterfragt bleibt (Schapfel-Kaiser 1998). Es fehlt eine darüber hinausgehende Wesensreflexion, also die Suche nach dem Verständnis der eigenen Rolle als Berufstätiger in der Gesellschaft, der Betrachtung der Bedingungen und historischen Bedingtheit dieser Rolle und vor allem der Frage danach, wie und warum sich die Edukand*in selbst zu ihrem Guten oder Schlechten in diese einfügt. Käme diese Frage in einem Bildungsprozess der miteinander geteilten Reflexion zum Tragen, so könnte ,eine allgemeine Erkenntnis wachsen, die sich aus individueller, sinnlicher Erfahrung speist: daß wir alle Opfer sind mit der Aussicht auf Freiheit, daß wir alle schon freigesetzt sind inmitten der Determination“ (Pongratz 1995, 35). Die Reflexion des Preises der Unterwerfung an das Gegebene in der beruflichen Ausbildung, um Mitglied der Community of Practice zu werden, ist in einer Weise zum Gegenstand zu machen, dass die Entscheidung über dessen Höhe den Auszubildenden verfügbar wird. Das ist sicherlich nicht hinreichend für eine auf Emanzipation abzielende Bildungsbemühung, als ihre Grundlage ist es aber notwendig.

Die folgende Argumentation nähert sich dem Kern der kritischen Berufsbildungstheorie bereits progressiv und konkret. Sie zeigt anschließend an die Verdeutlichung der Relevanz entsprechender Überlegungen, wo die Perspektiven der Fortentwicklung der Theorie liegen, die angesichts der seit ihrer Blütezeit veränderten gesellschaftlichen Bedingungen und ihres Bedeutungsverlusts dringend geboten scheint. Daran anknüpfend schließt sich die theoretische Erläuterung des Spannungsfelds an, das sich vor dem Antagonismus der Verwertungsansprüche des Marktes gegenüber dem Subjekt und dessen Recht und Drang nach Selbstentfaltung und Befreiung aufbaut. Der argumentative Wert der Auseinandersetzung mit diesem Gegensatz begründet sich in dessen konstitutiver Natur für die kritisch-emanzipatorische Theoriefolie. Denn der Antagonismus von Emanzipation, verstanden als Ausdruck der von Bildung entfalteten Menschenwürde ${ }^{4}$ gegenüber einer pädagogisierten Nützlichkeit, gedeutet als Ausdruck kapitalistisch notwendigen Herrschaftsbemühens, muss

4 Emanzipation ist kein theoretisch eindeutig gefasster Begriff. Seine allgemeinste Bedeutung ist die Aufhebung menschlicher Fremdbestimmung. Das bezieht sich politisch auf die Beseitigung von einseitigen Abhängigkeitsverhältnissen, Benachteiligung und Ungerechtigkeit und damit auf die Veränderung gesellschaftlicher Beziehungen (Lempert 1974, 27f.). „Insofern sind alle Emanzipationstendenzen egalitär. Sie zielen auf die Gleichheit der Rechte und Pflichten aller Gesellschaftsmitglieder" (a. a. O., 29) ab. 
durch Erziehende und Edukand*innen gleichermaßen wahrgenommen werden, um ein Bewusstsein der heimlich ideologisierten Ausschließlichkeit der Verwertbarkeit zu schaffen, die es letztlich zu überwinden gilt - und damit die Emanzipation als pädagogischen Sinn schrittweise wieder als etwas Mögliches begreifbar machen kann.

Aus diesen Überlegungen ergeben sich bereits erste Prämissen, welche einer erneuten Aufarbeitung des Berufsbildungsbegriffs unter der Folie kritischemanzipatorischer Theorie zugrunde liegen können.

\section{Fortentwicklung des Verständnisses kritisch- emanzipatorischer Berufsbildungstheorie}

Soll das gesellschaftskritische Moment wieder deutlicheren Zugang zur Berufs- und Wirtschaftspädagogik finden, so ist das mit der Arbeit an einer Weiterentwicklung und Rekonstruktion der kritisch-emanzipatorischen Berufsbildungstheorie ein explizit normatives Unterfangen. Die wissenschaftliche und pädagogische Arbeit unter der Folie kritisch-emanzipatorischer Berufsbildungstheorie ist immer ausdrücklich an die Prämisse gebunden, dass Berufsund Wirtschaftspädagogik nicht nur deskriptiv und konformistisch sein darf, denn um die wertende Erkenntnis des Erstrebens- und Vermeidenswerten kommt sie zur Bestimmung ihres Sinns nicht umhin (Ketschau 2018). Damit fordert kritisch-emanzipatorische Berufsbildung von der Disziplin nicht nur deskriptiv den Zustand ihres Gegenstands zu bestimmen und die effizientere Gestaltung ihrer Instrumente und Strukturen anzustreben. Sie erfordert die Auseinandersetzung mit Sinn, Normen und Menschenbild bereits innerhalb der Disziplin, um sich der eigenen Rolle und Wirkung bewusster zu werden und damit einen progressiven und kritischen Horizont zu entfalten.

Eine konsequente kritische Berufsbildungstheorie muss, um den Anspruch ihrer Semantik einlösen zu können, zwei Perspektiven gleichermaßen beleuchten, die in der Fortentwicklung einer solchen Theorie sukzessive-interdependent zueinander entwickelt werden müssen. Einerseits gilt es grundlegend das Emanzipatorische als den positiven Aspekt dieser Theorie herauszuarbeiten: Wie soll Berufsbildung sein? Welches Ideal kann und muss sie am Auszubildenden vollbringen? Wie kann das ermöglicht werden? Was bedeutet Emanzipation durch Beruf und Arbeit und was nicht? Andererseits zeigt sich, als dialektisches Gegenstück und logisches Komplement, der negative Aspekt als kritische Betrachtung von Kultur und Gesellschaft. Es muss offengelegt werden, welche präsenten Ideen, Begriffe, Strukturen und Praktiken ${ }^{5}$ in der Berufs- und

5 Mit anderen Worten: Formen und Denkfiguren der Herrschaft. 
Wirtschaftspädagogik dem Streben nach einem emanzipatorischen Ideal und seiner Umsetzung als Barrieren entgegenstehen, deren Überwindung damit Sinn des Pädagogischen wird. Dieser positive und dieser negative Aspekt definieren den kategorialen Rahmen einer kritischen Berufsbildungstheorie (Ketschau 2018), der für viele Suchen Raum gewährt: Mündigkeit, Gerechtigkeit, Kapitalismuskritik, Kulturkritik, Subjektorientierung, Gestaltungsmacht, Heteronomiekritik. Ihre Gemeinsamkeit zielt auf die Überwindung vorgegebener Allgemeinheit, denn „Anpassung an Gegebenes ist nicht Sache der (beruflichen) Bildung" (Kaiser 2016, 193). Und es ist dieses grundlegende Verständnis, welches als Kern einer kritisch-emanzipatorischen Programmatik „nicht nur die Berufsbildung vom instrumentellen Charakter lossagt, sondern sie dazu verpflichtet, ihre Strukturen und ihren Kontext dort zu demaskieren, wo die Entfaltung des Individuums als Prämisse aufgegeben wurde“ (Ketschau 2018, 98). So weist auch Büchter in ihrer Diskussion des historischen Verhältnisses von allgemeiner und beruflicher Bildung auf die Notwendigkeit der Reflexion der Ökonomie hin. Berufliche Bildung bedarf ,... einer gemeinsamen (selbst-) kritischen Auseinandersetzung damit, wie und in welcher Weise Denken und Handeln von welchen ökonomischen Kategorien geleitet sind, und welches Verständnis einer humanen und gemeinnützigen Ökonomie maßgeblich sein kann“ (Büchter 2017, 39).

Grundlegend neben einer kategorialen Eingrenzung bleibt die Frage nach dem Wesen einer emanzipatorischen Berufsbildung, das einem Ideal ihres Anspruchs voraus gehen muss, will sie sich nicht in metaphysischer Unverbindlichkeit verlieren. Es findet sich im Raum zwischen allgemeiner und spezieller Bildung, denn ,weder gibt es eine politisch-gesellschaftlich keimfreie Allgemeinbildung, die den Menschen vor und unabhängig von aller Ausbildung für gesellschaftliche Zwecke zum Menschen machen könnte [...], noch sind die Inhalte der Berufsausbildung schon als solche Folterzangen zur Selbstentfremdung des Menschen“ (Blankertz 1983, 809). Berufsbildung wird zur im besten Sinne der Aufklärung verbundenen Bildung, sofern sie nicht „Erlösungsvision durch Bildung im Niemandsland des Gedankens" (Heydorn 1971, 200) und nicht „Zurichtung auf die Produktion in der Steuerung der Herrschenden“ (Kaiser 2016, 191) ist. Damit wird verlangt, dass Bildung nicht zu abstrakt und entkoppelt von der realen Welt und ihren Bedingungen sein darf, genauso wenig sich aber auf das in der Arbeit verwertbare Sachwissen und berufliche Kompetenzen reduzieren soll. Vielmehr müssen beide Extreme nicht nur aneinander bereichert, sondern die Legierung gefunden werden, die eine Form von Bildung wird, welche auf die produktive Reflexion der Gesellschaft durch das an ihr partizipierende Individuum abzielt. Dieser Anspruch soll das Streben emanzipatorischer berufsbildungsphilosophischer Erkenntnisbemühungen bestimmen. 
Die Glaubwürdigkeit kritisch-emanzipatorischer Berufsbildungstheorie hängt nicht nur von ihrer inneren Logik ab. Sie fußt auch auf ihrer Konkretisierung für die berufspädagogische Praxis (vgl. Kaiser 2019) und ihrem Bezug zu aktuellen gesellschaftlichen Entwicklungen, an denen die Fortentwicklung ausgerichtet sein sollte und die als Gegenstände den empirischen Forschungsansätzen der 1980er Jahre teilweise noch nicht vorlagen. So können der funktionalistischen Erschließung der Berufsbildung für nachhaltige Entwicklung, bspw. im Rahmen der Modellversuchsforschung (bspw. Rebmann, Schlömer, Feldkamp, Jahncke, \& Lüllau 2017), eine gesellschaftskritische pädagogische Position, wie die von Kehren (2016) formulierte, entgegengehalten werden. Digitalisierung wäre nicht nur hinsichtlich ihrer zunehmenden Bedeutung als Facette beruflicher Handlungskompetenz zu untersuchen, sondern auch nach dem, durch sie provozierten, Verlust von Selbstkontrolle, wie es Lankau (2015) im pädagogischen Kontext diskutiert hat. Auch die Problematik der Instrumentalisierung von Large-Scale-Assessments wie PISA als undemokratisches Herrschaftsinstrument, thematisiert bspw. durch Dammer (2015), gilt es vor der Expansion quantitativer Diagnostik in der Berufs- und Wirtschaftspädagogik für die Berufsbildung aufzuarbeiten.

\section{Verwertungslogik und Spannungsfeld}

Humboldt prägte den Bildungsbegriff, indem er Bildung als „Anregung aller Kräfte eines Menschen [fasste], damit sich diese über die Aneignung der Welt [...] entfalten und zu einer sich selbst bestimmenden Individualität oder Persönlichkeit führen, die in ihrer Einzigartigkeit die Menschheit bereichere" (von Hentig 1996, 40). Der Zweck einer aufgeklärten, humanistischen Bildung ist also der Mensch, und zwar nicht seine Nutzbarmachung, sondern die Entfaltung seiner Selbst und die seiner Gattung.

Jedoch die „Logik des gesamtgesellschaftlichen Fortgangs [...] [folgt] nicht humanen Zwecken“, ,sondern der abstrakten Dynamik wirtschaftlicher Expansion. Das Strukturgesetz der modernen Gesellschaft, [..] hat das Tauschverhältnis von untereinander konkurrierenden Vertragspartnern zur Basis, die als Individuen nur im Hinblick auf ihre ökonomische Funktion in Betracht kommen" (Horkheimer \& Adorno 1975). Die Bildung des Menschen wird dadurch, nicht zuletzt im Zuge der Berufsbildung, für seine Verwertbarkeit instrumentalisiert. Und wenn sie folgerichtig als zweckrationalisiert betitelt werden kann, dann vor allem unter der Feststellung: Zweck und Sinn werden Eins und das, was daraus entsteht, ist der Primat ökonomischer Effektivität und die Schaffung von Arbeitskraft als konsequentem und immer präsenten Zweck beruflicher Bildung. Selbstentfaltung kommt in dieser Logik nur unter, wo sie in Produktivität übersetzbar ist. 
„Befähigung zur Bewältigung beruflicher Handlungssituationen“ und eine „Vorbereitung auf aktive Teilhabe und Teilnahme am Geschehen in Wirtschaft und Gesellschaft" (KMK 2009, 1) sind die politisch gefundenen Ziele der Berufsbildung. Zusätzlich haben alle einzelnen Stufen des Bildungssystems dafür Sorge zu tragen, dass die für den Übergang in das Berufsleben erforderlichen Kompetenzen vermittelt werden, damit den Anforderungen der gewerblichen Wirtschaft nachzukommen ist (NIHK 2016). Es zeigen sich bildungspolitische Positionen, welche die Interessen der Ökonomie mit den Zielen der Bildungsinstitutionen gleichsetzen und damit die Verwertungslogik selbst institutionalisieren.

Der beruflichen Bildung wird damit Integration zum Ziel gesetzt, und damit nach Adorno eine Verarbeitung des Subjekts, bei der durch die stattfindende Identifikation mit dem Beruf die eigene Identität in Gefahr schwebt, denn ,das allherrschende Identitätsprinzip, die abstrakte Vergleichbarkeit ihrer gesellschaftlichen Arbeit treibt“ die Subjekte, ,,bis zur Auslöschung ihrer Identität“ (Adorno 1997, B8, 13). Es kommt zum „Triumph der Integration“, bei dem sich die Individuen bis in ihr Innerstes mit der gesellschaftlichen Tauschlogik und den daraus abgeleiteten Glücksversprechungen, bezogen auf ihre eigene Lebensbestimmung, identifizieren würden (Adorno 1997, B8, 18). Das zur Lebenserhaltung notwendige Kapital, würde im zwanghaften Tausch gegen die eigene Freiheit zum Lebenselixier, nach dem fortwährend gestrebt werden muss (vgl. Schweppenhäuser 1996). Die Auszubildende würde in ihren ökonomischen Handlungen aufgehen. Wenn die Integration, die Anpassung, zum Bildungsideal ernannt wird, folgt die Prämisse, dass die Teilnahme am Reproduktionsgeschehen Sinn menschlichen Handelns ist. Emanzipation jedoch ist zuallererst Auflehnung.

Nach Marx vollzieht sich die Integration des Arbeiters in die kapitalistische Produktion als „Konsumtionsprozess der Arbeitskraft durch den Kapitalisten, “ dadurch „,verwandelt sich das Produkt des Arbeiters nicht nur fortwährend in Ware, sondern in Kapital, [in] Wert, der die wertschöpfende Kraft aussaugt" (vgl. Engels \& Marx 1968, 596). Der Mensch würde im Zuge der Integration in bestehende Produktionsprozesse zu einer Ware mit einem Tauschwert für die Ökonomie werden (ebd.). Und mehr bietet sich ihm nicht, denn der lebenslange (Fach-)Wissenserwerb ließe gedankliche Zusammenhänge mühselig erscheinen, wodurch sich reflexive Gedankengänge dem profitablen Qualifikationserwerb des Arbeitsmarktes unterordnen und die Selbstbesinnung des Geistes beeinträchtigt werden kann (Horkheimer \& Adorno 1975).

Und da, wo die Pädagogik Sinn vermitteln und als Erziehung zu Emanzipation und Mündigkeit Begleiter des Jugendlichen sein soll, dient sie nur mehr seiner Optimierung und seiner Verpflichtung für den Reproduktionsprozess kapitalistischer Verwertungsmechanismen. Als zukunftsfähig und selbstverantwortet gelten alleinig diejenigen, die im Raster des Kompetenzerwerbs ih- 
ren Arbeitsplatz auszufüllen vermögen. Wem die Pflichterfüllung zum höchsten Gut wird, bei dem war das Bildungssystem erfolgreich. Die Pädagogik, die sich diesem ihr entfremdeten Sinn nicht entzieht, sondern dadurch verschreibt, verweigert sich ihrer humanistischen Verantwortung. Erhebt sie vor der Arbeit und der Ökonomie den Zweck über den Menschen und die Verwertung zur Ratio, wird die Persönlichkeit, das Individuum, bald nicht mehr ihr Gegenstand, sondern ihr Mittel. Berufsbildung ist dann instrumentelle Bildung, zugeschnitten auf die zweckrationalisierte Vernunft der bürgerlichen Gesellschaft. Für den Auszubildenden hieße das Leistungs- und Selektionsdruck statt Selbstentfaltung und Erfahrung; es hieße Konkurrenzdenken statt Solidarität. ${ }^{6}$

\section{Anpassung als Auflösung des Spannungsfelds und Implikationen für die Berufspädagogik}

Abgebildet werden kann die Qualität des deutschen Berufsbildungssystems anhand der Konkurrenzfähigkeit der deutschen Wirtschaft auf dem Weltmarkt, die auf der Ausbildung hochqualifizierter Fachkräfte aufbaut und in den Bedingungen des dualen Berufsbildungssystems begründet ist (KMK 2010). Nicht der Einzelne, sich zum Individuum Entwickelnde, sondern der wirtschaftliche Erfolg des Landes gilt demzufolge als Indikator für eine qualitativ hochwertige Berufsausbildung. Anstatt seine Emanzipation zu forcieren, müsse die Einzelne ihren Erwerbsinteressen zum Wohle der Allgemeinheit unbedacht nachgehen (vgl. Schweppenhäuser 1996). Es entsteht ein Kreislauf der Bedürfnisbefriedigung, welche die Selbstverwirklichung ersetzt und bei dem die Interessen des Individuums nicht mehr von humanistischen Idealen, sondern von einer materiellen Identität und Profilierung geprägt sind. Zufriedenheit und gesellschaftliches Ansehen setzen sich fortan aus dem Erwerb von Konsumgütern zusammen und folgen damit nicht mehr der Autonomie und Authentizität emanzipatorischer Bildungsansätze (Schäfer 2015).

Der Wert des Menschen in der Logik der Verwertbarkeit ist also zuerst seine Arbeitskraft, die er als Leistung in den ökonomischen Schaffungsprozess einbringen kann, und zum zweiten seine Konsumkraft, über die er seine Arbeitskraft alimentiert. Wird dieser Sinnvereinnahmung das, dagegen fast schon

6 In dieser Logik konsequent antwortete auch ein Auszubildender in einem betrieblichen Modellversuch in den 1990er Jahren auf die Frage eines der Autoren, ob er denn bei der Optimierung eines vorbereitenden Lehrgang für seine nachfolgenden Auszubildenden in einer Lern- und Arbeitsinsel mitwirken wolle mit: „Nein! Dann werden die ja besser ausgebildet als ich und kriegen danach den Job!“ Eine willkommene Aussage, um den Widerspruch von Teamarbeit und Konkurrenz in der Produktion in dem betrieblichen Modellversuch zu thematisieren (Schapfel-Kaiser 2000). 
egoistisch erscheinende, Streben nach Selbstentfaltung als Überwindung gesellschaftlicher Heteronomie entgegengestellt, eröffnen sich für das Individuum im sich abzeichnenden Spannungsfeld drei mögliche Verfahrensgrundsätze. Erstens die Anpassung durch Überzeugung: Selbstverwirklichung wird zur Verwertungslogik umkodiert, sofern die Selbstwahrnehmung des Individuums nicht zu Unterdrückungserkenntnis, sondern zur Einsicht in die Richtigkeit jeder Teilhabe am Bestehenden führt - sei es aus Opportunismus, bspw. durch die Aussicht auf gesteigerte Partizipation an Konsumgütern und Statuszuwachs, oder aus dem Glauben an die Alternativlosigkeit zur kapitalistisch geordneten Gesellschaft. Zweitens die Anpassung aus der Notwendigkeit, vor der zwar ein Verständnis der Benachteiligung der eigenen Person im gesellschaftlichen Gefüge steht, aber gleichzeitig zur Vermeidung unvorteilhafter Konsequenzen, oder vielmehr aus Angst vor sozialer Ausgrenzung, die abverlangte Rolle eingenommen und weitest nötig ausgefüllt wird. Selbstverwirklichung wird zur Selbstverwertung willentlich und leidend gebogen; und drittens der Widerstand, der, auf der Ablehnung der gesellschaftlichen Verhältnisse beruhend, sich der Integration in diese verweigert und ihre Veränderung unterstützt. Selbstverwirklichung wird als Streben nach Emanzipation kategorisches Prinzip der Lebensgestaltung.

Wie Freud in seinen psychoanalytischen Arbeiten zeigte, und wie die Sozialisationsforschung bestätigt, wird das Subjekt entscheidend von seiner Umwelt, seiner es umgebenden gesellschaftlichen Realität, modifiziert. Das Spannungsfeld kann also für den Auszubildenden in der obsessiven Anpassung auflösbar erscheinen. Denn solange keine differenzierte Förderung der Emanzipation stattfindet, kann der Verblendungszusammenhang, der durch Manipulation und Integration entsteht, nicht überwunden werden (vgl. Schweppenhäuser 1996). Denn Kapital, das im Tausch gegen die eigene Freiheit generiert werden kann, wird benötigt, um die Lebenserhaltung zu ermöglichen (a. a. O., 145). Mit dem generierten Kapital kommen zum Prozess der Integration in Gesellschaft und Wirtschaft weitere, auf den Auszubildenden einwirkende Strukturen hinzu. Die Kultur- und Konsumgüterindustrie sorgt für individuelle Erlebnis- und Selbstverwirklichungsversprechen (vgl. Schäfer 2015), während neue Technologien auf jegliche Fragen eine Antwort zu haben scheinen (Lankau 2015). Unter dieser Beeinflussung klingt es nicht unwahrscheinlich, dass sich der Auszubildende mit Eintritt in die Arbeitswelt eine kapitalistische Persönlichkeit aufbaut, die ihn an einer kritischen Hinterfragung der bestehenden Verhältnisse hindert (vgl. Horkheimer 1980).

Und das ist im Grunde der Weg des geringsten Widerstands: die obsessive, sich ergebende Anpassung. Geleitet von der Triebnatur des Menschen werden die Auszubildenden durch die kapitalistisch-ökonomische Gesellschaftsstruktur so geformt, dass sich der integrative Prozess vollzieht. Da die Anpassung abverlangt wird, bevor ein kritisches Selbstverständnis dazu aufgebaut werden kann, scheint sich der Prozess unbewusst und schicksalsgleich zu vollziehen 
(vgl. Marcuse 1982; Schäfer 2015). Und so kommt es, wie Adorno feststellte, dass „Freiheit weithin Ideologie blieb; dass die Menschen ohnmächtig sind vom System und nicht vermögen, aus ihrer Vernunft ihr Leben und das des Ganzen zu bestimmen; ja dass sie nicht einmal mehr den Gedanken daran denken können, ohne zusätzlich zu leiden, bannt ihre Auflehnung in die verkehrte Gestalt; lieber wollen sie hämisch das Schlechtere denn den Schein des Besseren“ (Adorno 1997, B6, 109).

Es scheint also, als treibe die in der Verwertung mündende frühe Formung durch Gesellschaft, Bildung und Ökonomie den jugendlichen Auszubildenden dazu, Bestehendes zu akzeptieren. Das heißt jedoch nicht, dass Widerstand eine gänzlich abwegige Option ist. Denn die Auszubildende ist trotz der formulierten, manipulativen und in den gesellschaftlichen Strukturen implementierten Ökonomie, ein humanes Gattungswesen, das eigene Gefühle und Gedanken entwickeln kann. Demnach stellen Auszubildende als Erwerbsbürger*innen sowie „Waren- und Medienkonsumenten keine willenlosen Opfer von Ideologien“ dar, ,sondern handeln Kompromisse aus zwischen den eigenen Interessen und dem Medien-und Güterangebot" (Schweppenhäuser 1996, 165).

Es können letztlich als Prämissen, nicht nur einer kritisch-emanzipatorischen, sondern einer Berufspädagogik allgemein, abgeleitet werden: dass zum Ersten jegliche Auslassung solcher Entwicklung der Persönlichkeit, die nicht bloß dem beruflichen Kompetenzerwerb dient, die Anpassung an das Bestehende zur pädagogischen Prämisse korrumpiert, und zwar nicht nur zur Stabilisierung dessen, was ist, sondern auch zur Degeneration dessen, was über den Nutzen des Systemerhalts hinaus menschlich macht; und dass zum Zweiten auf Emanzipation gerichtete Bildung keine pädagogische Selbstverständlichkeit ist. Sie ist ein Schwimmen gegen den Strom in einer auf Unmündigkeit ausgerichteten Gesellschaft. Widerstand kann trotz oder gerade vor dem Anspruch der Selbstbefreiung nicht den Kräften des Einzelnen überlassen werden: Erziehung ist zwar selbst auch Zwang, aber dort legitimierbar, wo ihr Ziel die Befreiung davon ist.

\section{Anregungen aus Frankfurt und Aussicht}

Der vehementeste Anspruch der vorangegangenen Abhandlung ist es, wie auch der Wunsch ihrer Autoren, der kritisch-emanzipatorischen Bildungstheorie neue Aufmerksamkeit in der berufspädagogischen Wissenschaftsdisziplin zu verschaffen und ihr, als Folie der theoriegestützten Interpretation der gesellschaftlichen Institution Berufsbildung, neue Impulse zu setzen. Denn besonders zwei Aspekte ihrer Genese, deren Wurzeln bis zu Aufklärung und Marxismus zurückreichen, scheinen ihre Akzeptanz neben anderen Paradigmen 
der Berufsbildung zu gefährden: erstens, mit dem Blick auf die zurückliegende Blüte der Theorie, die Auffassung, dass zum Bezug und Nutzen der Prinzipien Emanzipation und Gesellschaftskritik für die Berufspädagogik bereits alles gesagt sei. Doch auch die größte Skeptikerin wird zugestehen, dass es zumindest wert sei, die scheinbare Wirkungslosigkeit des emanzipatorischen Ansatzes näher zu beleuchten und vor allem die verschiedenen Schriften und Argumentationslinien, die teilweise inkonsistent zueinanderstehen, systematisch aufzuarbeiten und auf das Gegenwärtige zu reflektieren, um ihren wissenschaftlichen Nutzen erneut zu beurteilen. Das mag den Anschluss an aktuelle bildungstheoretische Diskurse in der allgemeinen Erziehungswissenschaft ermöglichen, die auch dem Berufspädagogischen in der Bildung neue Beachtung erlauben können. Die zweite Gefährdung ist die Kollision mit dem die Berufsbildungswissenschaft derzeit prägenden, kritisch-rationalistischen Paradigma und des dort postulierten Kriteriums der Wertneutralität, welches für die Bildungswissenschaft nicht mehr als eine Illusion ist (vgl. Ketschau 2018). Auch wenn kein Grund erscheint, sich daran zu messen, muss sich jede kritischemanzipatorische pädagogische Arbeit, berechtigt vor ihrer Dogmatisierung und Politisierung, bewahren, wenn der Anspruch der Emanzipation nicht mehr nur als pädagogische Perspektive, sondern zur einzig wahren Bildung selbst verklärt würde. Wo Paradigma Ideologie würde, hätte Kritik als Prinzip und Ausgang des Widerspruchs keinen weiteren Platz.

Das Erörterte bietet Raum für anschließende Fragestellungen und Untersuchungen, die auch über die Möglichkeiten theoretisch-philosophischer Reflexion hinausgehen. Das dargestellte Spannungsfeld lässt sich in eine Makround Mikroebene zerlegen, wobei der vorliegende Beitrag für die Untersuchung der Makroebene Hinweise liefert. Auf einer Mikroebene bliebe zu untersuchen, wie sich das Spannungsfeld für Auszubildende und Beschäftigte in ihrer betrieblichen Realität manifestiert, ob und wie sie es wahrnehmen, und wie sie damit eventuell umgehen. Doch nicht nur die Position der Lernenden erscheint hier interessant, auch die der Lehrenden in Schule und Betrieb. Von ihrer Warte wird die Herausforderung der kritischen Berufsbildungstheorie ausgerichtet auf die Befähigung der Auszubildenden zum Lesen der „Textur“ ihres jeweiligen beruflichen Kontextes, wie Matti Vesa Volanen anführt: „The general element of vocational Bildung is thus bound up with the materiality of social texture, that is, with a reflection of dead work. This Allgemein is, then, never merely symbols of or texts by a productive mind, but an unfolding of the hieroglyph of real work, already completed." (Vesa Volanen 1999, 173). Die Untersuchung, wie Betrieb und Schule zunehmend gelingt, stände immer vor dem Anspruch, dass eine emanzipative Wiederbesinnung der Berufspädagogik, die auf eine Überwindung des Leidens abzielt, sich den Ursachen dieses Leidens grundlegend stellen muss, ohne, im angenehmen Raum des Philosophischen verweilend, die dogmatische Verkürzung zu riskieren. 
Genauso wie die empirische Konkretisierung scheint die begriffliche Anreicherung der kritisch-emanzipatorischen Berufsbildungstheorie ertragreich. Sie kann den Fortschritt der Überwindung von Ungleichheit, beispielsweise im Hinblick auf Gender oder Migrationshintergrund, als hoffnungsschürenden Impuls aufnehmen. Damit öffnet sie sich deutlich der Frage der sozialen Gerechtigkeit, die auch im gesellschaftlichen Diskurs an Relevanz zu gewinnen scheint (Bylinski \& Rützel 2011). Neben neuen Begriffen sind aber auch ihre Grundbegriffe und Prämissen zu rekonstruieren und zu erweitern, begonnen bei der Emanzipation und ihrem Verhältnis zu Beruf, Arbeit und Berufsbildung. Denn wie weit ist sie vor dem oder gerade durch den Beruf denkbar, wo zeichnen sich ihre Widersprüche als Erziehungsideal und wie weit ist sie für Bildung und Erziehung als Utopie überhaupt erträglich? Was ist emanzipativer Widerstand und was destruktiver? Gilt es latenten Widerstand genauso zu würdigen wie kollektiven, offensiven Widerstand? Wo wird Gestaltungsfähigkeit zu einer echten Opposition, um die Welt zum Besseren zu verändern?

All das verlangt nach Erkundungen in der betrieblichen und berufsschulischen Lebenswelt und einer kategorialen Fundierung, die an die Sozialisationsstudien der 1970-1990er Jahre anknüpfen können und sich des zwischenzeitlich erfolgten forschungsmethodologischen Fortschritts bedienen. Kategorien wären zunächst theoretisch zu begründen und dann im Diskurs mit den Subjekten in der beruflichen Bildung und den Befunden zu überarbeiten und ggf. weiter zu entwickeln. Dabei kann, so unsere Zuversicht, Forschung und der mit ihr verbundene Diskurs zur Aufklärung und Überwindung vermeidbarer Herrschaft beitragen, wenn sie offen gegenüber der Lebenswelt und im gesellschaftlichen Diskurs erfolgt. „Der Fortschritt der Emanzipation ergibt sich aus dem Zusammenwirken vieler theoretischer und praktischer Aktivitäten. Ihr Weg ist schwierig, ihr Erfolg ungewiß, ihre Theorie darum nicht dogmatisch, sondern hypothetisch, ihr Vorgehen experimentell, ihr Prüfstein ist die kommunikative Erfahrung nachlassenden Leidens“ (Lempert 1974, 36).

\section{Literatur}

Adorno, T.-W. (1997). Negative Dialektik. Jargon der Eigentlichkeit. Zur deutschen Ideologie. In R. Tiedemann (Hrsg.), Gesammelte Schriften in zwanzig Bänden. Band 6. Frankfurt am Main: Suhrkamp.

Adorno, T.-W. (1997). Soziologische Schriften. In R. Tiedemann (Hrsg.), Gesammelte Schriften in zwanzig Bänden. Band 8. Frankfurt am Main: Suhrkamp.

Allais-Matseleng, S. (2017). Labour market outcomes of NQF. What is known about what they can and can't do? In F. Kaiser \& S. Krugmann (eds.), Social Dimension and Participation in Vocational Education and Training, (pp. 4-15). Rostock: University. 
Beck, U., Brater, M. \& Daheim, H. (Hrsg.). (1980). Soziologie der Arbeit und der Berufe. Grundlagen, Problemfelder, Forschungsergebnisse. Reinbek bei Hamburg: Rowohlt.

Blankertz, H. (1983). Berufsausbildung als Prüfstein für die pädagogische Qualität des öffentlichen Unterrichtswesens. Zeitschrift für Berufs- und Wirtschaftspädagogik, H.11, 803-810.

Büchter, K. (2017). Allgemeinbildung und Berufsbildung - übergreifende Widersprüche historisch betrachtet. In P. Schlögl, M. Stock, D. Moser, K. Schmit \& F. Gramlinger (Hrsg.), Berufsbildung, eine Renaissance? (S. 21-43). Bielefeld: WBV.

Büchter, K. \& Kipp, M. (2009). Berufsbildung in der Zeit der Reformpädagogik - 18901933. In A. Diettrich, D. Frommberger \& J. Klusmeyer (Hrsg.), Akzentsetzungen in der Berufs- und Wirtschaftspädagogik, Holger Reinisch zum 60sten Geburtstag. Online: http://www.bwpat.de/profil2/buechter_kipp_profil2.shtml (20.11.2018).

Büchter, K. \& Kipp, M. (2014). Von der Lehrlingsbewegung zur „Befähigung zur Mitgestaltung“ - (ent-)politisierte Jugend als Leitidee der Lehrerbildung? In bwp@ Berufs- und Wirtschaftspädagogik - online, Profil 3, 1-23. Online: http://www. bwpat.de/profil3/buechter_kipp_profil3.pdf (22.8.2018).

Bylinski, U. \& Rützel, J. (2011). „Ausbildung für alle“ braucht eine Pädagogik der Vielfalt. $B W P, 2 / 2011,14-17$.

Dammer, K.-H. (Hrsg.). (2015). Vermessene Bildungsforschung: wissenschaftsgeschichtliche Hintergründe zu einem neoliberalen Herrschaftsinstrument. Baltmannsweiler: Schneider Verlag Hohengehren.

Dobmeier, F. (2018). Bildung der Angst. Reflexionen zur Verhinderung potenzieller Bildungsprozesse durch psychopolitische Machttechniken gouvermentaler Angstdesigns. Manuskript des Vortrags auf dem DGFE-Kongress 2018.

Engels, F. \& Marx, K. (1968). Das Kapital. Bd. I. Abschnitt 7. Berlin/DDR: Dietz.

Geißler, K. A. (Hrsg.). (1974). Berufserziehung und kritische Kompetenz. München: Reinhardt.

Graeber, D. (Hrsg.). (2018): Bullshit-Jobs. Vom wahren Sinn der Arbeit. Stuttgart: Klett-Cotta.

Hentig, H. von (Hrsg.). (1996). Bildung. München, Wien: Hanser.

Heydorn, H.-J. (Hrsg.). (1970). Über den Widerspruch von Bildung und Herrschaft. Frankfurt: Europäische Verlagsanstalt.

Heydorn, H.-J. (1971). Jan Amos Comenius: Grundriß eines Vermächtnisses. In H.-J. Heydorn (Hrsg.), Zur bürgerlichen Bildung. Anspruch und Wirklichkeit. Bildungstheoretische Schriften, Band 1, (S. 297-202). Frankfurt: Glashütten.

Horkheimer, M. \& Adorno, T.-W. (Hrsg.). (1975). Dialektik der Aufklärung - Philosophische Fragmente. Frankfurt am Main: Fischer Taschenbuch Verlag.

Kaiser, F. (2016). Berufliche Bildung und Emanzipation. Heydorns Impulse für eine kritische Berufsbildungstheorie sowie Stolpersteine aus eigener berufspädagogischer Sicht. In F. Ragutt \& F. Kaiser (Hrsg.), Menschlichkeit der Bildung, (S. 181-197). Paderborn: Schöningh.

Kaiser, F. (2017). Sinn und Charakteristik kaufmännischer Tätigkeit und die Widersprüche der Bildung von kaufmännischen Angestellten in einer Demokratie. Analysen und Implikationen. In T. Tramm, T. Schlömer \& C. Thole (Hrsg.), bwp@ Spezial 14: Homo oeconomicus oder Ehrbarer Kaufmann - Reflexionen zum Verhältnis der Wirtschaftspädagogik zu den Wirtschaftswissenschaften. Online: http://www.bwpat.de/spezial14/kaiser_bwpat_spezial14.pdf (16.11.2017). 
Kaiser, F. (2019). Studierende zur kritischen Gestaltung der beruflichen Bildung ermutigen: Zur zentralen Aufgabe berufspädagogischer Lehramtsstudiengänge in einer demokratischen Gesellschaft. In C. Kalisch \& F. Kaiser (Hrsg.), Bildung beruflicher Lehrkräfte. Wege in die pädagogische Königsklasse, (S. 33-46). Gütersloh: W. Bertelsmann.

Kehren, Y. (Hrsg.). (2016). Bildung für nachhaltige Entwicklung. Zur Kritik eines pädagogischen Programms. Baltmannsweiler: Schneider Verlag Hohengehren.

Ketschau, T.J. (2018). Reflexionen zur Philosophie der Berufsbildung. Rekonstruktion und Perspektiven. Zeitschrift für Berufs- und Wirtschaftspädagogik, 114(1), 85108.

Kipp, M. (1992). Mit Kopf, Herz und Hand? - Kritische Anmerkungen zu ,ganzheitlichen" Qualifizierungskonzepten. In G. Pätzold (Hrsg.), Handlungsorientierung in der beruflichen Bildung, (S. 123-138). Frankfurt: GAFB.

KMK. (2010). Beschluss der Kultusministerkonferenz. Erklärung der Kultusministerkonferenz für eine zukunftsorientierte Gestaltung der dualen Berufsausbildung. München: Luchterhand.

KMK. (2009). Beschluss der Kultusministerkonferenz. Erklärung der Kultusministerkonferenz zur zukünftigen Stellung der Berufsschule in der dualen Berufsausbildung. München: Luchterhand.

Koneffke, G. (1982). Zur Erforschung der Industrieschule des 17. Und 18.Jahrhunderts. In G. Koneffke (Hrsg.), (2018): Widersprüche bürgerlicher Mündigkeit. Materialistische Bildungstheorie als politische Explikation der Pädagogik, (S. 7-30). Bd. II. Hohengehren: Schneider.

Koneffke, G. (1987). Widersprüche im frühbürgerlichen Bildungsbegriff. In R. Drechsel (Hrsg.), Ende der Aufklärung? Zur Aktualität einer Theorie der Bildung, (S. 131-148). Bremen: Universität.

Koneffke, G. (1995). Bildung und Herrschaft. Überlegungen zur Bildungsreform vor der Jahrtausendwende. In P. Euler \& L. Pongratz (Hrsg.), Kritische Bildungstheorie - zur Aktualität Heinz-Joachim Heydorns, (S. 39-58). Weinheim: DSV.

Kutscha, G. (2008). Bildung im Medium des Berufs? Abschlussvorlesung. Unveröffentlichtes Manuskript. Online: https:/www.uni-due.de/imperia/md/content/berufspaedagogik/kutscha/kutscha abschlussvorlesung_nov2008.pdf (21.11.2018).

Lankau, R. (2015). Fragen Sie Alexa. Die Entmündigung des Individuums durch die Vermessung der Welt. In K.-H. Dammer, T. Vogel \& H. Wehr (Hrsg.), Zur Aktualität der kritischen Theorie für die Pädagogik, (S. 277-297). Wiesbaden: Springer Fachmedien.

Lempert, W. (Hrsg.). (1971). Leistungsprinzip und Emanzipation. Studien zur Realität und Reform des beruflichen Bildungswesens. Frankfurt: Suhrkamp.

Lempert, W. (Hrsg.). (1974). Berufliche Bildung als Beitrag zur gesellschaftlichen Demokratisierung. Vorstudien für eine politisch reflektierte Berufspädagogik. Frankfurt: Suhrkamp.

Lempert, W. (2008). Zwei einfältige Fragen an alle 'gelernten' Berufs- und Wirtschaftspädagogen: wider die Unvernunft derzeit gravierender, weltweit grassierender, längst dominierender, noch eskalierender, weiter sich steigernder ökonomistischer Perversionen menschlichen Handelns, Lehrens und Lernens. Zeitschrift für Berufs- und Wirtschaftspädagogik, 104(3), 467-468.

Lisop, I. \& Huisinga, R. (Hrsg.). (2004). Arbeitsorientierte Exemplarik. Subjektbildung - Kompetenz - Professionalität. Ein Lehrbuch. Frankfurt: G.A.F.B. 
Mayer, E., Schumm, W., Flaake, K, Gerberding, H \& Reuling, J. (Hrsg.). (1981). Betriebliche Ausbildung und gesellschaftliches Bewußtsein. Die berufliche Sozialisation Jugendlicher. Frankfurt, New York: Campus.

Milev, J. (2013). Design(-Governance). In L. Koch (Hrsg.). Angst. Ein interdisziplinäres Handbuch, (S. 226-235). Stuttgart: Metzler.

Müllges, U. (Hrsg.). (1967). Bildung und Berufsbildung. Die theoretische Grundlegung des Berufserziehungsproblems durch Kerschensteiner, Spranger, Fischer und Litt. Ratingen: Henn.

Nell-Breuning, O. (1975). Können Neoliberalismus und katholische Soziallehre sich verständigen? In H. Sauermann \& E.-J. Mestmäcker (Hrsg.), Wirtschaftsordnung und Staatsverfassung (Festschrift Franz Böhm zum 80. Geburtstag), (S. 459-470). Tübingen: JCB Mohr.

Niedersächsische Industrie- und Handelskammer (NIHK). (2016). Bildungspolitische Positionen. Hannover.

Pfeiffer, S. (2014). Digital Labour and the Use-value of Human Work. On the Importance of Labouring Capacity for understanding Digital Capitalism. tripleC. Journal for a global sustainable Information Society, 12(2), 599-619, Online: https://www.triple-c.at/index.php/tripleC/article/view/545/611 (20.08.2017).

Pongratz, L. A. (1995). Aufklärung und Widerstand. Kritische Bildungstheorie bei Heinz-Joachim Heydorn. In P. Euler \& L. Pongratz (Hrsg.), Kritische Bildungstheorie - zur Aktualität Heinz-Joachim Heydorns, (S. 11-38). Weinheim: DSV.

Rebmann, K., Schlömer, T., Feldkamp, D., Jahncke, H. \& Lüllau, C. (2017). The O1denburg Model of Vocational Education and Training for Sustainable Development. Design within the Model Test "Advanced Training to Become a Renewable Energies/Energy Efficiency Specialist (German Chamber of Crafts)". Journal of Sustainable Development, 10(3), 1-13.

Reichenbach, R. (2009). Bildung und Autorität. In C. Bünger, R. Mayer, A. Messerschmidt \& O. Zitzelsberger (Hrsg.), Bildung der Kontrollgesellschaft. Analyse und Kritik pädagogischer Vereinnahmungen. Festschrift für L. A. Pongratz, (S. 7184). Paderborn: Schöningh.

Schäfer, A. (2015). (Halb-)Bildungsräume. Zwischen Kulturkritik und „,modernem Hedonismus“. In K.-H. Dammer \& T. Vogel, H. Wehr (Hrsg.), Zur Aktualität der kritischen Theorie für die Pädagogik, (S. 109-125). Wiesbaden: Springer.

Schapfel-Kaiser, F. (1998). Vom Treibholz zum Floß. Lernen an und mit der Biographie in der beruflichen Bildung. Ein Aspekt kritisch-subjektorientierter Berufspädagogik. In J. Rützel \& W. Sesink (Hrsg.), Jahrbuch für Pädagogik 1998 „, Bildung nach dem Zeitalter der großen Industrie“, (S.127-147). Frankfurt: Lang.

Schapfel-Kaiser, F. (2000). Die Gleichzeitigkeit von Kooperation und Konkurrenz Erfahrungen mit Gruppenarbeit und Qualitätssicherung im Modellversuch FLAI. In R. Schlausch \& M. Sander (Hrsg.), Herausforderungen an die Gestaltung der metalltechnischen Berufsausbildung, (S. 28-46). Neusäß: Kieser.

Schapfel-Kaiser, F. (2003). Kritisch-subjektorientierte Berufsbildungstheorie und exemplarische Praxisfelder. In A. Bernhard, A. Kremer \& M. Rießland (Hrsg.), Kritische Erziehungswissenschaft und Bildungsreform, Bd. 2, (S. 56-75). Hohengehren: Schneider.

Schweppenhäuser, G. (Hrsg.). (1996). Theodor W. Adorno zur Einführung. Hamburg: Junius. 
Stütz, G. (Hrsg.). (1970). Berufspädagogik unter ideologiekritischem Aspekt. Frankfurt: Suhrkamp.

Vesa Volanen, M. (1999). Bildung through vocational education. In J. Lasonen (ed.), Workforce preparation in a global context. Occasional papers 8, (pp.167-177). Jyväskylä: Institute for Educational Research.

Welskopp, T. (2017). Kapitalismus als Landnahme. Von einem Theoriestrang von Karl Marx bis Rosa Luxemburg. Mittelweg 36, „, Theorien des Kapitalismus, Heft 6/2017, 19-28.

Ziegler, J. \& Gebauer, T. (2018). Wir sind nicht ohnmächtig. Eine Debatte über eine gelähmte UN, neoliberalen Wahn und radikalen Zorn. Medico international Jubiläumsrundschreiben, 1/2018, 10-14. 



\title{
Entwicklung eines Reifegradmodells der Digitalisierung für Bildungsorganisationen
}

\author{
Marc Egloffstein, Tobias Heilig und Dirk Ifenthaler
}

\section{Problemstellung}

Die zunehmende Durchdringung vielfältiger Lebensbereiche durch digitale Technologien führt zu tiefgreifenden Veränderungen für das Lernen und Arbeiten und berührt die Berufliche Bildung im Kern. Drei maßgebliche Funktionen von digitalen Technologien für das berufliche Lehren und Lernen lassen sich dabei abgrenzen: (1) Als Lerninstrumente bieten digitale Technologien neue Optionen für die methodische Gestaltung von Lernprozessen. (2) Als Arbeitsinstrumente verändern sie berufliche Arbeits- und Geschäftsprozesse. (3) Als Universalinstrumente beeinflussen sie den Alltag und damit die Lernvoraussetzungen der Beteiligten (Euler \& Wilbers 2018). Bildungsorganisationen stehen vor der Herausforderung, den daraus erwachsenden Aufgaben mit passenden Maßnahmen der Personal- (PE) und Organisationsentwicklung (OE) zu begegnen. Dabei geht es nicht nur darum, Aspekte einer „Berufsbildung 4.0“ (Wilbers 2016) über kurz oder lang in der Aus- und Weiterbildungspraxis zu verankern (vgl. Gerholz \& Dormann 2017). Ziel ist es vielmehr, den weitreichenden Veränderungen durch die digitale Transformation im Großen wie im Kleinen adäquat begegnen zu können. Den inhaltlichen Bezugspunkt für entsprechende PE-Maßnahmen stellen Medienkompetenzmodelle dar (z.B. Härtel et al. 2018; Seufert, Guggemos, Tarantini \& Schumann 2019). Ein vergleichbarer Bezugspunkt für OE-Maßnahmen in Bildungsorganisationen fehlt indes.

Hier setzt der vorliegende Beitrag an. Die übergreifenden Fragestellungen lauten: Wie kann ein Reifegradmodell aussehen, das als Bezugsbasis für die Begleitung und Gestaltung von Digitalisierungsprozessen in Bildungsorganisationen dienen kann? Mit welchen Indikatoren kann die , digitale Reife ‘ einer Bildungsorganisation beschrieben werden, und welche Ausprägungen dieser Indikatoren treten dabei in der Praxis auf? Nach der Vorstellung des zu Grunde liegenden Reifegradansatzes wird ein Modell der ,digitalen Reife' von Bildungsorganisationen skizziert. Daran anknüpfend wird die Operationalisierung und Anwendung des Modells im Projekt \#ko.vernetzt beschrieben. 


\section{Reifegradmodelle und Reifegradindizes}

Die Wurzeln von Reifegradmodellen (engl. maturity models) liegen in den 1970er Jahren (Lahrmann \& Marx 2010). Disziplinär sind Reifegradmodelle aktuell vor allem in der Wirtschaftsinformatik zu verorten (Wendler 2012). Reife bezeichnet dabei ,eine evolutionäre Verbesserung hin zu einem Zielzustand oder zu einem natürlichen Endzustand“ (Marx, Wortmann \& Mayer 2012, 190). Reife kann sich auf Kompetenzen, Fähigkeiten, Geschäftsprozesse oder Produkte beziehen (de Bruin, Freeze, Kaulkarni \& Rosemann 2005). Die Entwicklung zu einem höheren, fortschrittlicheren Stadium erfolgt stufenweise und wird durch Reifegrade beschrieben (Becker, Knackstedt \& Pöppelbuß 2009; Egeli 2016).

Reifegradmodelle setzen sich im Wesentlichen aus vier Bestandteilen zusammen (Christiansen \& Gausemeier 2010; Egeli 2016): Die Reifegrade entsprechen den verschiedenen Reifestufen, die den Leistungsstand einer Organisation abbilden. Die Dimensionen kategorisieren den Untersuchungsbereich (Christiansen \& Gausemeier 2010). Jede Dimension wird durch mehrere Indikatoren, Eigenschaften, Aktivitäten oder Maßnahmen beschrieben (Lahrmann \& Marx 2010). Beim Reifeprinzip lassen sich zwei grundlegende Vorgehensweisen unterschieden: Bei einem Stufenmodell wird die nächsthöhere Stufe erst dann erreicht, wenn alle Elemente des vorherigen Levels erfüllt sind (Egeli 2016). Bei kontinuierlichen Reifegradmodellen dürfen sich die jeweiligen Ausprägungen der Dimensionen auf verschiedenen Levels befinden. Die Leistungsbewertung ermittelt Ausgangszustände der betrachteten Organisationen (Christiansen \& Gausemeier 2010) und ordnet diese auf den Reifestufen ein. Als Beurteilungsinstrumente kommen bspw. qualitative Beschreibungen oder quantitative Erfassungen in Frage (Lahrmann \& Marx 2010).

Die Entwicklung von Reifegradmodellen umfasst fünf idealtypische Phasen: Die erste Phase ist die Problemdefinition. Hierbei werden Zielsetzung, Forschungsbereich und Zielgruppen festgelegt (Becker et al. 2009). AnschlieBend erfolgt ein Vergleich bestehender Reifegradmodelle. Daran anknüpfend wird die Entwicklungsstrategie festgelegt. Optionen sind: vollständige Neuentwicklung sowie Weiterentwicklung, Kombination oder Rekonfiguration bestehender Modelle. Die Reifegradmodellierung (Ausarbeitung der Dimensionen und Stufen, bottom-up vs. top-down) erfolgt iterativ. In der Phase der Modellevaluation wird untersucht, ob das Modell einen Beitrag zur Lösung der anfänglichen Problemstellung leistet (Becker et al. 2009).

Reifegradindizes sind das Ergebnis der Operationalisierung von Reifegradmodellen und verdichten die Modelldimensionen zu wenigen Kennzahlen, mitunter gar zu einem einzigen Wert. Dadurch soll eine gute Handhabbarkeit für Beschreibungs-, Erklärungs- und/oder Prognosezwecke erzielt werden. 


\section{Modellierung der ,digitalen Reife' von Bildungs- organisationen}

\subsection{Reifegradmodelle der Digitalisierung}

Das Konstrukt der ,digitalen Reife" beschreibt den Status Quo der digitalen Transformation einer Organisation (Chanias \& Hess 2016). ,Digitale Reife wird in der Regel kontextbezogen operationalisiert, was zu einer Vielzahl von unterschiedlichen Modellen im Unternehmenskontext führt (Remane, Hanelt, Wiesboeck \& Kolbe 2017). Reifegradmodelle mit Bildungsbezug finden sich vor allem für IT-bezogene Fragestellungen im Hochschulkontext, hier allerdings fast durchweg noch in einem frühen Stadium der Entwicklung (Carvalho, Pereira \& Rocha 2019). Referenzmodelle für Bildungsorganisationen insbesondere der Beruflichen Bildung sind dagegen kaum zu finden. Für eine Modellierung in diesem Kontext bietet sich demnach eine Weiterentwicklung, Rekonfiguration oder Kombination bestehender Modelle mit Unternehmensbezug an.

Abbildung 1 zeigt exemplarische Reifegradmodelle der Digitalisierung im Überblick. Diese Referenzmodelle beziehen sich alle auf einen Organisationskontext, weisen unterschiedliche Dimensionen (und damit verbundene Indikatoren) sowie mehrere Reifestufen auf. Hinsichtlich der Inhalte der unterschiedlichen Dimensionen bestehen materiell nur wenige Unterschiede, was auf eine übergreifende Vorstellung von, digitaler Reife' schließen lässt - auch über unterschiedliche Zielstellungen und Zielgruppen hinweg. 
Abb. 1: Referenzmodelle, digitaler Reife' im Überblick

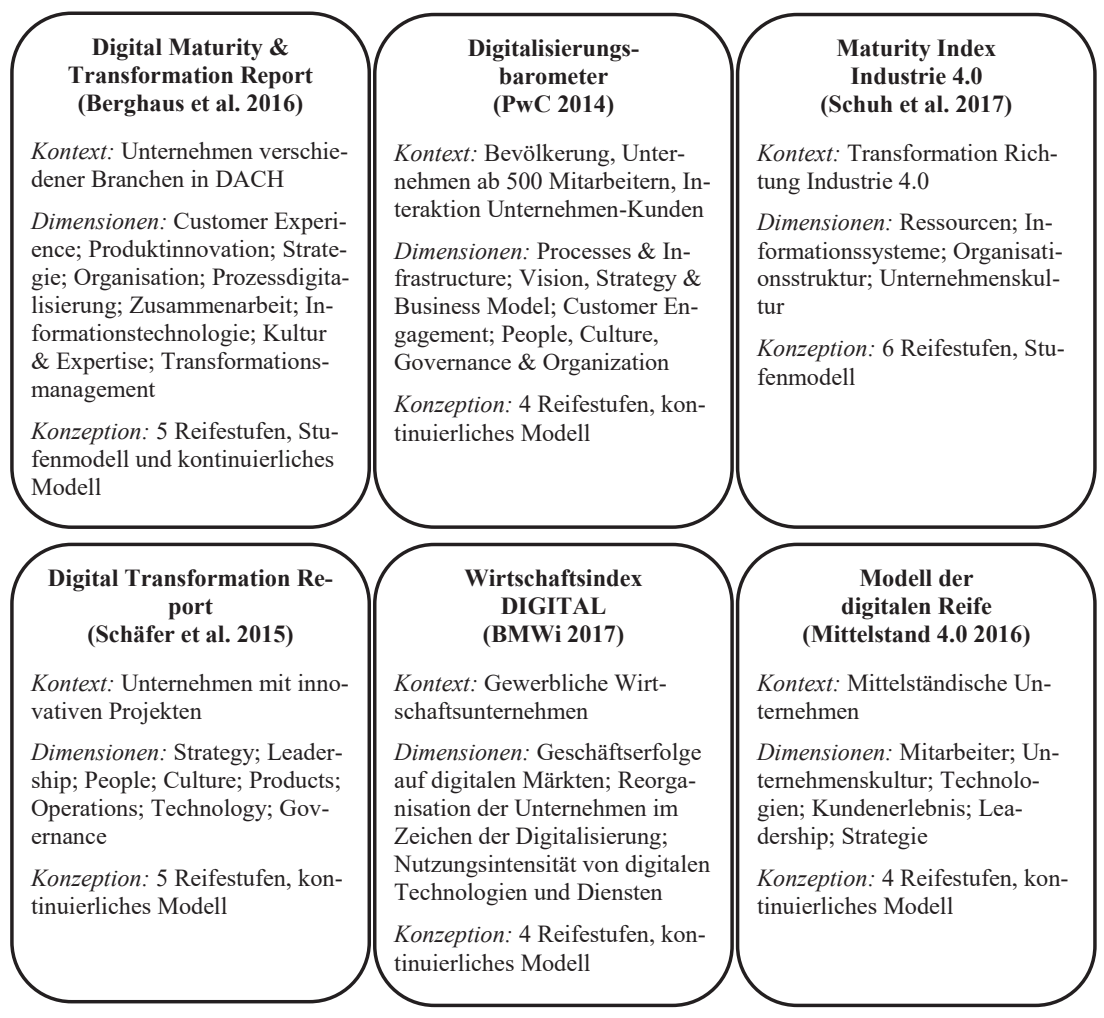

Quelle: eigene Darstellung

\subsection{Dimensionen des digitalen Reifegrads von Bildungsorganisationen}

In For-Profit-Bildungsorganisationen werden neben erwerbswirtschaftlichen Zielen zusätzliche pädagogische Zielstellungen verfolgt. Bestehende Modelle zum digitalen Reifegrad müssen daher um pädagogisch akzentuierte Modellkomponenten erweitert werden. Aus der Synopse und Anpassung der Referenzmodelle ergeben sich zunächst fünf Dimensionen und Indikatoren (Tabelle 1). Die sechste Dimension ,Digitales Lehren und Lernen' bezieht sich auf die Kernprozesse einer jeden Bildungsorganisation und wird deshalb explizit samt entsprechender Beispielindikatoren in die Modellierung aufgenommen. 
Tab.1: Dimensionen des Reifegradmodells für Bildungsorganisationen

\begin{tabular}{|c|c|}
\hline Dimension & Beispiel-Indikatoren/Inhalte \\
\hline $\begin{array}{l}\text { Ausstattung und } \\
\text { Technik }\end{array}$ & $\begin{array}{ll}\text { - } & \text { Ausstattung mit digitalen Geräten, Software } \\
\text { - } & \text { Aktualität der Infrastruktur } \\
& \text { Einheitliche Technik, Standards }\end{array}$ \\
\hline $\begin{array}{l}\text { Strategie und } \\
\text { Führung }\end{array}$ & $\begin{array}{ll}\text { - } & \text { Existenz und Umsetzung einer digitalen Strategie } \\
\text { - } & \text { Führungskräfte treiben Digitalisierung priorisiert voran } \\
\text { - } & \text { Evaluation von neuen Technologien } \\
& \text { Demokratischer Führungsstil, Gewährung von } \\
& \text { Gestaltungsfreiräumen }\end{array}$ \\
\hline Organisation & $\begin{array}{ll}\text { - } & \text { Ausreichende finanzielle Ressourcen } \\
\text { - } & \text { Technischer Support (intern vs. externe Dienstleister) } \\
\text { - } & \text { Pädrale Beschaffung und Wartung } \\
\text { Pägische Unterstützung }\end{array}$ \\
\hline Mitarbeitende & $\begin{array}{ll}\text { - } & \text { Wissen/Fähigkeiten im Umgang mit digitalen Technologien } \\
\text { - } & \text { Nutzung von Geräten und Diensten } \\
\text { - } & \text { Einstellungen } \\
& \text { Weiterbildungsbereitschaft }\end{array}$ \\
\hline Kultur & $\begin{array}{ll}\text { - } & \text { Offenheit für neue Technologien } \\
\text { - } & \text { Bereitschaft für Veränderungen } \\
\text { offene Kommunikation, gegenseitige Unterstützung }\end{array}$ \\
\hline $\begin{array}{l}\text { Digitales Lehren } \\
\text { und Lernen }\end{array}$ & $\begin{array}{ll}\text { - } & \text { Digitale Plattformen, e-Learning-Angebote } \\
\text { - } & \text { Arbeit mit Tablets/digitalen Geräten im Unterricht } \\
\text { - } & \text { Digitale Bildung als Unterrichtsziel } \\
& \text { Nutzung von Learning Analytics } \\
\end{array}$ \\
\hline
\end{tabular}

Quelle: eigene Darstellung.

Die Dimensionen sind nicht isoliert voneinander zu betrachten, sondern beeinflussen sich gegenseitig. Um die Indexwerte der digitalen Reife einordnen zu können, wurden in Anlehnung an die Referenzmodellierungen fünf Reifegrade festgelegt. Den Ausgangspunkt der Setzung bildet der Reifegrad 3, welcher einer mittleren Reifestufe entspricht und inhaltlich für eine bereits grundlegend umgesetzte Digitalisierung steht. Zur weiteren Differenzierung werden in Anlehnung an die Referenzmodelle jeweils zwei weitere Reifestufen darüber und darunter definiert. Die Konzeption folgt dabei dem kontinuierlichen Modellansatz. 


\section{Operationalisierung des digitalen Reifegrads von Bildungsorganisationen im Projekt \#ko.vernetzt}

\subsection{Hintergrund und Forschungsfragen}

\#ko.vernetz ${ }^{1}$ ist ein Forschungs- und Entwicklungsprojekt, das individuelle Medienkompetenzen sowie Systeme, Strukturen und Prozesse in Bildungsorganisationen in den Blick nimmt und damit, digitale' $\mathrm{PE}$ und $\mathrm{OE}$ kombiniert (Helbig, Egloffstein, Hofhues \& Ifenthaler in Vorb.). Verbundpartner*innen sind das Institut Jugend Film Fernsehen Berlin-Brandenburg e.V., die Universität zu Köln, die Universität Mannheim sowie, als Praxispartnerin, die Kolping-Bildungswerk Paderborn gGmbH. Die Geschäftsfelder des Kolping Bildungswerkes (KBW) decken einen weiten Teil der Bildungskette ab. Dabei bedingen heterogene und ambivalente Zugänge zu digitalen Technologien ein uneinheitliches Verständnis von Digitalisierungsprozessen und divergierende Anforderungen an medienbezogene Bildungsarbeit. Das KBW steht somit exemplarisch für die vielfältigen Herausforderungen, mit denen Bildungsträger durch die Digitalisierung konfrontiert werden. Am Beispiel des KBW werden in der vorliegenden Studie folgende Forschungsfragen beantwortet:

- FF 1: Wie stellt sich die Nutzung digitaler Medien und Technologien im betrachteten Kontext dar, und gibt es dabei Unterschiede zwischen beruflicher und privater Nutzung?

- FF 2: Wie stellt sich die Einstellung gegenüber Aspekten der Digitalisierung im betrachteten Kontext dar?

- FF 3: Wie stellt sich der Index der digitalen Reife im betrachteten Kontext dar, und gibt es Unterschiede zwischen verschiedenen Personengruppen?

\subsection{Methode und Stichprobe}

Zur Erfassung von Aspekten der Digitalisierung im KBW wurde ausgehend von vorliegenden Instrumenten (z. B. Initiative D21 2018) ein sogenanntes ,Digitalisierungsbarometer ${ }^{6}$ entwickelt. Dieser nicht-personalisierte Fragebogen besteht aus 49 Items zur Digitalisierung, die im Wesentlichen über sechsstufige Likert-Skalen beantwortet werden, sowie aus acht Items zum soziodemographischen und beruflichen Hintergrund. Tabelle 2 zeigt die Inhaltsbereiche des Instruments.

\footnotetext{
${ }^{1}$ Gefördert aus Mitteln des Bundesministeriums für Bildung und Forschung (BMBF) und des Europäischen Sozialfonds (ESF) (Förderkennzeichen 01PZ16002A-D).
} 
Tab. 2: Inhaltsbereiche des Digitalisierungsbarometers

\begin{tabular}{ll} 
Bereich & Items \\
\hline Allgemeine Einschätzung & 1 \\
Vorstellungen von Digitalisierung & 8 \\
Nutzung digitaler Medien & 20 \\
Bewertung digitaler Medien & 5 \\
Digitalisierung im Arbeitskontext & 7 \\
Einstellung zur Digitalisierung & 6 \\
\hline
\end{tabular}

Quelle: eigene Darstellung.

Zur Berechnung des Reifegrad-Index erfolgte eine theoriegeleitete Zuordnung der Items zu den Dimensionen des Reifegradmodells. Eine faktoren- und reliabilitätsanalytische Überprüfung dieser Zuordnung führte zum Ausschluss zweier Items. Die Antworten der Likert-Skalen wurden in ein Punktesystem überführt, sodass für jede der sechs Modelldimensionen ein normierter Teilindex berechnet werden konnte. In die Berechnung des Gesamtindex floss die Dimension der Mitarbeitenden mit dreifacher Gewichtung ein, was der Anzahl der Items und der Konzeption der Mitarbeiter*innen-Befragung Rechnung trägt. Den fünf Reifestufen wurden dabei folgende Punktzahlen im Gesamtindex zugeordnet: Stufe 1: ,Digitale Minimalisten': 0 bis 30 Punkte; Stufe 2: ,Digital Konservative': 31 bis 50 Punkte; Stufe 3: ,Digitale Pragmatiker': 51 bis 70 Punkte; Stufe 4: ,Digital Fortgeschrittene': 71 bis 90 Punkte; Stufe 5: ,Digitale Vorreiter': 91 bis 100 Punkte. Diese Setzung berücksichtigt, dass in jeder Bildungsorganisation bereits ein ,Grundstock' an digitaler Reife vorherrschen sollte, sodass die niedrigste Kategorie breiter ausfallen muss. Umgekehrt sollen aber nur solche Mitarbeitende/Bereiche in die höchste Kategorie eingeordnet werden, die mind. $90 \%$ des erreichbaren Reifegrades aufweisen.

Die Online-Erhebung fand vom 25. Juni bis 20. Juli 2018 statt. In diesem Zeitraum nahmen insgesamt $N=171$ Personen teil. Etwas mehr als die Hälfte der Personen nimmt eine Lehrtätigkeit wahr, 32,7 \% haben eine Leitungsfunktion inne. Mehr als die Hälfte der Teilnehmenden besitzt einen Hochschulabschluss. Die Spanne der Berufserfahrung reicht von 1 bis 46 Jahren, wobei das arithmetische Mittel 19,57 Jahre beträgt. 35,1 \% der Teilnehmenden sind männlich, 59,6 \% weiblich, 9 Personen machten dazu keine Angabe. 


\subsection{Ergebnisse}

FF 1: Nutzung digitaler Medien

Bei der Nutzung digitaler Medien wird zwischen beruflicher und privater Nutzung unterschieden. Tabelle 3 zeigt die Ergebnisse im Überblick.

Tab. 3: Berufliche und private Nutzung digitaler Medien

\begin{tabular}{|c|c|c|c|c|c|c|c|}
\hline & \multicolumn{2}{|c|}{ beruflich } & \multicolumn{2}{|c|}{ privat } & \multirow[b]{2}{*}{$Z$} & \multirow[b]{2}{*}{$p$} & \multirow[b]{2}{*}{$r$} \\
\hline & $n$ & $M(S D)$ & $n$ & $M(S D)$ & & & \\
\hline $\begin{array}{l}\text { Computer, } \\
\text { Notebook, Laptop }\end{array}$ & 171 & $\begin{array}{c}4.92 \\
(.497)\end{array}$ & 168 & $\begin{array}{c}4.21 \\
(1.004)\end{array}$ & -7.040 & $0.000^{* * *}$ & .54 \\
\hline E-Mail & 170 & $\begin{array}{l}4.90 \\
(.506)\end{array}$ & 168 & $\begin{array}{l}4.63 \\
(.653)\end{array}$ & -4.464 & $0.000^{* * *}$ & .34 \\
\hline Standard-Software & 169 & $\begin{array}{c}4.82 \\
(.642)\end{array}$ & 166 & $\begin{array}{c}3.55 \\
(1.163)\end{array}$ & -9.088 & $0.000^{* * *}$ & .71 \\
\hline Spezialsoftware & 168 & $\begin{array}{c}4.20 \\
(1.244)\end{array}$ & 163 & $\begin{array}{c}1.72 \\
(1.208)\end{array}$ & -9.642 & $0.000^{* * *}$ & .76 \\
\hline Smartphone & 166 & $\begin{array}{c}3.36 \\
(1.766)\end{array}$ & 164 & $\begin{array}{l}4.90 \\
(.556)\end{array}$ & -7.926 & $0.000^{* * *}$ & .62 \\
\hline Messenger-Dienste & 168 & $\begin{array}{c}2.80 \\
(1.717)\end{array}$ & 168 & $\begin{array}{l}4.70 \\
(.919)\end{array}$ & -9.239 & $0.000^{* * *}$ & .72 \\
\hline Cloud-Dienste & 165 & $\begin{array}{c}2.17 \\
(1.509)\end{array}$ & 166 & $\begin{array}{c}2.64 \\
(1.553)\end{array}$ & -3.747 & $0.000^{* * *}$ & .29 \\
\hline $\begin{array}{l}\text { Streaming-Dienste, } \\
\text { Unterhaltungs-SW }\end{array}$ & 171 & $\begin{array}{c}1.85 \\
(1.106)\end{array}$ & 169 & $\begin{array}{c}3.57 \\
(1.257)\end{array}$ & -9.695 & $0.000^{* * *}$ & .75 \\
\hline Tablet/iPad & 169 & $\begin{array}{c}1.50 \\
(1.211)\end{array}$ & 167 & $\begin{array}{c}2.98 \\
(1.754)\end{array}$ & -8.200 & $0.000^{* * *}$ & .64 \\
\hline $\begin{array}{l}\text { Spiele-Software, } \\
\text { Spiele-Apps }\end{array}$ & 171 & $\begin{array}{l}1.09 \\
(.483)\end{array}$ & 169 & $\begin{array}{c}2.41 \\
(1.545)\end{array}$ & -8.140 & $0.000^{* * *}$ & .63 \\
\hline
\end{tabular}

Anm.: 1 = nie; 2 = seltener als $1 x$ monatlich; $3=$ mind. $1 x$ monatlich; $4=$ mind. $1 x$ wöchentlich; 5 = täglich; Wilcoxon-Test für abhängige Stichproben.

Quelle: eigene Darstellung.

Es zeigen sich über alle Technologien und Werkzeuge hinweg hochsignifikante Unterschiede zwischen beruflicher und privater Nutzung von mittlerer bis großer Effektstärke. Auffällig ist zudem, dass im beruflichen Kontext die Nutzung ,traditioneller' Medien weit überwiegt.

Hinsichtlich der Beurteilung der Mediennutzung wurde ebenfalls zwischen beruflicher und privater Nutzung differenziert. Tabelle 4 zeigt die Ergebnisse. 
Tab. 4: Bewertung der Nutzung digitaler Medien

\begin{tabular}{|c|c|c|c|c|c|c|c|}
\hline & \multicolumn{2}{|c|}{ beruflich } & \multicolumn{2}{|c|}{ privat } & \multirow[b]{2}{*}{$Z$} & \multirow[b]{2}{*}{$p$} & \multirow[b]{2}{*}{$r$} \\
\hline & $n$ & $M(S D)$ & $n$ & $M(S D)$ & & & \\
\hline $\begin{array}{l}\text { Die Nutzung von IT } \\
\text { und digitalen } \\
\text { Medien macht mir } \\
\text { Spaß. }\end{array}$ & 171 & $\begin{array}{c}4.72 \\
(1.134)\end{array}$ & 171 & $\begin{array}{c}4.89 \\
(1.052)\end{array}$ & -2.303 & $0.021^{*}$ & .18 \\
\hline $\begin{array}{l}\text { Ich möchte IT und } \\
\text { digitale Medien } \\
\text { intensiver nutzen. }\end{array}$ & 171 & $\begin{array}{c}4.28 \\
(1.390)\end{array}$ & 171 & $\begin{array}{c}3.94 \\
(1.423)\end{array}$ & -4.501 & $0.000^{* * *}$ & .34 \\
\hline
\end{tabular}

Anm.: $\quad$ Skala von $1=$,trifft überhaupt nicht zu“ bis $6=$,trifft vollkommen zu“; Wilcoxon-Test für abhängige Stichproben.

Quelle: eigene Darstellung.

Die Nutzung digitaler Medien ist demnach sowohl im beruflichen als auch privaten Bereich tendenziell mit positiven Emotionen verbunden, wobei der Unterschied zugunsten der Privatnutzung nur von geringer Effektstärke ist. Der Wunsch, IT und digitale Medien intensiver zu nutzen, ist dagegen im beruflichen Kontext spürbar deutlicher ausgeprägt (mittlerer Effekt).

FF 2: Einstellung zur Digitalisierung

Unterschiedliche Aspekte der Einstellung zur Digitalisierung zeigt Tabelle 5.

Tab. 5: Einstellung zur Digitalisierung

\begin{tabular}{lcc} 
& $n$ & $M(S D)$ \\
\hline $\begin{array}{l}\text { Die Digitalisierung eröffnet große Chancen zur } \\
\text { Weiterentwicklung von Wirtschaft und Gesellschaft. }\end{array}$ & 162 & $4.98(.909)$ \\
$\begin{array}{l}\text { Die Digitalisierung bietet mir große Potenziale zur beruflichen } \\
\text { Weiterentwicklung. }\end{array}$ & 162 & $4.58(1.050)$ \\
$\begin{array}{l}\text { Die Digitalisierung bringt große Gefahren mit sich. } \\
\begin{array}{l}\text { Die Digitalisierung bietet mir große Potenziale zur } \\
\text { persönlichen Weiterentwicklung. }\end{array}\end{array}$ & 162 & $4.48(1.138)$ \\
$\begin{array}{l}\text { Die Digitalisierung führt zu zusätzlichen Belastungen in } \\
\text { meinem Beruf. }\end{array}$ & 164 & $4.33(1.229)$ \\
$\begin{array}{l}\text { Die Digitalisierung bedeutet zusätzlichen Stress in meiner } \\
\text { alltäglichen Lebensgestaltung. }\end{array}$ & 163 & $3.46(1.564)$ \\
\hline
\end{tabular}

Quelle: eigene Darstellung.

Die Einstellungen zur Digitalisierung zeigen ein differenziertes Bild. Die Teilnehmenden sehen einerseits Chancen zur Weiterentwicklung auf individueller und gesellschaftlicher Ebene, andererseits aber auch Gefahren. 
FF 3: Digitaler Reifegrad

Die Berechnung der Teilindizes in den unterschiedlichen Modelldimensionen führt zu folgenden Ergebnissen (Tabelle 6):

Tab. 6: Mittlere Punktzahlen in den Dimensionen sowie mittlerer individueller Gesamtscore

\begin{tabular}{lcc} 
Dimension & $n$ & $M(S D)$ \\
\hline Mitarbeitende & 167 & $64.44(11.85)$ \\
Ausstattung und Technik & 171 & $62.66(16.53)$ \\
Strategie und Führung & 153 & $53.99(25.43)$ \\
Organisation & 163 & $47.36(28.80)$ \\
Kultur & 165 & $74.48(14.86)$ \\
Digitales Lehren und Lernen & 156 & $53.72(30.55)$ \\
Individueller Gesamtscore & 137 & $61.38(12.78)$ \\
\hline
\end{tabular}

Anm.: Abweichungen in der Stichprobengröße $n$ ergeben sich durch das Bewertungsverfahren, das eine Mindestzahl an beantworteten Items pro Dimension vorsieht.

Quelle: eigene Darstellung.

Insgesamt ergibt sich für die Mitarbeitenden-Dimension ein Reifegrad von 64.44 Punkten, was der Stufe 3 entspricht. Diese Reifestufe wird in den meisten anderen Dimensionen ebenfalls erreicht. Hervorzuheben ist dabei die Kultur-Dimension, die der Reifestufe 4 zugeordnet werden kann. Am schwächsten schneidet die Dimension Organisation ab. Mit 47.36 Punkten im Mittel befindet sich diese auf der Reifestufe 2. Insgesamt wird im Mittel ein individueller Gesamtscore von 61.38 Punkten erreicht, was der Reifestufe 3 entspricht.

Die Punktzahlen der Teilnehmer*innen variieren teilweise stark (Min 26.60, Max 94.28 Punkte). Im KBW wird hinsichtlich der individuellen Gesamtscores am häufigsten die Reifestufe 3 Digitale Pragmatiker erreicht. Die Verteilung über die definierten Reifestufen zeigt Abbildung 2. 
Abb. 2: Verteilung der Teilnehmenden über die definierten Reifestufen

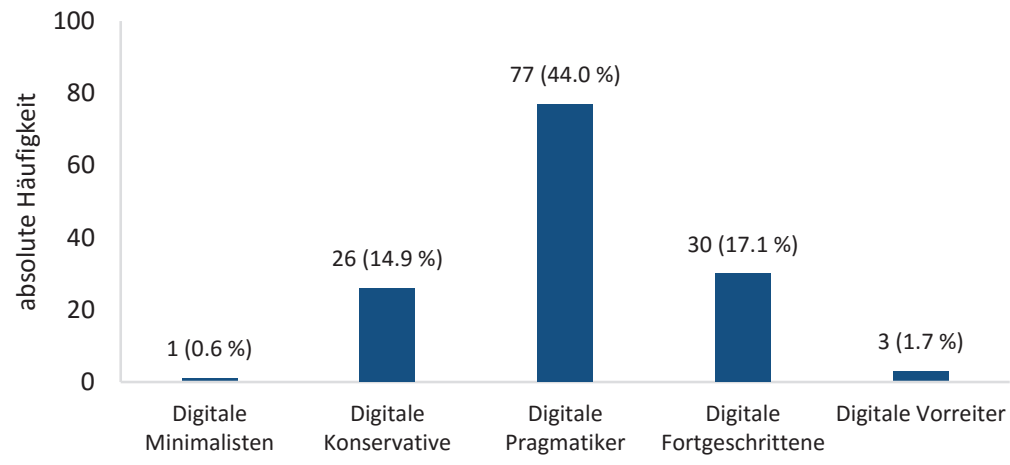

Quelle: eigene Darstellung.

Um die Auswirkungen von individuellen Faktoren auf die ,digitale Reife' der Mitarbeitenden zu analysieren, werden im Folgenden nur die Punktzahlen in der Mitarbeitenden-Dimension betrachtet. Signifikante Unterschiede zwischen Mitarbeitenden mit Lehrtätigkeit und Mitarbeitenden ohne Lehrtätigkeit gibt es nicht $(t(165)=.893, p=.373)$. Allerdings weisen Mitarbeitende, die eine Leitungsfunktion innehaben, eine signifikant höhere ,digitale Reife ' auf $(t(165)=2.948, p=.004)$. Weiterhin zeigt sich ein signifikanter Mittelwertsunterschied zwischen den Geschlechtern $(t(99.17)=2.170, p=.032)$. Der Mittelwert fällt bei männlichen Teilnehmenden höher aus $(M=67.60, S D=13.38)$ als bei weiblichen $(M=63.20, S D=10.38)$. Für den Bildungsabschluss und die Berufserfahrung ergeben sich hingegen keine signifikanten Unterschiede $(F(5,158)=.588, p=.709$ bzw. $F(3,163)=.300, p=.825)$.

\section{Diskussion und Ausblick}

In diesem Beitrag wurden die Entwicklung und Anwendung eines Reifegradmodells der Digitalisierung für Bildungsorganisationen nachgezeichnet. Die Operationalisierung erfolgte dabei über die Befragung der Mitarbeitenden eines Bildungswerks in Nordrhein-Westfalen.

In der betrachteten Stichprobe erscheinen die Mitarbeitenden als digitale Pragmatiker. Der Gesamtreifegrad der Organisation liegt ebenfalls auf dieser Stufe. Bei den befragten Mitarbeitenden bestehen geschlechtsspezifische Differenzen (im Mittel höhere Werte bei Männern) sowie Unterschiede in Bezug 
auf eine ausgeübte Leitungsfunktion (im Mittel höhere Werte bei Führungskräften). Systematische Unterschiede auf Basis von Bildungsabschluss und Berufserfahrung sowie ausgeübter Lehrtätigkeit bestehen hingegen nicht. Etwaige Unterschiede zwischen verschiedenen Unternehmensteilen können mit der ersten Stichprobe nur vermutet, aber nicht hinreichend plausibel analysiert werden.

Im ,Digitalisierungsbarometer ${ }^{6}$ sind hochsignifikante Unterschiede zwischen privater und beruflicher Nutzung digitaler Technologien von mittlerer bis großer Effektstärke zu verzeichnen. Mobile Geräte, Messenger- und VideoDienste werden kaum beruflich genutzt, so dass hier Potenziale zu vermuten sind. Die Nutzung von IT und digitalen Medien wird global durchaus positiv bewertet. Der Wunsch nach einem „Mehr“ ist dabei für den beruflichen Kontext deutlich ausgeprägter. Die Digitalisierung wird im Allgemeinen positiv bewertet, wobei auch deren Risiken nicht außer Acht gelassen werden. Stress und Belastung durch digitale Medien und IT erscheinen dabei im Mittel allerdings weniger virulent. In Summe kann von einem positiven Klima für Digitalisierungsprojekte im betrachteten Feld ausgegangen werden.

Die Ergebnisse des ,Digitalisierungsbarometers' bieten inhaltliche Anhaltspunkte für medienbezogene PE- und OE-Maßnahmen (so z. B. zu Themen wie videobasierter Lehre) sowie Anknüpfungspunkte für Medienentwicklungsprojekte, die direkt aus den Bedarfen der Organisation heraus entstehen. Im Sinne guter OE-Praxis sollten alle medienbezogenen Interventionen begleitet und im Dialog mit den Beteiligten (weiter-)entwickelt werden. Im Projekt \#ko.vernetzt stehen hierzu Kapazitäten innerhalb des sogenannten digital learning lab zur Verfügung. Eine nachhaltige Verankerung bedingt allerdings einen innerorganisationalen Transfer, der, zumindest aus gegenwärtiger Sicht, eine Herausforderung darstellen dürfte.

Die theoriegeleitete Modellierung von sechs Dimensionen und fünf Reifestufen erscheint hinsichtlich der Modellierungsziele als geeignet und weist in dem gewählten Auflösungsgrad auch über den betrachteten Forschungskontext hinaus. In der vorgestellten Form ermöglicht das Modell eine Beschreibung des Ist-Zustands (bzw. einen Prä-Post-Vergleich), Gruppenvergleiche innerhalb der betrachteten Organisation sowie externe Vergleiche mit anderen Bildungsorganisationen. Aus den Ergebnissen können (medien-)pädagogische Handlungsempfehlungen abgeleitet werden. Die diskutierte Modellierung weist aber naturgemäß auch eine Reihe von Limitationen auf. So beruht das Reifegradmodell vorrangig auf Einschätzungen von Mitarbeitenden. Subjektive Sichtweisen sollten hier durch möglichst objektive Maße, die Individualperspektive durch die Organisationsperspektive ergänzt werden. Nächste Schritte wären demnach - neben einer Stichprobenerweiterung um die Ergebnisse der korrespondierenden Paper\&Pencil-Befragung zur Minimierung des Methodenbias - eine Erweiterung des Ansatzes um zusätzliche, die Organisa- 
tions- bzw. ,Digitalkultur' betreffende Elemente einer Führungskräftebefragung. Eine Ausdifferenzierung des Konstrukts der ,digitalen Reife', die auch zukunftsbezogene Aspekte einer sogenannten ,Digital Readiness' mit aufnimmt (vgl. Remane et al. 2017), erscheint hier sinnvoll. Sollte im Zuge der iterativen Weiterentwicklung des Modells ein präskriptiver Einsatz in den Mittelpunkt rücken, so müssten nach Röglinger, Pöppelbuß und Becker (2012) weitere Design-Prinzipien für Reifegradmodelle wie bspw. Entscheidungsregeln für die Ableitung von Verbesserungsmaßnahmen umgesetzt werden.

\section{Literatur}

Becker, J., Knackstedt, R. \& Pöppelbuß, J. (2009). Entwicklung von Reifegradmodellen für das IT-Management. Wirtschaftsinformatik, 51(3), 249-260.

Berghaus, S., Back, A. \& Kaltenrieder, B. (2016). Digital Maturity \& Transformation Report 2016. Online: http://crosswalk.ch/media/25663/digital-maturity-transformation-report-2016-mit-best-practices.pdf (11.04.2018).

Bundesministerium für Wirtschaft und Energie, BMWI. (2017). Monitoring-Report Wirtschaft DIGITAL 2017. Online: https://www.bmwi.de/Redaktion/DE/Publikationen/Digitale-Welt/monitoring-report-wirtschaft-digital-2017.pdf?_blob=publi cationFile\&v $=18$ (09.04.2018).

Carvalho, J. V., Pereira, R. H. \& Rocha, Á. (2019). A comparative study on maturity models for information systems in higher education institutions. In T. Antipova \& Á. Rocha (eds.), Digital Science. DSIC18 2018. Advances in Intelligent Systems and Computing, Vol. 850, (pp. 150-158). Cham: Springer.

Chanias, S. \& Hess, T. (2016). How digital are we? Maturity models for the assessment of a company's status in the digital transformation. Management Report, 2, 1-14. Online: https://www.wim.bwl.uni-muenchen.de/download/epub/mreport_2016_2. $\operatorname{pdf}(14.06 .2019)$.

Christiansen, S.-K. \& Gausemeier, J. (2010). Klassifikation von Reifegradmodellen. ZWF Zeitschrift für wirtschaftlichen Fabrikbetrieb, 105(4), 344-349.

De Bruin, T., Freeze, R., Kaulkarni, U. \& Rosemann, M. (2005). Understanding the Main Phases of Developing a Maturity Assessment Model. Proceedings of the 16th Australasian conference on information systems (ACIS), 109. Online: http://aisel.aisnet.org/cgi/viewcontent.cgi?article=1220\& context=acis2005 (14.06.2019).

Egeli, M. (Hrsg.). (2016). Erfolgsfaktoren von Mobile Business. Ein Reifegradmodell zur digitalen Transformation von Unternehmen durch Mobile IT. Wiesbaden: Springer.

Euler, D. \& Wilbers, K. (2018). Berufsbildung in digitalen Lernumgebungen. In R. Arnold, A. Lipsmeier \& M. Rohs (Hrsg.), Handbuch Berufsbildung, (S. 1-13). Wiesbaden: Springer.

Gerholz, K.-H. \& Dormann, M. (2017). Ausbildung 4.0: Didaktische Gestaltung der betrieblich-beruflichen Ausbildung in Zeiten der digitalen Transformation.bwp@, Berufs- und Wirtschaftspädagogik - online, 32, 1-22. Online: http://www.bwpat. de/ausgabe32/gerholz_dormann_bwpat32.pdf (13.05.2019). 
Härtel, M., Brüggemann, M., Sander, M., Breiter, A., Howe, F. \& Kupfer, F. (2018). Digitale Medien in der betrieblichen Berufsbildung. Medienaneignung und Mediennutzung in der Alltagspraxis von betrieblichem Ausbildungspersonal (BIBB Wissenschaftliche Diskussionspapiere Nr. 196). Online: https://www.bibb.de/ veroeffentlichungen/de/publication/download/9412 (13.05.2019).

Helbig, C., Egloffstein, M., Hofhues, S. \& Ifenthaler, D. (im Druck). Förderung beruflicher Medienkompetenzen in einer vernetzten Bildungsorganisation. Konzeption und Perspektiven aus der wissenschaftlichen Begleitung des Verbundprojekts \#ko.vernetzt. Tagungsband zum AG BFN-Fachforum Berufsbildung 4.0 (November 2017, Darmstadt).

Initiative D21. (2018). D21-Digital-Index 2017/2018. Jährliches Lagebild zur Digitalen Gesellschaft. Online: https://initiatived21.de/app/uploads/2018/01/d21-digitalindex_2017_2018.pdf (19.07.2018).

Lahrmann, G. \& Marx, F. (2010). Systematization of Maturity Model Extensions. In R. Winter, J. L. Zhao \& S. Aier (eds.), Global perspectives on design science research. 5th International Conference, DESRIST 2010, St. Gallen, Switzerland, June 4-5, 2010. Proceedings, (S. 522-525). Berlin, Heidelberg: Springer.

Marx, F., Wortmann, F. \& Mayer, J. H. (2012). Ein Reifegradmodell für Unternehmenssteuerungssysteme. Wirtschaftsinformatik, 54(4), 189-204.

Mittelstand 4.0. (2016). Mittelstand im Wandel. Wie ein Unternehmen seinen digitalen Reifegrad ermitteln kann. Online: https://kommunikation-mittelstand.digital/content/uploads/2017/01/Leitfaden_Ermittlung-digitaler-Reifegrad.pdf-Reifegrad. pdf (19.07.2018).

PricewaterhouseCoopers, PwC. (2014). Digitalisierungsbarometer. Online: https:/www.pwc.de/de/digitale-transformation/assets/pwc_digitalisierungsbarometer_2014.pdf (19.07.2018).

Remane, G., Hanelt, A., Wiesboeck, F. \& Kolbe, L. (2017). Digital maturity in traditional industries: an exploratory analysis. Paper presented at the 25th European Conference on Information Systems (ECIS), Guimarães, Portugal. Online: https://aisel.aisnet.org/ecis2017_rp/10/ (13.05.2019).

Röglinger, M., Pöppelbuß, J. \& Becker, J. (2012). Maturity models in business process management. Business Process Management Journal, 18(2), 328-346.

Schäfer, D., Wichmann, K. S., Vogel, R. \& Rossmann, A. (2015). Digital Transformation Report 2015. Online: http://www.neuland.digital/neuland/wp-content/uploads/2016/01/DTA_Report_2015.pdf (21.04.2018).

Schuh, G., Anderl, R., Gausemeier, J., ten Hompel, M. \& Wahlster, W. (2017). Industrie 4.0 Maturity Index. Die digitale Transformation von Unternehmen gestalten (acatech Studie). München: Herbert Utz.

Seufert, S., Guggemos, J., Tarantini, E. \& Schumann, S. (2019). Professionelle Kompetenzen von Lehrpersonen im Kontext des digitalen Wandels. Entwicklung eines Rahmenkonzepts und Validierung in der kaufmännischen Domäne. Zeitschrift für Berufs- und Wirtschaftspädagogik, 115(2), 312-339.

Wendler, R. (2012). The maturity of maturity model research: A systematic mapping study. Information and Software Technology, 54(12), 1317-1339.

Wilbers, K. (2016). Berufsbildung 4.0: Berufsbildung im Zeitalter der großen Digitalisierung. Berufsbildung, 159, 7-10. 


\section{Professionalisierung des schulischen Bildungspersonals in den Gesundheits- und Pflegeberufen - Zwischenfazit eines berufs- und wirtschaftspädagogischen Sonderweges}

Karin Reiber, Ulrike Weyland und Eveline Wittmann

\section{Professionalisierung in den Gesundheits- und Pflegeberufen - eine prozedurale Perspektive}

Parallel zur wachsenden gesellschaftlichen Bedeutung aller Gesundheits- und ganz besonders der Pflegeberufe gewinnen Fragen der Professionalisierung ihres Bildungspersonals zunehmend an Relevanz. Angesichts demographischer Entwicklung und medizin-technischen Fortschritts werden Pflege- und Gesundheitsberufe zu einem hoch anspruchsvollen beruflichen Tätigkeitsfeld. Analog dazu steigen Anspruch und Erwartungen an die Berufsbildung dieser Domänen seitens der Berufspraxis, der Professionsvertreter*innen und der Gesellschaft (vgl. Reiber, Weyland \& Zoyke 2017). Auch die Erfordernisse der pädagogischen Praxis im Berufsfeld verändern sich - von der Heterogenität der Auszubildenden über die Ausdifferenzierung von Ausbildungszielen bis hin zur verstärkten Ausrichtung auf Fort- und Weiterbildung (Weyland \& Kaufhold 2017). Der Begriff der Professionalisierung steht hier also für die „Entwicklung eines (...) Berufsfeldes“ einerseits und andererseits für die Ausbildung beruflicher Kompetenzen mit dem Ziel, einer „,bezahlten Tätigkeit, die besonderen Leistungsstandards unterliegt“" (Mieg 2016, 27), nachgehen zu können.

Mit der Diskussion um eine Professionalisierung der Pflege- und Gesundheitsberufe und ihres Bildungspersonals verbindet sich eine prozedurale Sichtweise auf eine Entwicklung, in der gesellschaftliche Erfordernisse und Rahmenbedingungen interagieren, mit den Anforderungen an Kompetenzen, Ressourcen und Strategien der beruflichen Domänen (vgl. Kälble 2017). Diese Perspektive auf Professionalität erlaubt es - im Gegensatz zu einer apodiktischen Setzung von Profession als normativem Maßstab - dem evolutionären Charakter dieses Prozesses gerecht zu werden, der immer auch eine Abgrenzung und Emanzipation von der Medizin als klassische Profession einerseits und eine Überwindung des historischen Paradigmas des Helfens im Sinne von Dienen andererseits darstellt (vgl. Bollinger 2012). 
Sowohl im Prozess der Verberuflichung und Professionalisierung der Gesundheits- und Pflegeberufe als auch in dem ihn begleitenden (wissenschaftlichen) Diskurs nehmen die Pflegeberufe eine Art Vorreiterfunktion ein, da sie die größte Berufsgruppe innerhalb der Gesundheitsberufe darstellen (vgl. Kälble 2017) und die Abgrenzungs- und Profilierungserfordernisse des eigenen Tätigkeitsfeldes sowie seiner Ausdifferenzierung als besonders vordringlich wahrgenommen wurden (vgl. Kälble 2012). Insofern als in diesem Kontext die Akademisierung häufig wenn nicht gar als Synonym, so zumindest als Indikator für eine Professionalisierung gesetzt wird (vgl. Gerlach 2012), nimmt die Pflege auch hier eine paradigmatische Rolle ein. Die Entwicklung und Verbreitung der Pflegewissenschaft sowie ihre Repräsentanz in unterschiedlichsten Studienangeboten ist deutlich stärker ausgeprägt als in den anderen Gesundheitsberufen (vgl. Ewers et al. 2012), und innerhalb der Pflegewissenschaft hat die Professionalisierungsdebatte einen vergleichsweise hohen Stellenwert (vgl. Seltrecht 2016).

Parallel zu dieser Diskussion wurde und wird die Frage nach der damit korrespondierenden Professionalität des schulischen Lehrpersonals als eine notwendige, wenn auch nicht hinreichende Bedingung für die Professionalisierung der Pflegeberufe verhandelt. Komplementär zu den Professionalisierungsbestrebungen des gesamten Berufsstandes stehen auch hier die spezifischen Aufgaben und Kompetenzen in ihrem Spannungsgefüge zur Diskussion. Der hier vorliegende Beitrag bildet ein Symposium der Jahrestagung der Sektion Berufs- und Wirtschaftspädagogik 2018 ab und stellt dessen theoretisch-systematische Beiträge in den Kontext einer wissenschaftlichen Diskussion zum Thema „Professionalisierung des schulischen Bildungspersonals der beruflichen Fachrichtung Pflege“. Anknüpfend an die Fragestellung nach den Hintergründen einer verspäteten Professionalisierung von beruflicher Sorge- und Pflegearbeit im Allgemeinen sowie ihres schulischen Bildungspersonals im Besonderen hat der Beitrag zum Ziel, Merkmale dieses Sonderweges zu beleuchten und vor dem Hintergrund kollektiver und subjektiver Selbstvergewisserung zu diskutieren. Er behandelt am Beispiel der beruflichen Fachrichtung Pflege und mit Blick auf die strukturelle Rahmung der Lehrer*innenbildung die Frage, wie sich im Spannungsgefüge von verspäteter Professionalisierung einerseits und ökonomischen und sozialen sowie technischen Entwicklungen andererseits die professionelle Wissensbasis des schulischen Bildungspersonals entwickelt.

Zunächst wird dazu darauf eingegangen, wie im Zusammenhang mit Veränderungen im Berufsfeld auch die erforderliche Professionalisierung des Bildungspersonals diskutiert wurde und diese strukturelle und institutionelle Veränderungen induzierte. Sodann wird die Wissensbasis im Kontext der spezifischen Merkmale von Sorgearbeit und von Genderstrukturen im Aus- 
bildungs- und Beschäftigungssystem problematisiert, insbesondere im Kontext der ökonomischen Positionierung der Berufsgruppe und technologischer Entwicklungen. In Ermangelung entsprechender empirischer Forschungsergebnisse zur individuellen Entwicklung professioneller Wissensbezüge bleiben beide Diskussionen auf die strukturelle Ebene beschränkt. Abschließend wird gefragt, inwieweit durch den Fachqualifikationsrahmen Pflegedidaktik (Walter \& Dütthorn 2019), der als Element professioneller und disziplinärer Selbstvergewisserung bezüglich der Professionalisierung des schulischen Bildungspersonals für die Domäne Pflege verstanden wird, ein Beitrag zur Bearbeitung der aufgeworfenen Problematiken geleistet werden kann.

\section{Hintergründe und Legitimation der Professionalisierung von schulischem Bildungspersonal für die pflegeberufliche Bildung}

Die Beschäftigung mit Professionalisierung ist zugleich auch immer die Frage nach der Wissensbasis von Berufen und ihrer Realisierung in Form der Berufsausbildung (vgl. Seltrecht 2016). In professionssoziologischer Perspektive sind Ausbildungsstrukturen und -inhalte einerseits Ausdruck von Professionalisierung als kollektivem Prozess, in dem die Berufsgruppen ihre Strategien der Ausgestaltung von beruflicher Handlungsautonomie im Hinblick auf gesellschaftliche Bedarfe und Anforderungen ausüben. Sie bilden andererseits den Ausgangs- und Bezugsrahmen für Professionalisierung im Sinne einer individuellen Kompetenzentwicklung. Insofern lässt sich auch die Entwicklung und Ausdifferenzierung der pflegeberuflichen Ausbildung auf berufsfachschulischem Niveau als ein Prozess der Professionalisierung fassen, der zwar immer wieder durch Deprofessionalisierungsereignisse gestört wurde, insgesamt aber dennoch als ein Fortschritt hin zu mehr professioneller Kompetenz und Autonomie gelesen werden kann (vgl. ebd.).

In toto kann für alle Gesundheits- und Pflegeberufe konstatiert werden, dass sie im Hinblick auf ihre Verortung im Berufsbildungssystem und ihre Regelungen eine Sonderstellung einnehmen, insofern als sie außerhalb des Berufsbildungsgesetzes über Berufszulassungsgesetze geregelt werden (vgl. Reiber et al. 2017). Neben diesem starken Fokus innerhalb der Professionalisierungsdebatte der Gesundheits- und Pflegeberufe auf die Domäne der Pflege, und hier insbesondere auf Fragen der Akademisierung, gibt es eine zweite Diskussionslinie zur Lehrtätigkeit und zum Berufsbildungspersonal. Bereits seit den 1990er Jahren wurde insbesondere die Sonderstellung der Ausbildung des Lehrpersonals für die Gesundheitsberufe im Allgemeinen (Bals 
1992, 1993) und die Pflegeberufe im Besonderen (Bischoff 1993; Wanner 1993) thematisiert. Dieser Diskurs fokussierte auf die Abweichung von den sonst üblichen Standards - die Lehrkräftequalifizierung fand in Form beruflicher Weiterbildung anstelle eines wissenschaftlichen Studiums statt - und skizzierte die zukünftige Lehrer*innenbildung als originär berufspädagogische und pflegewissenschaftliche Hochschulbildung.

Die Diskussionslinie setzt sich begleitend zur Akademisierung der Lehrer*innenbildung für die berufliche Fachrichtung Pflege und der Umstellung von relativ neuen Studienangeboten auf die Bachelor- und Master-Struktur im Zuge des Bologna-Prozesses fort. Problematisiert wurden in diesem Zuge die ggf. damit einhergehenden Deprofessionalisierungstendenzen durch eine Fortsetzung von Sonderwegen (z. B. Bischoff-Wanner 2008; Reiber 2008; Darmann-Finck \& Ertl-Schmuck 2008). Zwischenbilanzierende Beiträge vertiefen dabei spezifische Aspekte der Professionalisierung wie z. B. Berufseinmündung, -verbleib und -entwicklung von Pflegepädagog*innen (Brühe 2013, 2016; Reiber, Winter \& Mosbacher 2012; Reiber, Winter \& Mosbacher-Strumpf 2015) oder spezifische berufsbiographische Besonderheiten und die daraus resultierenden hochschuldidaktischen Erfordernisse (z. B. Weyland \& Reiber 2013).

Ergänzend zu den o.g. forschungsbasierten Arbeiten sind hier deskriptiv akzentuierte Beiträge zum Thema „Lehrer*innenbildung der Pflege- und Gesundheitsberufe" zu nennen, die auf Strukturfragen und unterschiedliche Studienprofile lehrerbildender Studiengänge (so z. B. einige Beiträge in Arens 2018) fokussieren und den jeweiligen Status quo der Professionalisierung an unterschiedlichen Studienstandorten abbilden. Dies ist Ausdruck einer Situation, in der noch immer partikulare Sonderwege bei der Ausbildung des Berufsbildungspersonals zu konstatieren sind (vgl. Reiber 2018). Im Rahmen disziplinärer Verortungsbewegungen fand dieser Diskurs in den zurückliegenden zehn Jahren verstärkt auch innerhalb der Berufs- und Wirtschaftspädagogik statt, indem die Tagungen der Sektion kontinuierlich genutzt wurden, um im Rahmen von Symposien exemplarische Fragestellungen dieses Themenkreises zu präsentieren und diskutieren.

\section{Professionalisierung des schulischen Bildungspersonals der Pflegeberufe im Kontext von Care Work}

Aus der vorab vorgenommenen Exposition wird ein deutlicher Schwerpunkt der bisherigen Diskussion und Arbeiten zur Professionalisierung des schulischen Bildungspersonals der beruflichen Fachrichtungen Pflege und Gesund- 
heit erkennbar: Thematisiert wird die Lehrer*innenbildung mit Fokus auf Strukturen, wenngleich Konsequenzen für die Art der Wissensbasis erkennbar werden. Dies lässt sich unschwer damit erklären, dass Ausbildungsstrukturen ,als bedeutsamer Aspekt der Professionsentwicklung zu werten“ (Ortenburger 2016, 561) sind. In Erweiterung dieses Blickwinkels ist danach zu fragen, welche Wissensbereiche für die beruflichen Kernaufgaben erforderlich sind. Unterscheiden lässt sich dabei wiederum eine formal-kollektive und eine subjektbezogene Perspektive. Mit der erstgenannten Blickrichtung verbinden sich Aushandlungs- und Konsentierungsprozesse einer Berufsgruppe bezüglich konstitutiver Merkmale der Berufszugehörigkeit, mit der zweiten deren individuelle Aktualisierung in beruflichen Handlungsvollzügen (vgl. Meyer 2010).

Als paradigmatische Rahmung sowohl für die kollektive als auch für die individuelle Lesart von Professionalisierung ist es zielführend, sich zunächst den Charakter der Gesundheits- und Pflegeberufe im Besonderen in der Rahmung von Care Work im Allgemeinen zu vergegenwärtigen. Pflegeberufe repräsentieren in dieser Sichtweise die Besonderheiten aller personenbezogenen Dienstleistungsberufe insoweit, als sie sowohl ,kommunikative, interaktive und subjektbezogene Fähigkeiten fokussieren sowie ebenfalls (einer) sachbezogenen Logik folgen“" (Friese 2018, 32). Als typische Frauenberufe läuft Sorgearbeit aufgrund ihres historischen Erbes Gefahr, zu sehr auf allgemeine (meist weiblich konnotierte) Daseinskompetenzen reduziert zu werden (vgl. Friese 2017).

Verdeutlichen lässt sich dies laut Friese (2018) an der Vernachlässigung der Merkmale von Care Work in der aktuellen Diskussion um Industrie 4.0 und Digitalisierung - obwohl der gesamte Bereich personenbezogener Dienstleistungen wie Pflege und Kinderbetreuung in wirtschaftlicher Hinsicht hohe Wachstumsraten aufweist. Der „Beitrag von Care Work zur ökonomischen, sozialen und kulturellen Kapitalbildung" (a. a. O., 21) werde damit unterschätzt. Neben diesen Potenzialen ist jedoch vor dem historischen Hintergrund dieser Berufe als Frauenberufe vor weiteren Modernisierungsfallen zu warnen, und „,neu entstehende Beschäftigungsrisiken durch Deregulierung, Abwertung und Abdrängung von Care Work" (ebd.) sind kritisch zu beobachten.

Auch Ausbildungsstrukturen und Ordnungsrecht sind gekennzeichnet durch diese ,gendercodierten Berufsstrukturen“ (Friese 2018, 21) und kommen u. a. darin zum Ausdruck, dass es unterschiedliche und teilweise von den sonst üblichen Rahmenordnungen abweichende Zuständigkeiten gibt. Besonders deutlich wird dies an der Sonderstellung der Schulen für Pflegeund Gesundheitsberufe (vgl. auch Reiber et al. 2017) und die damit verbundenen Fragen zur Vergleichbarkeit mit Abschlüssen im Dualen System und 
zur Durchlässigkeit (vgl. Friese 2018). Zwar wird die durch das neue Pflegeberufegesetz initiierte Ausbildungsreform ab 2020 einerseits eine stärkere Vereinheitlichung der bisher nach unterschiedlichen Berufsgesetzen geregelten Pflegeberufe („Generalistik“) und andererseits ein diese berufsschulische Ausbildungsform ergänzendes, grundständiges primärqualifizierendes Studium in das Regelangebot überführen (vgl. Reiber et al. 2018), die Sonderstellung der Berufsfachschulen außerhalb der Zuständigkeit des BBiG ist damit jedoch noch nicht überwunden.

Nach Auffassung von Friese $(2018,32)$ ist auch in curricularer Hinsicht die bisherige Entwicklung geprägt von der „Ausblendung von Genderstrukturen und Sorgearbeit im Ausbildungs- und Beschäftigungssystem“. Demgegenüber hieße Professionalisierung, Curricula weiterzuentwickeln, um so Sorgearbeit ,im Spannungsfeld von ökonomischen und sozialen sowie technischen Bedingungsfaktoren“ (a. a. O., 33) neu zu kontextuieren. Die für Pflege- und Gesundheitsberufe konstitutive, fachlich durchdrungene soziale und personale Kompetenz (vgl. Wittmann et al. 2014; Kaspar et al. 2016; Wittmann, Kaspar \& Döring 2017) ist dabei curricular ebenso abzubilden wie das fachlich und methodisch stabil abgesicherte Handlungswissen.

Im Hinblick auf die expandierenden Studienangebote der Lehrer*innenbildung für die die berufliche Fachrichtung Pflege werden zwar „Modernisierungslücken aufgeholt“ (Friese 2018, 38). Auch sind erste hochschulische Angebote für das Berufsbildungspersonal in Entwicklung, die die Schnittstelle zwischen Pflege, technologischer Entwicklung und Berufs/Pflegepädagogik thematisieren (Weyland \& Kaufhold 2017, 32; Wittmann \& Flemming 2018). Jedoch besteht weiterhin ,Entwicklungsbedarf [...] bezüglich der bundeseinheitlichen Homogenisierung von Studienprofilen, Benennungen und Abschlüssen sowie Anrechnungsverfahren und Ausbildungsniveaus" (Friese 2018, 38). Diese von Friese vor dem Hintergrund der originären Lehramtsstudiengänge formulierte Forderung lässt sich im Hinblick auf die zahlreichen daneben existierenden Studienangebote mit den unterschiedlichsten Bezeichnungen (z. B. Pflegepädagogik, Berufspädagogik der Gesundheitsberufe), in den verschiedensten Organisationsformen (von Vollzeit über berufsbegleitend bis hin zum Fernstudium) und mit diversen Abschlüssen (Bachelor und Master of Arts oder Science) doppelt unterstreichen. ${ }^{1}$ Der im neuen Pflegeberufegesetz nun endlich verbindlich festgeschriebene Mastergrad als Regelabschluss für die Lehrtätigkeit ist hier zwar einerseits überfällig und sehr zu begrüßen. Die Regelung kann aber auch zu einem weiteren

1 Auf eine weitere Besonderheit und Abweichung von den sonst üblichen Studienstrukturen, nämlich dass diese Formen der Lehrer*innenbildung ohne institutionalisierte zweite und dritte Phase ablaufen, kann an dieser Stelle leider nicht weiter eingegangen werden. 
Wildwuchs an Studienangeboten als Reaktion auf den hohen (Nach-)Qualifizierungsbedarf von Personen führen, die aufgrund ihrer spezifischen berufsbiographischen Bedingungen ihre Lehrer*innenbildung neben anderen Verpflichtungen wie Berufstätigkeit und/oder familiäre Pflichten komplettieren möchten bzw. müssen. Damit würden aus einer gesellschaftlichen Anforderung - wie dem qualitativ und quantitativ gestiegenen Bedarf an pflegerischer Versorgung - resultierende überfällige Entwicklungen individualisiert und angesichts des hohen Frauenanteils im Pflegeberuf gendertypische Berufsstrukturen auch im Bereich des schulischen Bildungspersonals zementiert.

\section{Professionelle Selbstvergewisserung zwischen Standardisierung und Ambiguität}

Vor dem Hintergrund dieser divergierenden Situation bezogen auf die Lehrer*innenbildung und in Anerkennung der aus den Spezifika von Care Work resultierenden Ambiguität als Professionsmerkmal wird im nächsten Schritt exemplarisch für eine kollektive Bestimmung von professionellen Kompetenzen für das Berufsbildungspersonal der Domäne Pflege - der Fachqualifikationsrahmen Pflegedidaktik vorgestellt (Walter \& Dütthorn 2019). Dieser beruht auf einem mehrjährigen Aushandlungsprozess von Wissenschaftler*innen, die auf diesem Gebiet forschen und arbeiten. Ziel des Qualifikationsrahmens ist die Verständigung auf grundlegende Kompetenzen für die Handlungsfelder der Pflegebildungspraxis und deren wissenschaftliche Reflexion. Der daraus resultierende Referenzrahmen für die Pflegelehrer*innenbildung schreibt sich dabei selbst „eine strukturelle und wissenschaftlich fundierte Orientierungs- und inhaltliche Legitimationsfunktion" (a. a. O., 29) zu, jedoch ohne eine „rigide Normierung von Studienprogrammen und -inhalten“" (a. a. O., 6) zu beabsichtigen.

\subsection{Der Fachqualifikationsrahmen Pflegedidaktik}

Zur näheren Bestimmung eines spezifischen Kompetenzprofils wurde ein Referenzrahmen für die fachdidaktischen Anteile der Lehrer*innenbildung erarbeitet, der die analytischen Fertigkeiten ebenso integriert wie reflexivfallbezogene Fähigkeiten. Innerhalb der vorgegebenen Rahmung von übergeordneten Ordnungssystematiken wie EQR, DQR und HQR und auf Basis einer disziplinären Selbstvergewisserung im Beziehungsgefüge von Pflegewissenschaft und Pflege(bildungs)praxis einerseits, der Erziehungswissenschaft und ihren Subdisziplinen Berufs- und Wirtschaftspädagogik sowie Erwachsenenbildung anderseits, wurden Kompetenzen identifiziert, die den 
o. g. Besonderheiten von Sorgearbeit Rechnung tragen. Dabei wurden auch die Lehrerbildungsstandards der Kultusministerkonferenz (KMK) sowie das Basiscurriculum der Berufs- und Wirtschaftspädagogik „,diskutiert und für mehr oder weniger richtungsweisend befunden" (Walter \& Dütthorn 2019, 9). Als Ergebnis dieser Diskussion ist festzuhalten, dass die Standards für die Lehrer*innenbildung und das Basiscurriculum Berufs- und Wirtschaftspädagogik nicht explizit hinsichtlich der dort aufgeführten Kompetenzen aufgegriffen werden (vgl. ebd.), weil „der FQR Pflegedidaktik nicht alle Studienbereiche thematisiert, sondern ausschließlich die spezifisch pflegedidaktischen" (ebd.). Hingegen wurden die ländergemeinsamen Anforderungen der KMK für die Fachwissenschaften und -didaktiken der beruflichen Fachrichtung Pflege $(2018$, 86-88) zugrunde gelegt und konkretisiert, da sie die Besonderheiten des Berufs und die grundlegenden Kompetenzen für die Lehrenden dieses Bereichs abbilden.

In Anlehnung an eine strukturtheoretische Bestimmung von Professionalität pädagogischen Handelns in der Pflege wird diese als potenziell von Antinomien und Paradoxien mit doppeltem Handlungsbezug im Spektrum von sozialen, ökonomischen und technologischen Anforderungen bestimmt: Sie bezieht sich auf berufliche Situationen, die in sich widersprüchlich sein können (z. B. individuelles Bedürfnis vs. organisationale Erfordernis) und zu paradoxen Handlungsanforderungen führen können und agiert selbst zugleich in diesem Spannungsgefüge (vgl. Seltrecht 2015).

Die Grundstruktur des FQR Pflegedidaktik wird innerhalb der grundlegenden Leitprinzipien für Hochschulbildung entfaltet; hier werden die Wissenschafts- und die Bildungs- resp. Subjektorientierung sowie die Innovations- und Professionsorientierung genannt. Kurzgefasst bedeutet dies, dass Lehrer*innen-Bildung als Hochschulstudium zugleich wissenschaftlich fundiert und für das spätere berufliche Handeln relevant sein sollte sowie ein Beitrag zur Persönlichkeitsentwicklung und die Grundlegung für eine kontinuierliche Kompetenzentwicklung leisten sollte. Innerhalb dieser Bezüge sind die Kompetenzen für die Makro-, Meso- und Mikroebene des pflegedidaktischen Handelns operationalisiert, und jeder Ebene sind wiederum Reflexions- und Handlungsfelder zugeordnet. Die jeweiligen Kompetenzen sind sowohl für die Bachelor- als auch für die Masterstufe ausformuliert (vgl. Walter \& Dütthorn 2019).

Dies kann an einem Beispiel veranschaulicht werden. Auf der Mikroebene wird als eines von drei Handlungs- und Reflexionsfeldern die Durchführung von Lehr-Lernsituationen definiert. Es ,umfasst die konkrete Durchführung von Lehr-Lernsituationen an allen pflegeberuflichen Lernorten (Bildungsinstitution, Institutionen beruflicher Pflege und dritter Lernort). Im Zentrum steht die Gestaltung pädagogischer Interaktionen und Beziehungen zu den Teilnehmenden, Lernenden bzw. Studierenden." (Walter \& Dütthorn 2019, 26). Dies wird wiederum operationalisiert zu Kompetenzbeschreibun- 
gen für die beiden formalen Qualifikationsniveaus. Ein Beispiel hierfür sind folgende Formulierungen: Die Absolvent*innen ,...entwickeln ein hermeneutisches Fallverständnis - d. h. sie berücksichtigen pflegespezifische emotionale und leibbezogene Lerngegenstände und finden dadurch Anschlüsse an die Lebens- bzw. die pflegerische Berufswelt der Lernenden und deuten diese vor dem Hintergrund pflege- und bezugswissenschaftlicher Erkenntnisse" (a. a. O.) (Bachelorebene); sie „... sind für die zentrale Bedeutung der Antinomien des professionellen pflegedidaktischen und pflegerischen Handelns sensibilisiert, identifizieren und reflektieren typische individuelle und kollektive Reaktionsmuster und verfügen über einen reflexiven Umgang mit diesen Antinomien“"(a. a. O.) (Masterebene).

Die Bedeutung dieses Fachqualifikationsrahmens ist im Kontext der hier vorliegenden Fragestellung darin zu sehen, dass er einen Verständigungsprozess der Scientific Community bezüglich der Kernaufgaben und der dafür erforderlichen Wissensbestände, Fähigkeiten und Fertigkeiten abbildet. Ganz generell und unabhängig von diesem Beispiel ist zu konstatieren, dass eine curricular systematisch angelegte Fachdidaktik der beruflichen Fachrichtung Pflege einen wichtigen Beitrag im Hinblick auf eine weitere Professionalisierung sowohl der Lehrtätigkeit als auch der Profilbildung und Normalisierung der Lehrer*innen-Bildung (vgl. Reiber 2018) leisten kann.

\subsection{Umsetzungsbarrieren auf der individuellen Handlungsebene?}

Ausgehend von einer historisch-kritischen Bilanzierung der Professionalisierung personenbezogener Dienstleistungsberufe sowie der darauf bezogenen Lehrer*innenbildung wurde hier ein Diskussions- und Verständigungsprozess auf fachdidaktische Ausbildungsstandards als „kollektive Orientierung“ (Meyer 2010, 3) im Rahmen von Professionalisierung vorgestellt. Komplementär dazu stellt sich die Frage nach Professionalität als ,subjektbezogene Kategorie(n) auf der individuellen Handlungsebene" (a. a. O., 4; kursiv im Original) und somit nach der Relevanz dieser formal-kollektiven Seite von Professionalisierung für subjektive Deutungsherausforderungen in ihrer individuellen berufsbiografischen Rahmung.

Erste Hinweise dazu finden sich beispielsweise bei Walter (2018), die diesbezüglich 17 Studierende eines berufsbegleitenden Masterstudiengangs für Berufspädagogik der Gesundheitsberufe befragt. Im Rahmen einer Gruppendiskussion reflektieren die Studierenden ihre didaktische Kompetenzentwicklung im Spiegel fach- und berufsfelddidaktischer Studienanteile. Im Spannungsfeld des lebensweltlichen Bezugs der Praxis und des Wissenschaftsbezugs von Theorie geraten die Studierenden demzufolge mitunter in Deutungskonflikte. Sie haben einerseits den Anspruch, „soeben Gelerntes unmittelbar in ihren pädagogischen Berufsalltag integrieren“ (Walter 2008, 
96) zu können. Andererseits oszillieren die Studierenden zwischen Ehrfurcht und Kritik angesichts von Theorien und Modellen.

Die Ergebnisse einer auf Basis der Grounded Theory-Methodologie durchgeführten Studie von Simon (2018) deuten darüber hinaus an, dass sich auch bei erfahrenen Pflegelehrkräften ein Phänomen tendenzieller Vermeidung wissenschaftlicher Erkenntnisse in der Unterrichtplanung feststellen lässt. Die Befunde legen nahe, dass die geforderte Neukontextuierung von Pflegearbeit ,im Spannungsfeld von ökonomischen und sozialen sowie technischen Bedingungsfaktoren“ (Friese 2018, 33) durch Akademisierung des Bildungspersonals auch bei Einführung einheitlicher curricularer Standards an fach- und berufsfelddidaktische Grenzen keinen Selbstläufer darstellt.

\section{Fazit und Ausblick}

In diesem Beitrag wurde die Professionalisierung des schulischen Bildungspersonals in Pflegeberufen - paradigmatisch für die Gesundheitsberufe - als berufs- und wirtschaftspädagogischer Sonderweg unter Heraushebung der Pflegeberufe thematisiert. Dabei wurde besonders die Betonung genderspezifischer "Daseinskompetenzen“" in diesen Berufen herausgestellt und als Grund für Professionalisierungsdefizite auch des Bildungspersonals angesehen. Im Kontext ökonomischer und technologischer Rationalitäten kann dies als höchst problematisch angesehen werden. Formal-kollektive Konsentierungsprozesse der Wissensbasis für das schulische Bildungspersonal in den Gesundheits- und Pflegeberufen wären vor diesem Hintergrund wünschenswert und liegen in Form des Fachqualifikationsrahmens Pflegedidaktik ansatzweise vor, sind jedoch im Lichte des Selbstverständnisses der Studierenden wie auch der aktuellen Lehrkräfte in der Umsetzung in individuelle Handlungsstrukturen erwartbarerweise nicht trivial. Dieser Ausschnitt aus der Lehrer*innenbildung repräsentiert dementsprechend in erster Linie die mit einem hohen Nachholbedarf beim Auf- und Ausbau lehrerbildender Studiengänge (vgl. Friese 2018) verbundenen Sonderwege und -formen, die dann auch besondere hochschuldidaktische Professionalität seitens der Lehrenden an den Hochschulen erfordern (vgl. Weyland \& Reiber 2013). Auch wäre im Weiteren zu problematisieren, inwieweit der vorliegende Fachqualifikationsrahmen das aufgezeigte Spannungsfeld - auch in Anbetracht erwartbarer digitalisierungsbedingter Umwälzungen - bereits hinreichend thematisiert.

Für eine weitere Professionalisierung des schulischen Bildungspersonals in den Gesundheits- und Pflegeberufen bedürfen disziplin- und professionsspezifische Verständigungsprozesse über erforderliche curriculare Rahmensetzungen einer Weiterentwicklung, gerade auch mit Blick auf die aufgezeigten sorgearbeits- und genderspezifischen Spannungsfelder. Andererseits 
verweisen die Ausführungen auch mögliche Begrenzungen dieses Wegs bezogen auf den Transfer in individuelle Wissensentwicklungen. Inwieweit die Thematisierung und handlungsorientierte Bearbeitung dieser Spannungsfelder einen Beitrag auch zur individuellen Entwicklung der Wissensbasis leisten und damit zu Professionalisierungsprozessen auf der Subjektebene beitragen können, wäre ein zentraler Gegenstand für ein Forschungsprogramm zur Professionalisierung des betroffenen schulischen Bildungspersonals.

\section{Literatur}

Arens, F. (Hrsg.). (2018). Lehrerbildung der Gesundheitsberufe im Wandel. Von der Pflegepädagogik zur Berufspädagogik Pflege und Gesundheit. Berlin: wvb Wissenschaftlicher Verlag.

Bals, T. (Hrsg.). (1992). Professionalisierung des Lehrens im Berufsfeld Gesundheit. Köln: Müller Botermann.

Bals, T. (1993). Gesundheitslehrer oder Krankenpflegepädagogen? Zur Frage der Normalität der Lehrerausbildung in den Gesundheitsfachberufen. In C. Bischoff \& P. Botschafter (Hrsg.), Neue Wege in der Lehrerausbildung für Pflegeberufe, (S. 87-110). Melsungen: Bibliomed.

Bischoff, C. (1993). Pädagogische Überlegungen zu einer Rechtsform der Lehrerausbildung oder: Müssen wir alles neu erfinden? In C. Bischoff \& P. Botschafter (Hrsg.), Neue Wege in der Lehrerausbildung für Pflegeberufe, (S. 73-86). Melsungen: Bibliomed.

Bischoff-Wanner, C. (2008). Die Lehrerbildung in der Pflege im Zeichen von „Bologna“. In C. Bischoff-Wanner \& K. Reiber (Hrsg.), Lehrerbildung in der Pflege. Standortbestimmung, Perspektiven und Empfehlungen vor dem Hintergrund der Studienreformen, (S. 11-40). Weinheim, München: Beltz Juventa.

Bollinger, H. (2012). Profession - Dienst - Beruf. Der Wandel der Gesundheitsberufe aus berufssoziologischer Perspektive. In H. Bollinger, A. Gerlach \& M. Pfadenhauer (Hrsg.), Gesundheitsberufe im Wandel. Soziologische Betrachtungen und Interpretationen, (S. 13-30). Frankfurt am Main: Mabuse.

Brühe, R. (2013). Berufseinmündung von Pflegelehrern. Eine empirische Untersuchung zur Situation und zum Erleben von Pflegelehrenden an Pflegebildungseinrichtungen in der Phase der Berufseinmündung. Philosophisch-Theologische Hochschule Vallendar. Online: http://nbn-resolving.de/urn:nbn:de: 0295-opus1376 (13.12.2018).

Brühe, R. (2016). Berufseinmündung von Pflegelehrenden. In E. BrinkerMeyendriesch \& F. Arens (Hrsg.), Diskurs Berufspädagogik Pflege und Gesundheit, (S. 31-49). Berlin: wvb. 
Bundesgesetzblatt. (2017). Gesetz zur Reform der Pflegeberufe (Pflegeberufereformgesetz - PflBRefG). Vom 24.07.2017 (49). Online: https://www.bgbl.de/ xaver/bgbl/text.xav?SID $=\& \mathrm{tf}=$ xaver.component.Text_0\&tocf $=\& \mathrm{qmf}=\&$ hlf $=$ xave r.component.Hitlist_0\&bk=bgbl\&start=\%2F\%2F*\%5B $\% 40$ node_id $\% 3 \mathrm{D} \% 27263$ $238 \% 27 \% 5$ D\&skin $=$ pdf\&tlevel=-2\&nohist $=1$ (10.12.2018).

Darmann-Finck, I. \& Ertl-Schmuck, R. (2008). Strukturmodelle der Lehrerbildung im Bachelor-/Master-Studiensystem. In C. Bischoff-Wanner \& K. Reiber (Hrsg.), Lehrerbildung in der Pflege. Standortbestimmung, Perspektiven und Empfehlungen vor dem Hintergrund der Studienreformen, (S. 65-84). Weinheim, München: Beltz Juventa.

Walter, A. \& Dütthorn, N. (Hrsg.). (2019). Fachqualifikationsrahmen Pflegedidaktik. Sektion Bildung und Sektion Hochschullehre der Deutschen Gesellschaft für Pflegewissenschaft, Duisburg: Deutsche Gesellschaft für Pflegewissenschaft. Duisburg: Eigendruck.

Ewers, M., Grewe, T., Höppner, H., Huber, W., Sayn-Wittgenstein, F., Stemmer, R., Voigt-Radloff, S. \& Walkenhorst, U. (2012). Forschung in den Gesundheitsfachberufen. Potenziale für eine bedarfsgerechte Gesundheitsversorgung in Deutschland. Deutsche Medizinische Wochenschrift, 137, 37-73. Online: http://bdslev.de/files/2012_06_dmw_gfr_forschung_in_den_gesundheitsfachberufen_2_. $\operatorname{pdf}(28.10 .2018)$.

Friese, M. (2017): Care Work. Eckpunkte der Professionalisierung und Qualitätsentwicklung in personenbezogenen Dienstleistungsberufen. In U. Weyland \& $\mathrm{K}$. Reiber (Hrsg.), Entwicklungen und Perspektiven in den Gesundheitsberufen - aktuelle Handlungs- und Forschungsfelder, (S. 29-49). Bielefeld: Bertelsmann.

Friese, M. (2018). Berufliche und akademische Ausbildung für Care Berufe. Überblick und fachübergreifende Perspektiven der Professionalisierung. In M. Friese (Hrsg.), Reformprojekt Care Work. Professionalisierung der beruflichen und akademischen Ausbildung, (S. 17-44). Bielefeld: wbv.

Gerlach, A. (2012). Akademisierung ohne Professionalisierung? Die Berufswelt der ersten Pflegeakademikerinnen in Deutschland. In H. Bollinger, A. Gerlach \& M. Pfadenhauer (Hrsg.), Gesundheitsberufe im Wandel. Soziologische Betrachtungen und Interpretationen, (S. 71-102). Frankfurt am Main: Mabuse.

Kälble, K. (2012). Modernisierung durch wissenschaftsorientierte Ausbildung an Hochschulen. Zum Akademisierungs- und Professionalisierungsprozess der Gesundheitsberufe in Pflege und Therapie. In H. Bollinger, A. Gerlach \& M. Pfadenhauer (Hrsg.), Gesundheitsberufe im Wandel. Soziologische Betrachtungen und Interpretationen, (S. 31-53). Frankfurt am Main: Mabuse.

Kälble, K. (2017). Zur Professionalisierung der Pflege in Deutschland. Stand und Perspektiven. In T. Sander \& S. Dangendorf (Hrsg.), Akademisierung der Pflege. Berufliche Identitäten und Professionalisierungspotentiale im Vergleich der Gesundheits- und Sozialberufe, (S. 27-58). Basel, Weinheim: Beltz Juventa.

Kaspar, R., Döring, R., Wittmann, E., Hartig, J., Weyland, U., Nauerth, A., Möllers, M., Rechenbach, S., Simon, J. \& Worofka, I. (2016). Competencies in geriatric nursing: empirical evidence from a computer based large scale assessment calibration study. Vocations and Learning, 9(2), 185-206. 
Kultusministerkonferenz (KMK). (2018). Ländergemeinsame inhaltliche Anforderungen für die Fachwissenschaften in der Lehrerbildung. Online: https://www. kmk.org/fileadmin/Dateien/veroeffentlichungen_beschluesse/2008/2008_10_16Fachprofile-Lehrerbildung.pdf (10.12.2018).

Meyer, R. (2010): Professionalisierung und Professionalität für Tätigkeiten in der Berufsbildung. In K. Büchter (Hrsg.), Enzyklopädie Erziehungswissenschaft Online, Fachgebiet Berufs- und Wirtschaftspädagogik, (S. 1-19). Weinheim, München: Beltz Juventa. Online: https://www.ifbe.uni-hannover.de/fileadmin /ifbe/meyer/Meyer_Professionalisierung_und_Professionalitaet_fuer_Taetigkeite n_in_der_Berufsbildung_2010_1_.pdf(02.11.2018).

Mieg, H. (2016). Profession: Begriff, Merkmale, gesellschaftliche Bedeutung. In M. Dick, W. Marotzki \& H. Mieg (Hrsg.), Handbuch Professionsentwicklung, (S. 27-40). Bad Heilbrunn: Klinkhardt.

Ortenburger, A. (2016). Lehrer und Lehrerinnen. In M. Dick, W. Marotzki \& H. Mieg (Hrsg.), Handbuch Professionsentwicklung, (S. 559-566). Bad Heilbrunn: Klinkhardt.

Reiber, K. (2018). Pflegepädagogik - eine Etappe auf dem Weg der Akademisierung und Professionalisierung der Lehrtätigkeit an pflegeberuflichen Schulen? In M. Friese (Hrsg.), Reformprojekt Care Work. Professionalisierung der beruflichen und akademischen Ausbildung, (S. 61-72). Bielefeld: wbv.

Reiber, K., Weyland, U. \& Zoyke, A. (2017). Herausforderungen und Perspektiven für die Gesundheitsberufe aus der Sicht der Berufsbildungsforschung. In U. Weyland \& K. Reiber (Hrsg.), Entwicklungen und Perspektiven in den Gesundheitsberufen - aktuelle Handlungs- und Forschungsfelder, (S. 9-27). Bielefeld: Bertelsmann.

Reiber, K., Winter, M. H.-J. \& Mosbacher-Strumpf, S. (Hrsg.). (2015). Berufseinstieg in die Pflegepädagogik. Eine empirische Analyse von beruflichem Verbleib und Anforderungen. Lage: Jacobs.

Reiber, K. (2008). Zum Stand der Pflegelehrerbildung - Deutsche Verhältnisse in europäischer Perspektive. In C. Bischoff-Wanner \& K. Reiber (Hrsg.), Lehrerbildung in der Pflege. Standortbestimmung, Perspektiven und Empfehlungen vor dem Hintergrund der Studienreformen, (S. 41-63). Weinheim, München: Beltz Juventa.

Seltrecht, A. (2016). Pflegeberufe. In M. Dick, W. Marotzki \& H. Mieg (Hrsg.), Handbuch Professionsentwicklung, (S. 499-511). Bad Heilbrunn: Klinkhardt.

Seltrecht, A. (2015). Der ,doppelte Fallbezug“ - Herausforderungen in der Lehramtsausbildung in der beruflichen Fachrichtung Gesundheit und Pflege. In K. Jenewein, \& H. Henning (Hrsg.), Neue Handlungsansätze für die Lernorte im Lehramt an berufsbildenden Schulen, (S. 209-227). Bielefeld: Bertelsmann.

Simon, J. (2018). Pflegewissenschaftliche Erkenntnisse in der Unterrichtsplanung. Eine empirische Untersuchung. Unveröff. Diss. a. d. Univ. Bamberg. Bamberg.

Walter, A. (2018). „Große Theorien - mein kleiner Unterricht“, berufsbegleitend Studierende zwischen zwei Welten. In E. Ertl-Schmuck \& J. Hänel (Hrsg.), Passagen pflegedidaktischer Arbeit an der Schnittstelle von Hochschule und Schulpraxis, (S. 90-115). Weinheim: Beltz Juventa. 
Wanner, B. (1993). Lehrer zweiter Klasse? Historische Begründungen und Perspektiven der Qualifizierung von Lehrerinnen und Lehrern der Pflege. Frankfurt am Main u. a.: Peter Lang, 2. Auflage, überarbeitet und erweitert von Claudia Bischoff.

Weyland, U. \& Kaufhold, M. (2017). Qualifizierung des betrieblichen Bildungspersonals in der Pflege. Berufsbildung in Wissenschaft und Praxis, 46(1), 30-33.

Weyland, U. \& Reiber, K. (2013). Lehrer/-innen-Bildung für die berufliche Fachrichtung Pflege in hochschuldidaktischer Perspektive. In U. Faßhauer, B. Fürstenau \& E. Wuttke (Hrsg.), Jahrbuch der berufs- und wirtschaftspädagogischen Forschung 2013, (S. 189-202). Opladen u. a.: Barbara Budrich.

Wittmann, E., Kaspar, R. \& Döring, O. (2017). Berufsfachliche Handlungskompetenz in der unmittelbar klientenbezogenen Pflege: eindimensional oder mehrdimensional? In U. Weyland \& K. Reiber (Hrsg.), Entwicklungen und Perspektiven in den Gesundheitsberufen - aktuelle Handlungs- und Forschungsfelder, (S. 185204). Bielefeld: Bertelsmann.

Wittmann, E. \& Flemming, D. (2018). Domänenspezifische IT-Grundausbildung für angehende Lehrkräfte im Bereich Pflege und Gesundheit. Tagung an der Fachhochschule Fürth zum Erfahrungsaustausch zum Digitalen Campus Bayern. 19.04.2018. Fürth.

Wittmann, E., Weyland, U., Nauerth, A., Döring, O., Rechenbach. S., Simon, J. \& Worofka, I. (2014). Kompetenzerfassung in der Pflege älterer Menschen - Theoretische und domänenspezifische Anforderungen der Aufgabenmodellierung. In J. Seifried, U. Faßhauer \& S. Seeber (Hrsg.), Jahrbuch Berufs- und Wirtschaftspädagogik 2014, (S. 53-66). Opladen u.a.: Budrich. 


\title{
Innere Differenzierung im Unterricht mit jugendlichen Asylsuchenden und Geflüchteten aus der Sicht von Lehrpersonen
}

\author{
Tobias Kärner, Hannes Reinke, Anja Frim und Karin Heinrichs
}

\section{Ausgangslage und Zielstellung}

Im Zuge anhaltender Flucht- und Migrationsbewegungen aus unterschiedlichen Regionen der Erde finden auch zahlreiche Jugendliche und junge Erwachsene den Weg nach Deutschland und somit in das deutsche Bildungssystem. Im Alter von 16 bis 25 Jahren stellen sie dabei einen Anteil von etwa 20 bis $30 \%$ der Asylerstanträge in den vergangenen Jahren und repräsentieren somit eine der größten Altersgruppen (BAMF 2018). Im Sinne einer erfolgreichen Integration gilt es daher vor allem auch bildungspolitisch, den damit einhergehenden Herausforderungen zu begegnen. Lange Zeit standen junge Asylsuchende und Geflüchtete $(\mathrm{jAuG})$ hierzulande nicht im Fokus entsprechender Maßnahmen. Laut Berthold (2014) liegt dies u. a. daran, dass es sich bei ihnen bisher um eine relativ kleine Gruppe im Vergleich zur Gesamtzahl aller Schüler*innen (SuS) in Deutschland handelt. Doch spätestens die veränderte Situation seit der Fluchtwelle im Jahr 2015 verdeutlicht die Notwendigkeit des Umdenkens. In diesem Kontext gilt es auch zu beachten, dass der Anteil der SuS mit Migrationshintergrund bereits vor Jahrzehnten angestiegen ist. Beispielsweise brachte der Mikrozensus aus dem Jahr 2013 hervor, dass beinahe 30 \% der SuS in Deutschland einen Migrationshintergrund haben (StBA 2014). In Abhängigkeit vom Einzugsgebiet stellen sie, gerade in jüngeren Jahrgängen, sogar eine Mehrheit der Schülerschaft dar (Leiprecht \& Steinbach 2015). Dies zeigt einerseits, dass es sich bei SuS mit Migrationshintergrund keineswegs um eine vernachlässigbare Minderheit handelt. Andererseits weisen die Ergebnisse internationaler Vergleichsstudien, wie beispielsweise PISA (Baumert et al. 2001), auf eine Bildungsbenachteiligung der genannten Personengruppe hin. Vergleichbare Ergebnisse können nach Behrensen und Westphal (2009) auch für $\mathrm{jAuG}$ angenommen werden.

Es ist zu berücksichtigen, dass sich Klassen mit Geflüchteten hinsichtlich ihrer Zusammensetzung durch einen hohen Grad an Heterogenität auszeichnen, da sich neben interindividuellen Unterschieden in Herkunftsländern, Konfessionen und Muttersprachen vor allem große Differenzen in den jeweiligen schulischen Vorkenntnissen und im Sprachniveau zeigen (z. B. Maué, Schumann \& Diehl 2018). Die individuellen Lernvoraussetzungen streuen hierbei 
breit: von Lernenden, welchen eine grundlegende Alphabetisierung fehlt bis zu SuS, welche in ihrem Herkunftsland bereits eine landesspezifisch gute schulische Ausbildung erhalten haben. Der Umgang mit leistungsbezogener, kultureller, religiöser und sprachlicher Heterogenität im Klassenzimmer stellt für die eingesetzten Lehrpersonen eine große Herausforderung dar (Kärner, Feldmann, Heinrichs, Neubauer \& Sembill 2016). Die Lehrkräfte sind mit der Aufgabe konfrontiert, möglichst alle Lernenden zu Handlungsfähigkeit und Berufsreife zu führen und gleichzeitig den spezifischen Bedürfnissen des/der Einzelnen gerecht zu werden (Witte \& Harden 2010). Um mit der betreffenden Schülerschaft umzugehen, wird der Einsatz von Methoden und Maßnahmen der inneren Differenzierung (i. D.) gefordert (z. B. Zoyke 2017). So soll den individuellen Bedürfnissen der Lernenden möglichst gut nachgekommen und somit zu einer gelingenden beruflichen, schulischen und gesellschaftlichen Integration beigetragen werden (vgl. Heinrichs et al. 2016). Welche konkreten Maßnahmen der Binnendifferenzierung im Unterricht mit jAuG jedoch als sinnvoll und praktikabel erscheinen und welche Kompetenzen und Eigenschaften zur Umsetzung entsprechender Maßnahmen aus Sicht der Lehrenden als notwendig und relevant eingeschätzt werden, erscheint bislang nicht hinlänglich erforscht zu sein. Hier setzt der vorliegende Beitrag an. Im Rahmen einer explorativen Interviewstudie soll den folgenden Forschungsfragen nachgegangen werden:

1. Auf welche unterrichtsbezogenen Aspekte zielen die im Rahmen der Beschulung von jAuG eingesetzten Methoden der i. D. ab und welche Schüler*innenmerkmale werden als Differenzierungskriterien herangezogen?

2. Welche Grenzen bzw. Barrieren sehen Lehrkräfte bei der Umsetzung von Methoden der i. D. in sog. Flüchtlingsklassen?

3. Inwiefern halten sich die Lehrkräfte für fähig bzw. ausreichend ausgebildet, Methoden der i. D. im Unterricht mit jAuG adäquat anzuwenden und welche Fortbildungsmaßnahmen sehen sie als erforderlich an?

Es wurden insgesamt 16 Lehrpersonen, welche in Flüchtlingsklassen in der Sekundarstufe unterrichten, mittels Leitfrageninterviews befragt. Die Aussagen wurden anschließend inhaltsanalytisch ausgewertet. Nachfolgend werden generelle bildungspolitische Bedingungen zur Beschulung von $\mathrm{jAuG}$ im deutschen Bildungssystem sowie Potenziale und Herausforderungen der i. D. im Rahmen der Flüchtlingsbeschulung diskutiert. 


\section{Hintergrund}

\subsection{Jugendliche Asylsuchende und Geflüchtete im deutschen Bildungssystem}

Seit dem Jahr 2010 haben nahezu zwei Millionen Menschen Asyl in Deutschland gesucht, wobei die meisten Anträge im Zeitraum von 2015 bis 2017 gestellt wurden. Eine der zahlenmäßig stärksten Gruppen unter den Erstantragsstellern stellen Jugendliche und junge Erwachsene im Alter von 16 bis 25 Jahren dar, die etwa 20 bis $30 \%$ der jährlichen Kohorten ausmachen und als besonders (aus-)bildungsrelevant gelten (BAMF 2018). Jäger (2002) kritisiert in diesem Zusammenhang, dass die Mehrzahl der Zielländer der in verschiedenen Abkommen festgehaltenen Aufgabe, Geflüchteten am gesellschaftlichen und wirtschaftlichen Leben teilhaben zu lassen, nicht gerecht werden und keine angemessene Förderung umsetzen. Der Stellenwert von Bildungsangeboten werde im Fall von jAuG häufig als nachrangig betrachtet (ebd.), obwohl Bildung für eine erfolgreiche Integration maßgeblich ist (Berthold 2014).

In Deutschland ist man um eine Umsetzung entsprechender internationaler Abkommen und Verträge bemüht (z. B. UNGA 1948). JAuG werden in zahlreichen, bundeslandspezifisch konzipierten Initiativen und Maßnahmen schulisch qualifiziert und auf eine Ausbildung vorbereitet. Ziel der verschiedenen Maßnahmen ist es hierbei, durch Bildung und Qualifikation eine gelingende Integration in die deutsche Gesellschaft zu ermöglichen (ISB 2017). In Bayern bspw. wurden großflächig sogenannte Berufsintegrationsklassen implementiert (für eine aktuelle Analyse zu jungen Geflüchteten in der Berufsvorbereitung in Baden-Württemberg siehe Maué et al. 2018). Asylsuchende und Geflüchtete haben die Möglichkeit vom 17ten bis zum 25sten Lebensjahr die Berufsschule zu besuchen und sich wichtige Kompetenzen ,für eine erfolgreiche Berufsausbildung und gelingende Integration“ (ISB 2017, 4) anzueignen. Das Konzept der Berufsintegrationsklassen sieht insgesamt zwei jeweils einjährige Phasen der Beschulung vor. In der Vorklasse liegt der Fokus v. a. auf der Alphabetisierung und dem Spracherwerb. Im zweiten Jahr werden neben der weiterführenden Sprachförderung v. a. berufsvorbereitende Aspekte thematisiert, welche optimaler Weise von geeigneten Betriebspraktika zur Berufsorientierung begleitet werden (hierzu Reinke, Kärner \& Heinrichs 2018). Der Besuch der zwei Schuljahre schließt mit dem Erwerb der Berechtigungen eines Mittelschulabschlusses ab (ISB 2017). 


\subsection{Innere Differenzierung im Rahmen der Flüchtlingsbeschulung}

\subsubsection{Begriffsverständnis}

Da es sich bei den $\mathrm{jAuG}$ in den betreffenden Klassen um eine als heterogen wahrgenommene Gruppe handelt (z. B. Heinrichs et al. 2016; Kärner et al. 2016; Schumann, Kärner \& Maué 2018), macht dies in besonderer Weise eine differenzierte und auf die Lernenden angepasste Unterrichtsgestaltung erforderlich. Von i. D. bzw. Binnendifferenzierung spricht man im schulischen Kontext, wenn es um „Unterscheidung, Verfeinerung, Abstufung und Aufteilung der Lerninhalte" (Paradies \& Linser 2013, 10) sowie um die Adaption der Lehrmethode, des Lerngegenstands und des Medieneinsatzes an die individuellen Lernvoraussetzungen der SuS geht. Bönsch (2016) definiert i. D. als alle Maßnahmen, „die innerhalb fester Lerngruppen (Klassen) Lernwege, Lernaufgaben, Lernansprüche, Lernzeiten und Lernhilfen für einzelne Lerner oder mindestens für Teilgruppen variabel und damit unterschiedlich gestalten." (208f.). Hierbei wird das Ziel einer möglichst individualisierten Förderung angestrebt (Kranert \& Stein, in Druck). Die i. D. ist abzugrenzen von der äußeren Differenzierung, bei welcher anhand von Selektionsmaßnahmen (z. B. Jahrgangsklassen, Schulformen) versucht wird, möglichst homogene Personengruppen zu bilden, welche räumlich getrennt voneinander unterrichtet werden (Scholz 2010). Für eine erfolgreiche Integration von Maßnahmen der i. D. in den schulischen Unterricht sind nach Klafki und Stöcker (2007) Variationen didaktischer Entscheidungsfelder hinsichtlich Aufgabenstellung (z. B. Stoffumfang, Zeitaufwand), Unterrichtsmethoden (z. B. Stationenlernen, Freiarbeit, Projektarbeit), Medien (z. B. Texte, Bilder, Computer), Sozialformen (z. B. Gruppenarbeit, Einzelarbeit) und dem Grad der Lehrerhilfe (z. B. Grad der Strukturierungshilfe) denkbar.

\subsubsection{Herausforderungen bei der Förderung von SuS mit Fluchthintergrund}

Binnendifferenzierungsmaßnahmen mit dem Zweck der individuellen Förderung werden als zielführend betrachtet, um SuS mit Migrations- bzw. Fluchthintergrund adäquat zu unterstützen (Behrensen \& Westphal 2009). Gerade in Deutschland spielt die Herkunft von Kindern und Jugendlichen für den Bildungserfolg eine zentrale Rolle. Den Ergebnissen der PISA-Studie(n) zufolge, schneiden SuS mit Migrations- bzw. Fluchthintergrund deutlich schlechter ab als SuS ohne entsprechenden Hintergrund (Kauffmann, Knapp, Novotny \& Schoch 2002). Dies hat wiederum zur Folge, dass Migranten in Förder- und Hauptschulen überrepräsentiert sind (Leiprecht \& Steinbach 2015) und hinsichtlich einer förderlichen Unterrichtsgestaltung besonderer Aufmerksamkeit bedürfen. Betrachtet man die Befundlage zur Wirksamkeit differenzierender 
Unterrichtsmaßnahmen, zeigt sich allerdings ein eher ernüchterndes Bild (vgl. Beck et al. 2008). Erklärungsversuche für die geringen Effekte von i. D. bzw. von adaptivem Unterricht setzen meist bei den hohen und komplexen Anforderungen an die Lehrpersonen an, die eine vollständige Umsetzung des Konzepts mit sich bringt. Weinert (1997) zufolge grenzt die Umsetzung an ein „wahrhaft herkulische[s] pädagogische[s] Problem“ (50), da die Gelingensbedingungen von der Motivation und dem Interesse der zu fördernden SuS bis hin zu strukturellen Bedingungen, geeigneten Räumlichkeiten, zeitlichen Ressourcen, flexiblen Budgets sowie dem Ausbildungsstand des Lehrpersonal reichen. Die gängige Praxis zeigt jedoch, dass sich diese weitreichenden Gelingensbedingungen in der Flüchtlingsbeschulung nur schwer umsetzen lassen. Barth und Guerrero Meneses (2012) kommen im Rahmen ihrer Interviewstudie zu dem Ergebnis, dass jAuG innerhalb der bestehenden Struktur von Förder- und Übergangsklassen nur sehr schwer entsprechend ihrer Fähigkeiten unterstützt werden können.

\subsubsection{Relevante Dimensionen des Lehrer*innenhandelns}

In der Diskussion um einen professionellen, ressourcen- und chancenorientierten Umgang mit Heterogenität wird insbesondere den Lehrkräften vorgeworfen, dass diese die Vielfalt ihrer SuS eher als Störgröße denn als Normalfall betrachten (Leiprecht \& Lutz 2015). Somit wird der Eindruck vermittelt, dass die Potenziale der i. D. in erster Linie deshalb eingeschränkt sind, weil die Vorbehalte (z. B. bezogen auf den zeitlichen und organisationalen Aufwand) vieler Pädagogen*innen bzw. Lehrender einer Umsetzung vorhandener Ideen entgegenstehen (Wischer \& Trautmann 2012). Die Forderung lautet demnach, dass Heterogenität von den Lehrenden idealerweise als Chance und nicht als Belastung verstanden werden soll (vgl. Terhart 2015). Wischer (2007) hingegen warnt davor, die bisherigen Defizite der Unterrichtspraxis im Umgang mit Vielfalt vorwiegend als eine Frage des Wollens zu interpretieren und dabei die Frage des Könnens außer Acht zu lassen. Wenn sich Handlungsempfehlungen ausschließlich auf die didaktisch-methodische Unterrichtsgestaltung konzentrieren, bleibt die Frage offen, inwiefern die notwendigen Voraussetzungen für deren Umsetzung - z. B. durch strukturelle Bedingungen oder durch entsprechende Ausbildungsinhalte - gegeben sind (siehe auch Trautmann \& Wischer 2013). In diesem Zusammenhang können die Befunde von Roeder (1997) angeführt werden. Diese weisen darauf hin, dass Binnendifferenzierung und individuelle Förderung von den Befragten durchaus als notwendige und wichtige Ziele wahrgenommen werden. Die befragten Lehrpersonen stehen dem Thema generell positiv gegenüber. Allerdings setzt nur eine Minderheit die erforderlichen Maßnahmen tatsächlich um. 
Da sich nach Wischer (2007) die Anforderungen an das konkrete Lehrerhandeln als sehr voraussetzungsreich darstellen, sind neben den Rahmenbedingungen auch die notwendigen Lehrer*innenkompetenzen genauer zu betrachten. Im Zuge der Diskussion bezüglich innerer Differenzierungsmaßnahmen wird hierbei auf das Konzept der adaptiven Lehrkompetenz verwiesen (z. B. Beck et al. 2008; Wischer 2007; Wischer \& Trautmann 2012). Adaptive Lehrkompetenz setzt sich hierbei aus vier Dimensionen zusammen: (1) Sachkompetenz (Beherrschen von Anforderungen des Unterrichtsinhalts), (2) diagnostischer Kompetenz (korrekte Beurteilung von Kenntnisstand, Lernfortschritten und Leistungsproblemen einzelner SuS), (3) didaktischer Kompetenz (Einsetzen unterschiedlicher Unterrichtsformen zur Erreichung unterschiedlicher pädagogischer Ziele) sowie (4) Klassenführungskompetenz (pädagogische Maßnahmen zur Steuerung, Führung und Begleitung der SuS einer Klasse) (Beck et al. 2008). Gruehn (2000) stellt vor allem die hohen Anforderungen an die methodischen und diagnostischen Kompetenzen von Lehrenden heraus. Im Bereich der didaktischen Kompetenz zeigen Forschungsergebnisse bereits der 1990er Jahre, dass Lehrpersonen eine Vielfalt von Methoden im Unterricht einsetzen (z. B. Dichanz \& Schwittmann 1999). Allerdings zeigen die bestehenden Befunde auch auf, dass erforderliche Maßnahmen nicht zwingend im Sinne der i. D. umgesetzt werden. Wischer (2007) kommt deshalb zu dem kritischen Fazit, dass sich Lehrkräfte zwar um einen abwechslungsreichen, schüleraktiven Unterricht bemühen, dabei jedoch selten differenzierte Lernwege eröffnen. Die referenzierten Befunde zeigen einerseits eine positive Einstellung der Lehrpersonen zur Thematik der i. D., andererseits jedoch eine mangelnde unterrichtspraktische Umsetzung auf. Somit scheint es sinnvoll, bereits im Rahmen konzeptioneller Überlegungen verstärkt auf die Frage des Könnens einzugehen, um die Diskrepanz zwischen der vorhandenen Einsicht über die Notwendigkeit innerer Differenzierungsmaßnahmen und der mangelnden schulpraktischen Umsetzung zu überwinden.

\section{Stichprobe und Untersuchungsmethode}

In der vorliegenden Untersuchung sollen die Sichtweisen der Lehrkräfte zu Methoden der i. D. sowie die spezifischen Bedarfe bzw. Bedingungen in den Übergangsklassen für jAuG exploriert werden. Dazu wurden Lehrpersonen aus der Sekundarstufe (Mittelschule, Berufsschule; Bayern) rekrutiert (Frim 2016). Insgesamt wurden 16 Personen (50 \% männlich) mit einem durchschnittlichen Alter von 41,7 Jahren (Min. = 31, Max. =64) befragt. 14 Personen haben ein Lehramtsstudium absolviert, zwei weitere Personen haben einen Abschluss im Fach „Deutsch als Zweitsprache“ (DaZ). Zudem besitzen elf der 
befragten Lehrkräfte eine DaZ-Zusatzqualifikation. Die Erfahrung im Unterrichten von Asylsuchenden und Geflüchteten bzw. Migranten liegt durchschnittlich bei 7,4 Jahren (Min. = 1, Max. =25). Die 16 Lehrkräfte wurden einzeln und persönlich in halbstrukturierten Interviews befragt. Im Anschluss an die Datenerhebung wurden die Audiodaten transkribiert und mittels strukturierender Inhaltsanalyse auf Grundlage eines deduktiv-induktiven Kategorienschemas ausgewertet. ${ }^{1}$

\section{Ergebnisse}

\subsection{Unterrichtsbezogene Ansatzpunkte innerer Differenzierung}

Hinsichtlich der Frage, auf welche unterrichtsbezogenen Aspekte die im Rahmen der Beschulung von jAuG eingesetzten Methoden der i. D. abzielen, finden sich die häufigsten Nennungen zur Differenzierung anhand der Aufgabenstellung (z. B. Variation des Schwierigkeitsgrads) (15). Jeweils elf Nennungen entfallen auf die seitens der Lehrkraft getätigte unmittelbare individuelle Unterstützung bzw. Hilfestellung („Lehrerhilfe“) und auf die Sozialform (z. B. Gruppenarbeiten, Einzelarbeit). Darüber hinaus wurden Medien (z. B. Lernkarten, haptische Materialien) (5) und didaktische Methoden (z. B. Lerntheken) (2) als weitere Ansatzpunkte hervorgehoben (Abb. 1).

\section{Abb. 1: Unterrichtsbezogene Ansatzpunkte innerer Differenzierung}

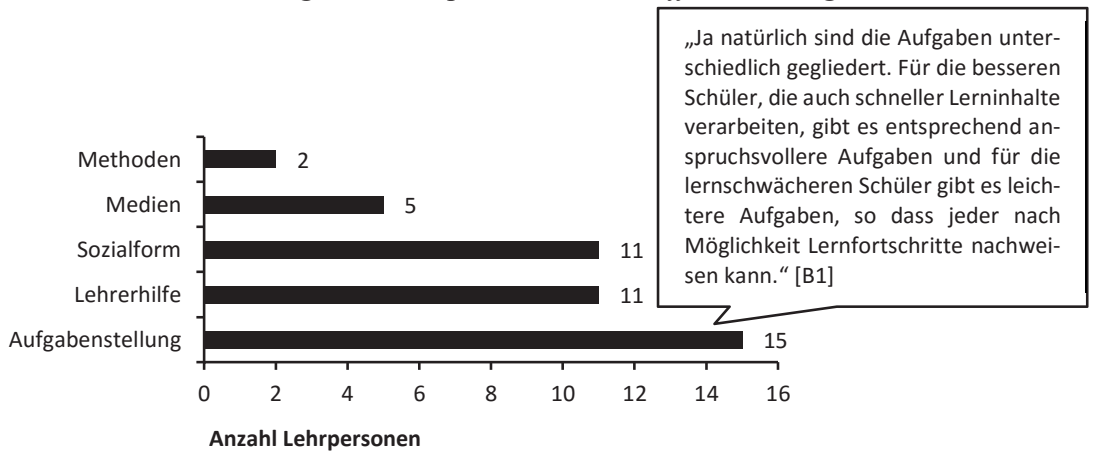

Quelle: eigene Darstellung

1 Intracoderreliabilität: Cohens Kappa-Werte von 0,85 (auf Basis von 61 doppelt kodierten Sinneinheiten) bzw. von 0,86 (74 doppelt kodierte Sinneinheiten). Intercoderreliabilität: Cohens Kappa-Werte von 0,76 (44 doppelt kodierte Sinneinheiten) bzw. 0,73 (70 doppelt kodierte Sinneinheiten). 


\subsection{Schüler*innenmerkmale als Differenzierungskriterien}

Bezogen auf die Frage, welche Schüler*innenmerkmale von den Lehrpersonen als Differenzierungskriterien herangezogen werden, orientieren sich alle befragten Lehrpersonen an dem Leistungsvermögen der Lernenden, welches meist mit den vermuteten kognitiven Kapazitäten (i. d. Grundintelligenz) gleichgesetzt wird. Die Hälfte der Teilnehmer*innen nannte das Sprachniveau in der deutschen Sprache als weiteres Differenzierungsmerkmal, gefolgt von soziodemografischen Merkmalen (z. B. Herkunft, Schichtzugehörigkeit, Sprache). Auch wurden das Interesse der Lernenden (z. B. bzgl. individueller Berufswünsche und -vorstellungen), deren Alter und das Geschlecht als mögliche Differenzierungsmerkmale genannt (Abb. 2).

Abb. 2: Schüler*innenmerkmale als Differenzierungskriterien

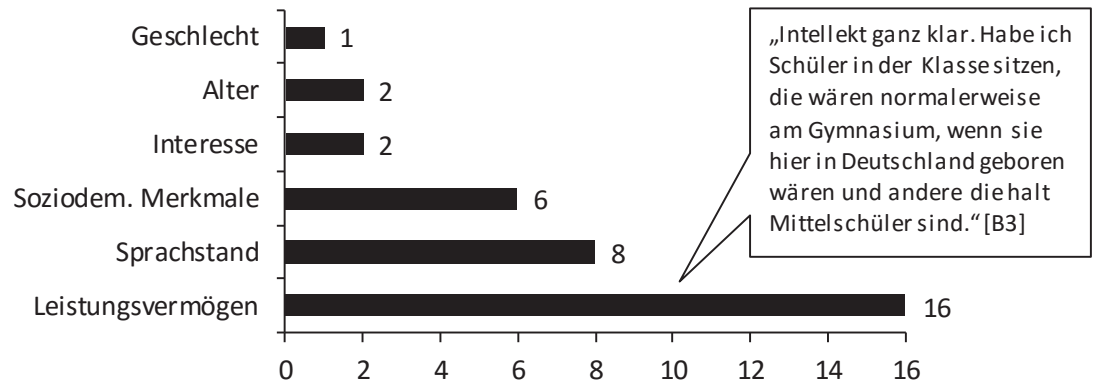

Anzahl Lehrpersonen

Quelle: eigene Darstellung

\subsection{Wahrgenommene Grenzen des Einsatzes innerer Differenzierungsmaßnahmen}

Auf die Frage nach wahrgenommenen Grenzen des Einsatzes innerer Differenzierungsmaßnahmen wurde eine ganze Bandbreite an möglichen Problemfeldern genannt. Insbesondere der zeitliche und organisatorische Mehraufwand für die Planung und den Einsatz von Binnendifferenzierungsmaßnahmen wird als sehr hoch eingeschätzt (13). Weiterhin wurden ungünstige Schüler*innenvoraussetzungen (z. B. geringe Vorkenntnisse und Fertigkeiten, psychische Beeinträchtigungen) und organisatorische Rahmenbedingungen (z. B. fehlende Räumlichkeiten und Lehr-/Lernmaterialien) von jeweils zwölf Lehrpersonen genannt. Auch sehen die Befragten in der „Heterogenität“ der Schülerschaft (11; z. B. bzgl. individueller Lernvoraussetzungen), dem Mangel an 
Lehrpersonal (10) sowie in großen Klassen (10) zusätzliche Problemfelder. Die eigene unzureichende Ausbildung im Bereich der i. D. (7), Sprachbarrieren (7), kulturelle Konflikte zwischen den Lernenden (5) sowie eine seitens der SuS wahrgenommene Ungleichbehandlung (3; z. B. aufgrund unterschiedlicher Zuwendungsgrade durch die Lehrperson) werden als weitere Grenzen des Einsatzes innerer Differenzierungsmaßnahmen genannt (Abb. 3).

\section{Abb. 3: Wahrgenommene Grenzen des Einsatzes innerer Differenzierungsmaßnahmen}

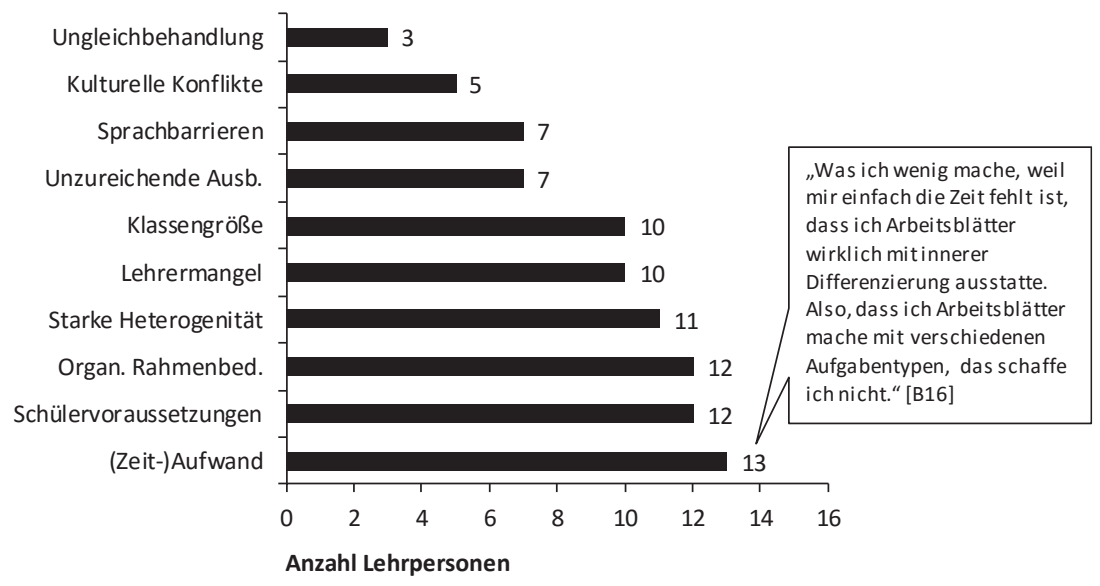

Quelle: eigene Darstellung

\subsection{Als relevant erachtete Lehrer*innenkompetenzen und -eigenschaften}

Auf die Frage nach Lehrer*innenkompetenzen und -eigenschaften, die für die Realisierung des Konzepts der i. D. im Flüchtlingsunterricht nach Ansicht der Befragten relevant sind, erwähnten zwölf von 16 Lehrpersonen interkulturelle und soziale Kompetenzen (z. B. Wissen bzgl. der Herkunftsländer), gefolgt von didaktischen (9; z. B. angemessene Aufbereitung der Lernmaterialien), diagnostischen (8; z. B. angemessene Lernstandserfassung) und Organisationskompetenzen (8; z. B. zeitliche Strukturierung des Unterrichts). Weiterhin nannten sechs der Befragten Geduld als eine wichtige Eigenschaft (z. B. aufgrund unterschiedlicher Lerngeschwindigkeiten). Jeweils zwei Lehrpersonen betonten Klassenführungs- (z. B. Anleiten von Lerngruppen) und Sachkompetenz (z. B. Fachkenntnisse bzgl. der behandelten Inhalte) (Abb. 4). 
Abb. 4: Als relevant erachtete Lehrer*innenkompetenzen und-eigenschaften

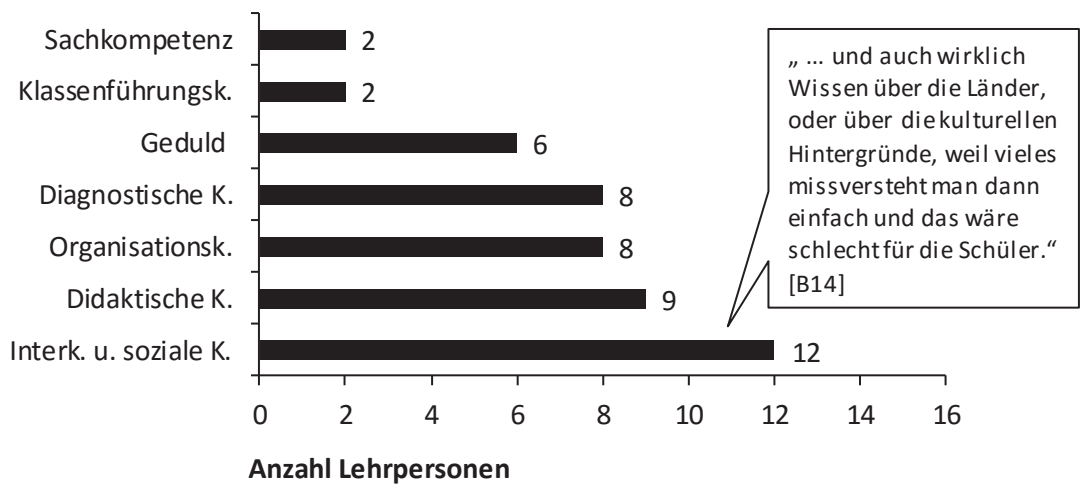

Quelle: eigene Darstellung

\subsection{Thematisierung in der Lehrer*innenbildung und Fort- und Weiterbildungsbedarfe}

Hinsichtlich der Frage, inwiefern sich die Lehrkräfte für fähig bzw. ausreichend ausgebildet halten, Methoden der i. D. im Unterricht mit jAuG adäquat anzuwenden, erachteten zwölf der befragten Lehrpersonen die Behandlung von Binnendifferenzierungsmethoden in ihrem Studium als lückenhaft und nur zwei Lehrpersonen haben den Eindruck, dass dieses Thema in ihrem Studium in ausreichendem Maße behandelt wurde. Neun von 14 Personen (zwei der Befragten haben kein Referendariat absolviert) sehen die Thematisierung von Binnendifferenzierungsmaßnahmen im Referendariat als ausreichend behandelt an. Fünf Personen dagegen vertreten die Meinung, dass in der zweiten Phase der Lehrer*innenbildung das Thema nur lückenhaft behandelt werde.

Nach notwendigen Fort- und Weiterbildungsthemen gefragt, machten zwölf Lehrpersonen deutlich, dass sie im didaktischen Bereich hinsichtlich konkreter Methoden und Instrumente der Binnendifferenzierung Bedarf sehen. Eine Lehrperson sieht für sich, trotz vorhandener DaZ-Qualifikation, Bedarf bezüglich der Vermittlung einer grundlegenden Alphabetisierung, eine andere Lehrkraft erachtet für sich eine Fortbildung im Bereich der Didaktik des Deutschen als Zweitsprache als sinnvoll und eine weitere Lehrperson sah die Notwendigkeit in Bezug auf Diagnostikverfahren hinsichtlich des individuellen Leistungsvermögens der Lernenden. 


\section{Schlussteil}

\subsection{Limitationen der Studie}

Limitierend ist bzgl. der Interviewstudie anzumerken, dass diese lediglich einen ersten explorativen Zugang zum behandelten Themenschwerpunkt darstellt und aufgrund der qualitativen Methodik sowie der kleinen Stichprobe keine (statistische) Prüfung von Hypothesen zulässt. Um insbesondere hinsichtlich der Wirkung binnendifferenzierender Maßnahmen auf lern-, leistungs- und entwicklungsrelevante Indikatoren der betrachteten Klientel belastbare Aussagen treffen zu können, sind zukünftig entsprechende experimentelle Versuchsanordnungen unumgänglich. Hierfür kann die vorliegende Studie jedoch einen orientierungsgebenden Ausgangspunkt darstellen. Weiterhin ist anzumerken, dass die Datenerhebung bereits im Jahr 2016 stattfand, was angesichts des sich schnell entwickelnden und verändernden Gegenstandsbereichs der Beschulung jAuG ebenfalls einen limitierenden Faktor darstellt.

\subsection{Von wahrgenommenen Barrieren zu Entwicklungschancen}

Das Wollen bzgl. des Einsatzes innerer Differenzierungsmaßnahmen im Unterricht mit jAuG im Sinne von Wischer (2007) scheint nach den Aussagen der von uns befragten Lehrpersonen gegeben zu sein. Auch werden bereits unterschiedliche Maßnahmen und Methoden in den Unterricht integriert, was jedoch in der schulischen Praxis an unterschiedlichen Stellen an seine Grenzen stößt - die Frage des Könnens i. S. v. Wischer (2007) ist demnach vor dem Hintergrund der gefundenen Ergebnisse zu diskutieren.

Die Etablierung individueller Förderung setzt jedoch entsprechende Rahmenbedingungen voraus. Die identifizierten Barrieren, welche nach den Aussagen der Lehrkräfte eine Umsetzung von Binnendifferenzierungsmaßnahmen im Unterrichtsalltag erschweren, reichen von allgemeinen Rahmenbedingungen bis zur Ebene der beteiligten Personen. Hierbei wird deutlich, dass eine erfolgreiche Etablierung von Beschulungsmaßnahmen für $\mathrm{jAuG}$ vermutlich nur über eine sehr enge Verzahnung von äußeren und inneren Differenzierungsmaßnahmen gelingen kann. Dies zeigte sich auch in unserer Untersuchung, wobei auf der Ebene der Lehrpersonen besonders häufig die Faktoren (Zeit-)Aufwand, organisatorische Rahmenbedingungen und Schüler*innenvoraussetzungen als kritische Punkte genannt wurden (siehe auch Heinrichs et al. 2016; Heinrichs, Reinke \& Feldmann 2018). Hinzu kommen der sog. „Lehrermangel", die Klassengrößen und spezifische Grenzen im Bereich der Flüchtlingsbeschulung wie z. B. die „starke Heterogenität“ der Schülerschaft sowie Sprachbarrieren. Zumindest bzgl. des letztgenannten Punktes wird versucht, 
mit Hilfe des Unterrichtsprinzips „Berufssprache Deutsch“ und bereitgestellter Materialien zur sprachsensiblen Unterrichtsgestaltung, Unterstützungsangebote für Lehrpersonen bereitzustellen (hierzu auch Terrasi-Haufe, Hofmann \& Sogl 2018). Die Ausführlichkeit der genannten Hindernisse stützt das Bild, nach dem es sich aus Sicht der Lehrkräfte bei der Binnendifferenzierung um ein sehr voraussetzungsreiches Konzept handelt. Die seitens der Lehrpersonen wahrgenommene Überforderung bei der praktischen Umsetzung verwundert also nicht.

Eine weitere Grenze, die im Rahmen der Untersuchung zur Sprache kam, ist die als unzureichend empfundene Ausbildung bezüglich der Thematik der inneren Differenzierung. Dabei sehen die Befragten vorwiegend für die erste Phase der Lehramtsausbildung an den Hochschulen starken Nachholbedarf. Zwar gaben die meisten Befragten an, dass das Konzept der Binnendifferenzierung während des Studiums thematisiert wurde, erachten die als berufsalltagsfern wahrgenommenen Ausführungen jedoch nicht für ausreichend und zielführend. Aus diesem Grund ist über eine stärkere Kooperation der Universitäten und der Schulen bereits in der ersten Phase der Lehramtsausbildung nachzudenken, wie dies beispielsweise im Zuge der bayerischen Universitätsschulinitiative verfolgt wird (z. B. Bauer, Blum \& Sembill 2012).

\subsection{Ausblick: Passungsverhältnis zwischen Lernenden und Lernkontext}

Die Ergebnisse zeichnen ein Bild, nach dem alle der 16 befragten Lehrkräfte den Einsatz innerer Differenzierungsmaßnahmen im Unterricht mit jAuG grundsätzlich für notwendig und sinnvoll erachten. Sie sehen sich jedoch angesichts der als ,,sehr heterogen“ wahrgenommenen Schülerschaft vor der Herausforderung, ihren Unterricht adressatengerecht zu gestalten und Lernangebote $\mathrm{zu}$ schaffen, welche allen Lernenden möglichst gut gerecht werden. Hierzu erscheint es nicht ausreichend, gelegentliche, summative Lernstandsdiagnosen zum Zwecke der Notenfindung durchzuführen. Vielmehr müssen die Lehrpersonen, um Lernverläufe nachvollziehen und adäquat begleiten zu können, im Unterrichtsprozess permanent diagnostische Informationen aufnehmen und verarbeiten, was in der unterrichtlichen Praxis i. d. R. beiläufig und routinebasiert geschieht. Die lehrer*innenseitige Verarbeitung von Informationen als Grundlage bedarfsgerechter und situationsangemessener didaktischer Entscheidungen ist hierbei jedoch $u$. a. aufgrund von Informationsflut und -menge sowie beschränkter Beobachtbarkeit schüler- und unterrichtsseitiger Lernindikatoren sehr anfällig für Urteilsfehler und damit einhergehende Fehleinschätzungen (Warwas, Kärner \& Golyszny 2015). Angesichts ,heterogener" Lernvoraussetzungen und -bedingungen sind hierbei neben interindividuellen Unterschieden zwischen Lernenden (z. B. individuelles Vorwissen zu Beginn eines neuen Unterrichtsthemas), ebenso intraindividuelle Veränderungen 
(z. B. zeitpunktbezogene Fachwissensniveaus) sowie Variationen im Zusammenspiel zwischen den Lernenden und dem Lernkontext (z. B. Interaktionen zwischen individuellen Fachwissensniveaus und situativen Aufgabenschwierigkeiten) zu bedenken (Kärner, Warwas \& Schumann 2019). Dieses komplexe Interaktionsgefüge macht es insbesondere vor dem Hintergrund der Fragestellung nach einer möglichst optimalen Passung zwischen Differenzierungsmaßnahmen und schüler*innenseitigen Differenzierungskriterien erforderlich, Lehrpersonen durch Möglichkeiten der gezielten Analyse und Aufbereitung von inhalts- und schülerbezogenen Informationen beim Treffen begründeter pädagogisch-diagnostischer Entscheidungen zu unterstützen, was u. a. durch entsprechende Informationssysteme erreicht werden kann (z. B. Kärner, Fenzl, Warwas \& Schumann 2019; Kärner, Warwas \& Schumann 2019).

\section{Literatur}

BAMF (Bundesamt für Migration und Flüchtlinge). (2018). Aktuelle Zahlen zu Asyl (11/2018). Online: http://www.bamf.de/DE/Infothek/Statistiken/Asylzahlen/AktuelleZahlen/aktuelle-zahlen-asyl-node.html (15.01.2019).

Barth, S. \& Guerrero Meneses, V. (2012). Zugang jugendlicher Asylsuchender zu formellen Bildungssystemen in Deutschland. Zwischen Kompetenzen und strukturellen Problemlagen. Online: https://isis-sozialforschung.de/docs/Bildungszug $\% \mathrm{c} 3 \% \mathrm{a} 4$ ngef\%c3\%bcrAsylsuchende.pdf (06.05.2016).

Bauer, C., Blum, E. \& Sembill, D. (2012). Bamberger Universitätsschulen: Sicherung von Qualität und Quantität der Lehrerbildung in der beruflichen Bildung. Wirtschaft \& Erziehung, 64(7), 218-220.

Baumert, J., Klieme, E., Neubrand, M., Prenzel, M., Schiefele, U., Schneider, W., Stanat, P., Tillmann, K.-J. \& Weiß, M. (Hrsg.). (2001). PISA 2000. Basiskompetenzen von Schülerinnen und Schülern im internationalen Vergleich. Opladen: Leske + Budrich.

Beck, E., Baer, M., Guldimann, T., Bischoff, S., Brühwiler, C., Müller, P., Niedermann, R., Rogalla, M. \& Vogt, F. (Hrsg.). (2008). Adaptive Lehrkompetenz. Analyse und Struktur, Veränderbarkeit und Wirkung handlungssteuernden Lehrerwissens. Münster: Waxmann.

Behrensen, B. \& Westphal, M. (2009). Junge Flüchtlinge - ein blinder Fleck in der Migrations- und Bildungsforschung. Bildung junger Flüchtlinge als Randthema in der migrationspolitischen Diskussion. In L. Krappmann, A. Lob-Hüdepohl, A. Bohmeyer \& S. Kurzke-Maasmeier (Hrsg.), Bildung für junge Flüchtlinge - ein Menschenrecht. Erfahrungen, Grundlagen und Perspektiven, (S. 45-58). Bielefeld: Bertelsmann.

Berthold, T. (2014). In erster Linie Kinder. Flüchtlingskinder in Deutschland. 2014. Online: https://www.unicef.de/blob/56282/fa13c2eefcd41dfca5d89d44c72e72e3* fluechtlingskinder-in-deutschland-unicef-studie-2014-data.pdf (05.05.2016). 
Bönsch, M. (2016). Heterogenität verlangt Differenzierung. Die berufsbildende Schule, $68(6), 208-212$.

Dichanz, H. \& Schwittmann, D. (1999). Das Methodenrepertoire von Lehrern. Eine Unterrichtsvariable im Brennpunkt. In U. Steffens \& T. Bargel (Hrsg.), Lehren und Lernen im offenen Unterricht. Empirische Befunde und kritische Anmerkungen. Beiträge aus dem Arbeitskreis „Qualität der Schule“, (S. 12-17). Wiesbaden: HELP.

Frim, A. (2016). Analysen zur inneren Differenzierung im Bereich Flüchtlingsbeschulung mittels Lehrerbefragung. Unveröffentlichte Masterarbeit an der Professur für Wirtschaftspädagogik (Prof. Dr. K. Heinrichs), Otto-Friedrich-Universität Bamberg.

Gruehn, S. (Hrsg.). (2000). Unterricht und schulisches Lernen. Schüler als Quellen der Unterrichtsbeschreibung. Münster: Waxmann.

Heinrichs, K., Kärner, T., Ziegler, S., Feldmann, A., Reinke, H. \& Neubauer, J. (2016). Die Implementierung neuer Konzepte zur Beschulung von Flüchtlingen und Asylsuchenden. Herausforderungen und Chancen aus organisationstheoretischer Perspektive. Gruppe. Interaktion. Organisation. Zeitschrift für Angewandte Organisationspsychologie, 47(3), 231-241.

Heinrichs, K., Reinke, H. \& Feldmann, A. (2018). Herausforderungen und Chancen in der Beschulung von Geflüchteten in Berufsintegrationsklassen aus Sicht der Schulen und Verwaltung 2015 und heute - eine Zwischenbilanz. Vlb-Akzente, 1/2018, $15-18$.

ISB (Staatsinstitut für Schulqualität und Bildungsforschung). (2017). Lehrplan für die Berufsintegrations- und Sprachintensivklassen. Online: https://www.isb.bayern.de /download/19735/lp_berufsintegrationsklassen_07_2017.pdf (15.01.2019).

Jäger, T. (2002). They don't need no education? Flüchtlinge und Bildung. Zeitschrift für internationale Bildungsforschung und Entwicklungspädagogik, 25(2), 2-9.

Kärner, T., Feldmann, A., Heinrichs, K., Neubauer, J. \& Sembill, D. (2016). Herausforderungen bei der Beschulung von Asylsuchenden und Flüchtlingen im Rahmen von BAF-Klassen an beruflichen Schulen: Deskriptive Befunde aus einer Interviewstudie. Wirtschaft \& Erziehung, 68(2), 58-62.

Kärner, T., Fenzl, H., Warwas, J. \& Schumann, S. (2019). Digitale Systeme zur Unterstützung von Lehrpersonen - Eine kategoriengeleitete Sichtung generischer und anwendungsspezifischer Systemfunktionen. Zeitschrift für Berufs- und Wirtschaftspädagogik, 115(1), 39-65.

Kärner, T., Warwas, J. \& Schumann, S. (2019). Addressing individual differences in the vocational classroom: towards a Teachers' Diagnostic Support System (TDSS). In T. Deißinger, U. Hauschildt, P. Gonon \& S. Fischer (eds.), Contemporary Apprenticeship Reforms and Reconfigurations, (pp. 179-182). Zürich: Lit.

Kauffmann, H., Knapp, A., Novotny, E. \& Schoch, S. (2002). Flüchtlinge und Schule? Erfahrungen. Zeitschrift für internationale Bildungsforschung und Entwicklungspädagogik, 25(2), 15-19.

Klafki, W. \& Stöcker, H. (2007). Innere Differenzierung des Unterrichts. In W. Klafki (Hrsg.), Neue Studien zur Bildungstheorie und Didaktik. Zeitgemäße Allgemeinbildung und kritisch-konstruktive Didaktik, (S. 173-208). Weinheim: Beltz. 
Kranert, H.-W. \& Stein, R. (im Druck). Multiprofessionalität in der inklusiven Unterrichtsentwicklung - Mögliche Beiträge der Sonderpädagogik in einem interdisziplinären Team an Berufsschulen. In K. Heinrichs \& H. Reinke (Hrsg.), Heterogenität in der beruflichen Bildung im Spannungsfeld zwischen Erziehung, Förderung und Fachausbildung. Reihe Wirtschaft - Beruf - Ethik. Bielefeld: wbv.

Leiprecht, R. \& Lutz, H. (2015). Intersektionalität im Klassenzimmer. Zur sozialen Konstruktion und Bedeutung von Ethnie, Klasse, Geschlecht und ihren Verbindungen. In R. Leiprecht \& A. Steinbach (Hrsg.), Schule in der Migrationsgesellschaft. Ein Handbuch. Band 1: Grundlagen - Diversität-Fachdidaktiken, (S. 283-304). Schwalbach am Taunus: Debus.

Leiprecht, R. \& Steinbach, A. (2015). Einleitung. Schule in der Migrationsgesellschaft. In R. Leiprecht \& A. Steinbach (Hrsg.), Schule in der Migrationsgesellschaft. Ein Handbuch. Band 1: Grundlagen - Diversität-Fachdidaktiken, (S. 7-22). Schwalbach am Taunus: Debus.

Maué, E., Schumann, S. \& Diehl, C. (2018). Bildungshintergrund und Bildungspläne geflüchteter Jugendlicher im System der beruflichen Bildung. In E. Wittmann, D. Frommberger \& B. Ziegler (Hrsg.), Jahrbuch der berufs- und wirtschaftspädagogischen Forschung 2018. Schriftenreihe der Sektion Berufs- und Wirtschaftspädagogik der Deutschen Gesellschaft für Erziehungswissenschaft (DGfE), (S. 137148). Berlin: Budrich.

Paradies, L. \& Linser, H. J. (Hrsg.). (2013). Differenzieren im Unterricht. Berlin: Cornelsen.

Reinke, H., Kärner, T. \& Heinrichs, K. (2018). Analyse lern- und entwicklungsförderlicher Gestaltungsbedingungen beruflicher Praktika für berufsschulpflichtige Asylsuchende und Flüchtlinge: Befunde einer Tagebuchstudie. Unterrichtswissenschaft, 46(1), 43-60.

Roeder, P. M. (1997). Binnendifferenzierung im Urteil von Gesamtschullehrern. Zeitschrift für Pädagogik, 43(2), 241-259.

Scholz, I. (2010). Pädagogische Differenzierung. Stuttgart: UTB.

Schumann, S., Kärner, T. \& Maué, E. (2018). Integration von jungen Geflüchteten in das deutsche Berufsbildungssystem: Klassenkompositionseffekte und binnendifferenzierende Maßnahmen. In M. Pilz, K. Breuing \& S. Schumann (Hrsg.), Berufsbildung zwischen Tradition und Moderne. Festschrift für Thomas Deißinger zum 60. Geburtstag, (S. 83-101). Wiesbaden: Springer VS.

StBA (Statistisches Bundesamt). (2014). Bevölkerung und Erwerbstätigkeit. Bevölkerung mit Migrationshintergrund - Ergebnisse des Mikrozensus. Online: https://www.destatis.de/DE/Publikationen/Thematisch/Bevoelkerung/MigrationIntegration/Migrationshintergrund2010220137004.pdf?_blob=publicationFile (05.05.2016).

Terhart, E. (2015). Umgang mit Heterogenität: Anforderungen an Professionalisierungsprozesse. In C. Fischer (Hrsg.). (Keine) Angst vor Inklusion, (S. 63-83). Münster: Waxmann.

Terrasi-Haufe, E., Hofmann, M. \& Sogl, P. (2018). Sprachförderung in der beruflichen Bildung nach dem Unterrichtskonzept „Berufssprache Deutsch“. Zeitschrift für Interkulturellen Fremdsprachenunterricht, 23(1), 3-16.

Trautmann, M. \& Wischer, B. (2013). Schultheoretische Anfragen zum pädagogischnormativen Heterogenitätsdiskurs. Schweizerische Zeitschrift für Bildungswissenschaften, 35(1), 21-36. 
UNGA (United Nations General Assembly/Generalversammlung der Vereinten Nationen). (1948). Resolution der Generalversammlung 217 A (III). Allgemeine Erklärung der Menschenrechte. Online: http:/www.un.org/depts/german/menschenrechte/aemr.pdf (06.05.2016).

Warwas, J., Kärner, T. \& Golyszny, K. (2015). Diagnostische Sensibilität von Lehrpersonen im Berufsschulunterricht: Explorative Prozessanalysen mittels ContinuousState-Sampling. Zeitschrift für Berufs- und Wirtschaftspädagogik, 111(3), 437454.

Weinert, F. E. (1997). Notwendige Methodenvielfalt. Unterschiedliche Lernfähigkeit erfordert variable Unterrichtsmethoden. In M. A. Meyer, U. Rampillon, G. Otto \& E. Terhart (Hrsg.), Lernmethoden-Lehrmethoden. Wege zur Selbstständigkeit, (S. 50-53). Seelze: Friedrich.

Wischer, B. (2007). Wie sollen LehrerInnen mit Heterogenität umgehen? Über, ,programmatische“ Fallen im aktuelle Reformdiskurs. Die deutsche Schule, 99(4), 422-433.

Wischer, B. \& Trautmann, M. (2012). Innere Differenzierung als reformerischer Hoffnungsträger. Eine einführende Problemskizze zu Leerstellen und ungelösten Fragen. In T. Bohl, M. Bönsch, M. Trautmann \& B. Wischer (Hrsg.), Binnendifferenzierung. Teil 1: Didaktische Grundlagen und Forschungsergebnisse zur Binnendifferenzierung im Unterricht. Theorie und Praxis der Schulpädagogik, (S. 24-39). Immenhausen bei Kassel: Prolog.

Witte, A. \& Harden, T. (2010). Die Rolle des Lehrers/der Lehrerin im Unterricht des Deutschen als Zweit- und Fremdsprache. In H.-J. Krumm, C. Fandrych, B. Hufeisen \& C. Riemer (Hrsg.), Deutsch als Fremd- und Zweitsprache. Ein internationales Handbuch, (S. 1324-1340). Berlin: De Gruyter.

Zoyke, A. (2017). Inklusive Didaktik (wirtschafts-)beruflicher Bildung. Berufsbildung, $71,24-26$. 


\title{
Entwicklung eines fachdidaktischen Kategoriensystems zur Analyse des kognitiv- aktivierenden Potenzials von Aufgaben - ein Beitrag zur Unterrichtsqualitätsforschung in der beruflichen Fachrichtung Pflege
}

\author{
Bärbel Wesselborg, Ulrike Weyland und Marc Kleinknecht
}

\section{Einleitung}

Die kognitive Aktivierung von Lernenden stellt in der Unterrichtsqualitätsforschung, neben den Merkmalen Klassenführung und einem positiven Lernklima, eine von drei Basisdimensionen lernwirksamen Unterrichts dar (Praetorius, Klieme, Herbert \& Pinger 2018; Klieme \& Rakoczy 2008). Eingeführt wurde der Begriff der kognitiven Aktivierung im deutschsprachigen Raum von der Forschergruppe um Baumert und Klieme, um verhaltensnahe Merkmale von Lehrpersonen innerhalb kognitiv-konstruktivistischer Unterrichtsansätze zu beobachten (Baumert et al. 2004; Klieme, Schümer \& Knoll 2001). Verstanden wird darunter ein mehrdimensionales Konstrukt, das ,die Komplexität von Aufgabenstellungen und Argumentationen und die Intensität des fachlichen Lernens" (Klieme et al. 2001, 51) abbildet. Als Kern eines kognitiv-aktivierenden Unterrichts gelten komplexe und problemhaltige Aufgabenstellungen, mit welchen sich Lernende in Arbeitsphasen selbständig auseinandersetzen (Klieme 2018).

Entscheidend für den wissenschaftlichen und unterrichtspraktischen Diskurs zu Anforderungen an kognitiv anspruchsvolle Aufgaben waren die Ergebnisse der TIMSS ${ }^{1}$-Videostudie (Baumert et al. 1997; Klieme et al. 2001). Die Studie zeigte, dass die deutschen Lernenden zwar Routineaufgaben in der Regel lösen konnten, aber Schwierigkeiten hatten, offene und komplexe Aufgaben, die Problemlösefähigkeiten und Verstehensleistungen erforderten, zu bewältigen (Baumert, Klieme \& Bos 2001). Diese Ergebnisse wurden auch mit einem in Deutschland häufig beobachtetem fragend-entwickelnden Unterrichtsstil in Verbindung gebracht, in welchem die Lehrpersonen kleinschrittig Sachverhalte erläuterten und Aufgaben hauptsächlich zum wiederholenden und nachvollziehenden Üben einsetzten (Klieme et al. 2001). Infolgedessen richtete sich der Fokus der empirischen Lehr-Lernforschung in den letzten

1 The Third International Mathematics and Science Study 
zwei Dekaden verstärkt auf die Analyse des Anspruchs- und Aufforderungsgehalts von Lern- und Leistungsaufgaben. Mittlerweile stellt das Thema ,Unterrichtsqualität ' und eine kognitiv-aktivierende Aufgabenkultur in der empirischen Unterrichtsforschung der allgemeinen sowie auch beruflichen Bildung einen Forschungsschwerpunkt dar (u. a. Kunter et al. 2011; Kleinknecht, Maier, Metz \& Bohl 2011b; Minnameier, Hermkes \& Mach 2015; Seifried \& Wuttke 2015). Dabei wird die Frage nach dem Anspruchs- und Aufforderungsgehalt von Aufgaben vornehmlich aus einer fachdidaktischen Perspektive beleuchtet (z. B. Kunter et al. 2011; Wendt et al. 2016). Es liegen vor allem Ergebnisse zu komplexen und problemhaltigen Aufgabenstellungen in den Fächern Mathematik und Naturwissenschaften vor (u. a. Jordan et al. 2006; Jatzwauk 2007).

In der beruflichen Fachrichtung Pflege besteht hingegen ein Mangel an empirisch fundierten Ergebnissen zu Lehr-Lernprozessen, Forschung zu Unterrichtsqualität stellt ohnehin ein Desiderat dar (Darmann-Finck 2015; Nickolaus \& Wuttke 2010). Anders als in den Fachdidaktiken Mathematik und Naturwissenschaften werden Unterrichtsprozesse in der Pflegedidaktik nur punktuell in qualitativen Designs (z. B. Darmann-Finck 2010) oder deskriptiv-statistisch (z. B. Reiber 2010) untersucht. Studien mit inferenzstatistischen Auswertungsverfahren existieren unseres Wissens (im deutschen Kontext) nicht. Zwar hat die Pflegedidaktik in den letzten zwanzig Jahren differenzierte theoretische fachdidaktische Modelle unterschiedlicher Abstraktionsniveaus hervorgebracht (u. a. Darmann-Finck 2010; Ertl-Schmuck 2000), jedoch bedürfen diese der empirischen Absicherung und Ergänzung (Darmann-Finck 2015; Wesselborg 2016).

Aktuell werden in der beruflichen Fachrichtung Pflege in dreijährigen Ausbildungsgängen die Berufe der Alten-, Gesundheits- und (Kinder-)Krankenpflege an Berufsfachschulen oder Schulen, die in der Regel eng mit Einrichtungen des Gesundheitswesens verbunden sind, qualifiziert. Die traditionelle Differenzierung der Ausbildungsgänge nach institutionell getrennten Arbeitsfeldern wird ab dem 1. Januar 2020 durch das generalistisch ausgerichtete Pflegeberufegesetz (PflBG) aufgehoben. Gesetzlich sind die Pflegeausbildungen als Heilberuf durch den Bund über Berufszulassungsgesetze und entsprechende Ausbildungs- und Prüfungsverordnungen und somit außerhalb des Berufsbildungsgesetzes (BBiG) geregelt (Bals \& Dielmann 2013). Die Voraussetzungen zur Tätigkeit als Pflegelehrperson in den Bundesländern sowie den entsprechenden Studiengängen divergieren stark aufgrund unterschiedlicher landesgesetzlicher Regelungen (Wesselborg \& Bögemann-Großheim 2017; Weyland \& Reiber 2013).

Vor dem Hintergrund einer notwendigen Professionalisierung des Berufsbildungspersonals in den Pflegeberufen (Weyland \& Reiber 2017) und der Schlüsselrolle, welche Lehrenden in der Gestaltung von Lehr-Lern-prozessen im Zusammenhang mit Lernerfolg zukommt (Hattie 2010; Baumert \& Kunter 
2011), die auch in pflegequalifizierenden Bildungsgängen angenommen wird, erscheint der Mangel an empirischen Erkenntnissen zu Unterrichtsqualität in der beruflichen Fachrichtung Pflege besonders bedenklich. Dies gilt insbesondere angesichts der aktuellen und zukünftig weiter wachsenden gesellschaftlichen Bedeutung, die den Pflegeberufen bei der demografischen und epidemiologischen Entwicklung und den damit einhergehenden gesundheitlichen Versorgungsbedarfen zugesprochen wird (Scheidt-Nave, Richter, Fuchs \& Kuhlmey 2010).

An dieser spezifischen, auf die berufliche Fachrichtung Pflege zugeschnittenen, Ausgangslage setzt der Artikel an. Er versteht sich als ein perspektivischer Beitrag zur Unterrichtsqualitätsforschung, der seinen Fokus auf die zukünftige Erhebung des kognitiven Potenzials von Aufgaben in der beruflichen Fachrichtung Pflege richtet. Hierzu wird eine fachdidaktische Instrumentenentwicklung skizziert, die sich auf die Anwendung und Weiterentwicklung des allgemeindidaktischen Kategoriensystems zur Erhebung des kognitiv-aktivierenden Aufgabenpotenzials von Kleinknecht et al. (2011b) in der beruflichen Fachrichtung Pflege konzentriert. Ziel der Instrumentenentwicklung ist es, das allgemeindidaktische Kategoriensystem und seine Merkmale pflegedidaktisch zu überprüfen, zu modifizieren und weiterzuentwickeln und anschließend die Reliabilität des erarbeiteten Instruments zu testen. Daneben soll der Beitrag die wissenschaftliche Diskussion zur Aufgabenqualität in der Pflegedidaktik anregen und auf die Relevanz von Mikroprozessen im beruflichen Unterricht hinweisen.

Entsprechend gestaltet sich der Artikel wie folgt: Zunächst wird im Kontext von kognitiv-aktivierenden Aufgaben der Begriff ,Aufgabe' in einem, hier im Artikel fokussierten, engeren (vgl. Kapitel 2.1) und einem weiteren Verständnis sowie hinsichtlich kognitiv-aktivierender Merkmale dargelegt. Darauffolgend werden die Analysekriterien des etablierten, allgemeindidaktischen Kategoriensystems zur Erfassung des kognitiven Potenzials von Aufgaben erläutert (ebd.) und das Vorgehen zur Weiterentwicklung des Kategoriensystems für die berufliche Fachrichtung Pflege beschrieben. Der Beitrag endet mit einem Ausblick auf die weitere Validierung und Anwendung des Instruments in der beruflichen Fachrichtung Pflege.

\section{Kognitiv-aktivierende Aufgaben}

\subsection{Aufgaben im Unterricht}

Im Allgemeinen versteht man unter,Aufgaben“ die Aufforderungen bzw. die Angebote, die zum Denken und Handeln im Unterrichtsprozess anregen (Jor- 
dan et al. 2006). In einer engen Definition werden unter dem Begriff der ,Aufgabe $^{6}$ Aufforderungen und Angebote gefasst, die ein selbständiges Erarbeiten und/oder Üben in Schülerarbeitsphasen intendieren (u. a. Bohl \& Kleinknecht 2009; Jatzwauk 2007; Neubrand 2002). In einer weiten Definition werden unter Aufgaben alle Denk- und Handlungsaufforderungen der Lehrkraft verstanden, also auch Fragen und Impulse im Unterrichtsgespräch (u. a. Kühn 2010; Rumann \& Tiemann 2007). Beide Verständnisse können Gegenstand empirischer Erhebungen sein, wobei in diesem Artikel einem engeren Verständnis von Aufgabe gefolgt wird.

Aufgaben wird eine zentrale Rolle für das Lehren und Lernen in schulischen Kontexten zugeschrieben. Mit ihrer Hilfe können unterschiedliche didaktische Ziele verfolgt werden (Kleinknecht, Bohl, Maier \& Metz 2011a): Lernaufgaben fördern die Lernenden in ihrem Wissens- und Kompetenzerwerb. Sie können dementsprechend in Erarbeitungs-, Übungs- oder Anwendungsaufgaben unterteilt werden. Dabei ist es möglich, in Erarbeitungs-, Übungs- und Anwendungsphasen mehrere Aufgaben oder eine Aufgabe mit impliziten Teilaufgaben (z. B. „beschreibe und erkläre...") einzusetzen. Leistungsaufgaben erheben die Lernstände der Lernenden zu einem bestimmten Zeitpunkt und messen den Lernerfolg (Kleinknecht et al. 2011a; Leisen 2006). Diagnoseaufgaben stellen eine Zwischenform dar, da sie im Sinne eines ,assessment for learning“ (Black, Harrison \& Lee 2003) dazu genutzt werden, Lernprozesse in unmittelbarem Zusammenhang mit der Förderung dieser Prozesse zu erschließen.

Weiterhin können Aufgaben hinsichtlich ihrer Funktion im Unterricht bzw. hinsichtlich des damit verbundenen Lernziels unterschieden werden. Während bspw. für das problemhaltige Erarbeiten von Wissen komplexe Aufgaben eine zentrale Rolle spielen, haben für das Einüben von Routinen auch einfache Übungsaufgaben eine wichtige Bedeutung (Kleinknecht 2019).

Davon abgrenzend wird unter ,Aufgabenkultur ${ }^{6}$ aus einer umfassenden analytisch-deskriptiven Perspektive die Art und Weise verstanden, wie Lehrende und Lernende mit Aufgaben im Unterricht umgehen. Dabei werden neben der Qualität der einzelnen Aufgabe - im Sinne einer Aufforderung bzw. eines Angebotes zum Denken und Handeln - weitere für den Lernerfolg wichtige Aspekte betrachtet, wie z. B. die Lehrperson in die Aufgabenstellung einführt und diese erklärt oder wie die Lernenden bei der Bearbeitung der Aufgabe von der Lehrperson unterstützt werden (Bohl \& Kleinknecht 2009).

\subsection{Kognitiv-aktivierende Aufgaben}

In kognitionspsychologischen und konstruktivistischen Theorien des Lernens, die in der Tradition von Piaget stehen, wird davon ausgegangen, dass anspruchsvolle Aufgaben und eine diskursive Unterrichtsgestaltung kognitive 
Konflikte auslösen, die problemlösende sowie metakognitive Denkprozesse der Lernenden initiieren, die ein vertieftes Verständnis für fachliche Inhalte fördern (Klieme 2018).

Als zentrale Auslöser für die kognitive Aktivierung gelten die Aufgaben und Fragen, die die Lehrkraft während des Unterrichts den Lernenden stellt und die ,zum vertieften Nachdenken und zu einer elaborierten Auseinandersetzung mit dem Unterrichtsgegenstand“ (Lipowsky 2015, 89) anregen. Entsprechend zielt das Konstrukt der kognitiven Aktivierung nicht auf eine äußerlich zu beobachtende Aktivität der Lernenden (,,behavioral activity“), sondern auf das Angebot an Anregungen und Aufgaben, das Lernenden anspruchsvolles bzw. vertieftes Denken (,,cognitive activity“) ermöglicht (Mayer 2004, 17).

Demzufolge zeichnen sich hochkognitiv-aktivierende Aufgaben dadurch aus, dass sie bei der Bearbeitung kognitive Konflikte bei den Lernenden hervorrufen und unterschiedliche Ideen, Positionen, Interpretationen und Lösungen ermöglichen. Dabei werden die Lernenden angeregt, ihre Gedanken, Konzepte und Lösungswege in die Aufgabenbearbeitung einzubringen, zu begründen, die Tragfähigkeit ihrer Überlegungen einzuschätzen und mit anderen möglichen Lösungsansätzen zu vergleichen. Entsprechend findet eine kognitive Aktivierung der Lernenden statt, wenn es zu fachlich anspruchsvollen Auseinandersetzungen in der Klasse kommt und/oder neue Unterrichtsinhalte mit dem Vorwissen der Lernenden verknüpft werden können. Demgegenüber sind eng gestellte Fragen und Aufgaben, die nur einfache Antworten ohne Begründung erfordern, für die kognitive Aktivität der Lernenden und nachhaltigen Wissenskonstruktionsprozesse unzureichend (Lipowsky 2015).

In der Unterrichtsforschung wird davon ausgegangen, dass das Unterrichtsqualitätsmerkmal der kognitiven Aktivierung über Analysen von Aufgaben und des Lehrerverhaltens in Unterrichtsvideografien eingeschätzt werden kann. Dagegen ist die kognitive Aktivität der Lernenden nicht direkt messbar. Sie muss approximativ in Videoanalysen oder mittels Schülerfragebögen über die wahrgenommenen Elaborationsprozesse der Lernenden erhoben werden (Lipowsky 2015). Da nicht direkt von Aufgabenmerkmalen auf die kognitiven Prozesse der Lernenden geschlossen werden kann, wird häufig vom Potenzial der kognitiven Aktivierung in Aufgabenstellungen gesprochen (Neubrand, Jordan, Krauss, Blum \& Löwen 2011). Empirisch erweist sich das Potenzial der kognitiven Aktivierung, erfasst über Klassenarbeitsaufgaben und die von den Lernenden eingeschätzte kognitive Aktivierung im Mathematikunterricht, als signifikanter Prädiktor für Lernzuwachs (Baumert \& Kunter 2011).

Trotz der vorgestellten konsensfähigen Charakterisierung des Begriffs ,kognitive Aktivierung' wird dieser in der empirischen Unterrichtsforschung, bedingt durch unterschiedliche Forschungsdesigns und den verbundenen Erhebungsmethoden und -perspektiven, unterschiedlich definiert und operationalisiert (für einen Überblick: Hanisch 2018; Praetorius et al. 2018). 


\section{Allgemeindidaktisches Kategoriensystem zur Erfassung des kognitiv-aktivierenden Potenzials von Aufgaben}

Ausgehend von der Entwicklung fachdidaktischer Kategoriensysteme zur Aufgabenanalyse, bspw. der Mathematik- und Biologiedidaktik (u. a. Jordan et al. 2006; Jatzwauk 2007), wurden in den letzten Jahren auch in der Allgemeinen Didaktik differenzierte Klassifikationssysteme zur Analyse des kognitiv-aktivierenden Potenzials von Aufgaben (Kleinknecht et al. 2011b) bzw. von einer kognitiv-aktivierenden Aufgabenkultur (Blömeke, Risse, Müller, Eichler \& Schulz 2006) erarbeitet.

Für den Bereich der beruflichen Bildung in der Fachrichtung Pflege wird in dieser Arbeit vorgeschlagen, das Kategoriensystem von Kleinknecht und Kollegen als Basisinstrument zur Erhebung des kognitiven Potenzials von Aufgaben heranzuziehen (2011b). Zu beachten ist, dass das Kategoriensystem kein umfassendes didaktisches Instrument zur Planung und Analyse von Aufgaben darstellt (hierzu Blömeke et al. 2006). Es fokussiert auf objektive Qualitätsmerkmale kognitiv-aktivierender Aufgaben aus Expertensicht und berücksichtigt nicht die unterrichtliche Einbettung und Sequenzierung (Kleinknecht et al. 2011b). Das Instrument eignet sich im Besonderen, da es allgemeindidaktisch und dementsprechend fachübergreifend angelegt ist und den Anspruch verfolgt, Aufgabenanforderungen in unterschiedlichen didaktischen Settings, also Lern- sowie Leistungsaufgaben, zu erfassen (ebd.). Das Instrumment wurde von Experten bereits in unterschiedlichen Domänen und auch zu fachdidaktischen Analysen von Aufgaben im Wirtschaftsunterricht eingesetzt (Arndt 2013).

Das Instrument wurde mittels eines quantitativ inhaltsanalytischen Vorgehens entwickelt (Kleinknecht et al. 2011b). Ausgangspunkt stellten fachdidaktische Kategoriensysteme zur Aufgabenanalyse dar (u. a. Jordan et al. 2006). In der Entwicklung wurden die Analysekriterien der fachdidaktischen Instrumente bezüglich überfachlicher Eignung geprüft und entsprechend adaptiert und verfeinert (Kleinknecht et al. 2011b). Anschließend wurde das Instrument erprobt und die Reliabilität mittels 127 Lern- und Leistungsaufgaben aus verschiedenen Fächern überprüft. Dabei zeigten sich gute bis sehr gute Übereinstimmungswerte (ebd.).

Im Zentrum steht die Analyse des kognitiv-aktivierenden Potenzials von Aufgaben mit den Dimensionen ,Wissensart' und ,kognitiver Prozess' (v.a. Anderson \& Krathwohl 2001). Dabei bezieht sich das Instrument auf die von Anderson und Krathwohl entwickelte revidierte Bloom'sche Taxonomie. Anders als die von Bloom und Kollegen eindimensional angelegten und in sechs Stufen geordneten kognitiven Prozesskategorien werden in dem mehrdimensional aufgebauten Kategoriensystem sowohl die kognitiven Prozesse als auch 
die Wissensart analysiert. Dabei umfasst die Dimension, Wissensart' die Kategorien ,Faktenwissen', ,prozedurales Wissen', ,konzeptuelles Wissen“ und ,metakognitives Wissen" und orientiert sich an kognitionspsychologischen Konzepten (Kleinknecht et al. 2011b, 334). Bspw. wird eine Aufgabe unter Faktenwissen kodiert, wenn in der Aufgabe verbalisierbares und für eine bestimmte Fachdomäne relevantes Wissen oder isolierte komplexe Fakten ohne Anwendungswissen erfragt wird, wie die Nennung bestimmter Fachbegriffe bei der Beschriftung einer Abbildung. Die Dimension ,kognitiver Prozess“ wird von der ursprünglichen Bloom'schen Taxonomie übernommen, jedoch entscheiden sich die Autoren zu einer reduzierten vierstufigen Konzeptualisierung mit dem Fokus auf die oberen Taxonomiestufen. Sie diversifizieren in die Kategorien ,Reproduktion', ,naher Transfer', ,weiter Transfer' und ,Problemlösen` (a. a. O., 335), ähnlich anderer fachdidaktischer Ansätze, die nur zwischen den drei Taxonomiestufen Wissen, Verstehen und Anwenden unterscheiden (z. B. Schabram 2007). Dabei wird z. B. kreative Problemlöseaufgabe kodiert, wenn in der Aufgabenstellung eine komplexe Fallsituation geschildert wird und zunächst unklar ist, welches Wissen in welcher Kombination zur Anwendung kommen soll. Die Abbildung 1 zeigt das gesamte Kategoriensystem:

Abb. 1: Kategoriensystem zur Erfassung des kognitiven Potenzials von Aufgaben

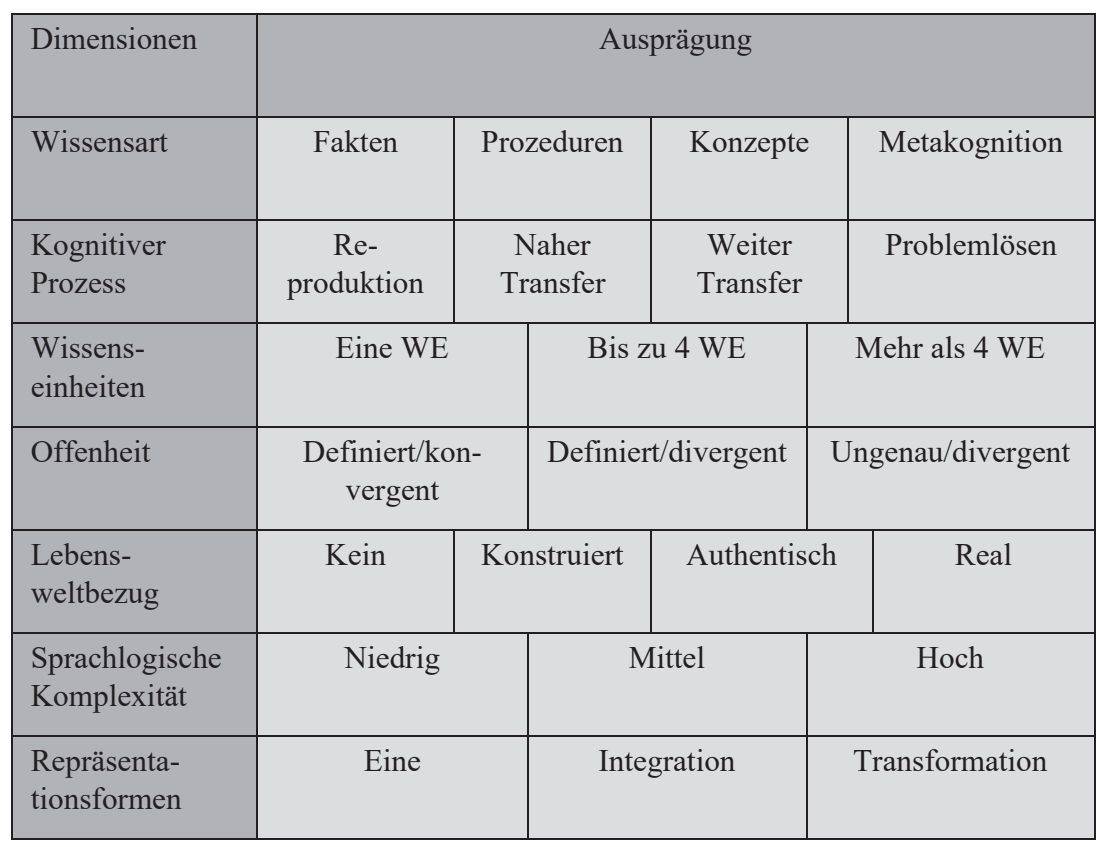

Quelle: Kleinknecht et al. 2011b, 334 
Neben den zwei Dimensionen ,Wissensart' und ,kognitiver Prozess ‘ wurden weitere fünf Kategorien aus fachdidaktischen Kategoriensystemen adaptiert sowie Dimensionen aus der allgemeindidaktischen Diskussion zur Aufgabengestaltung genutzt (Kleinknecht et al. 2011b).

Die Dimension ,Wissenseinheiten ' bezieht sich auf die Komplexität von Aufgaben. In dieser Dimension werden die zu aktivierenden fachspezifischen Wissenselemente aus der Sicht von Fachexperten analysiert (ebd.). Wenn ein Fakt oder Konzept erfragt wird, bleibt es bei einer Wissenseinheit. Aufgaben mit mehr als vier Wissenseinheiten sind wenig anspruchsvoll, wenn sie sich auf der Ebene des Faktenwissens oder des prozeduralen Wissens bewegen, z. B. nach vier Konzepten gefragt wird. Komplexer ist, wenn verschiedene Prozeduren und Konzepte kombiniert werden müssen. Dies ist z. B. bei Aufgaben der Fall, in welchen Texte produziert werden müssen, die eine Kombinationsleistung zwischen domänenspezifischem Wissen und grammatikalischem bzw. Rechtschreibwissen erfordern.

Bei dem Analysekriterium ,Offenheit ' wird eingeschätzt, inwieweit bei der zu lösenden Aufgabe, bezogen auf den Anfangs- und Zielzustand sowie den Transformationsprozess, jeweils Geschlossenheit bzw. Offenheit vorliegt. Dabei wird zum einen berücksichtigt, ob die zu lösende Aufgabe deutlich beschrieben ist oder nicht. Zum anderen wird berücksichtigt, ob konvergente Aufgaben, die eine Lösung erfordern und divergente Aufgaben, die mehrere Lösungen erlauben, vorliegen (ebd.).

Die Dimension ,Lebensweltbezug ' bezieht die didaktische und lerntheoretische Forderung ein, schulische Inhaltsangebote auf die Alltags- und Lebenswelt der Schülerinnen und Schüler auszurichten, damit es zu vertieften Elaborationsprozessen kommt (Renkl 2015). Aufgaben können auf einen Lebensweltbezug verzichten oder aber einen konstruierten bzw. sogar einen authentischen Lebensweltbezug berücksichtigen, der Lerndende auffordert, sich mit tatsächlich zu lösenden Problemstellungen aus ihrer Lebenswelt auseinanderzusetzen (Kleinknecht et al. 2011b).

Die Dimension ,sprachlogische Komplexität analysiert die sprachliche Komplexität der Aufgaben und inwiefern Aufgabentexte und Bearbeitungsschritte strukturgleich sind (ebd.).

Das Analysekriterium ,Repräsentationsformen“ zielt ebenfalls auf die Komplexität von Aufgaben und berücksichtigt, inwieweit die Lernenden eine Umgestaltung der Darstellung der Inhalte durchführen sollen, die so in der Aufgabenstellung nicht vorkommen wie z. B. die Transfomation von Texten in Grafiken (Kleinknecht et al. 2011b). 


\section{Entwicklung eines Instruments zur Erhebung des kognitiv-aktivierenden Potenzials von Aufgaben in der beruflichen Fachrichtung Pflege}

Durch die fachübergreifende empirische Entwicklung des Instruments kann angenommen werden, dass das allgemeindidaktische Kategoriensystems zur Erhebung des kognitiv-aktivierenden Potenzials von Aufgaben von Kleinknecht et al. (2011b) ebenfalls in der beruflichen Fachrichtung Pflege zu einem Erkenntnisgewinn hinsichtlich der Aufgabenqualität führt. Aufgaben, die der Pflegeunterricht anbietet, können auf diese Weise mit einem überfachlichen Ansatz bezogen auf ihr kognitives Aktivierungspotenzial analysiert werden. Allerdings bleibt dabei unbeantwortet, ob das Gesamtkonstrukt mit seinen Dimensionen und Ausprägungskennzeichnungen in ausreichendem Maße und widerspruchsfrei die fachspezifischen Anforderungen des Pflegeunterrichts erfassen kann. Deshalb wäre eine fachdidaktische Überprüfung und ggf. Weiterentwicklung wichtig, um den spezifischen Bedarfen und Bedingungen von Pflegeunterricht Rechnung tragen zu können.

Auch empirische Befunde verweisen auf die Notwendigkeit fachdidaktischer Absicherung des Konstrukts der kognitiven Aktivierung. So gibt es Hinweise aus der Unterrichtsforschung, dass im Vergleich zu den Unterrichtsqualitätsmerkmalen ,effektive Klassenführung' und ,Unterrichtsklima' die Dimension ,kognitive Aktivierung' weniger stabil ist. Es zeigte sich in Videoanalysen, dass die Ausprägung der Basisdimension ,kognitive Aktivierung ‘ von Unterrichtseinheit zu Unterrichtseinheit variieren kann und offenbar stärker von Merkmalen des zu unterrichtenden Inhalts abhängt (Praetorius et al. 2014). Daher kann geschlussfolgert werden, dass die Basisdimension ,kognitive Aktivierung' inhaltsabhängig ist und in Abhängigkeit zur fachdidaktischen Kompetenz der Lehrenden steht. Dementsprechend wird bereits seit längerem gefordert, dass mittels fachdidaktischer Forschung konkretisiert werden solle, was kognitive Aktivierung in dem jeweiligen Fach bedeute (Klieme \& Rakoczy 2008).

Diesem Anspruch wird im Folgenden nachgegangen und die Anwendung und Weiterentwicklung des allgemeindidaktischen Kategoriensystems zur Erhebung des kognitiv-aktivierenden Aufgabenpotenzials von Kleinknecht et al. (2011b) in der beruflichen Fachrichtung Pflege fokussiert. Wie eingangs beschrieben (siehe Kapitel 1) wird bei der Instrumentenentwicklung das Ziel verfolgt, die allgemeindidaktischen Analysekriterien pflegedidaktisch zu überprüfen, zu modifizieren und weiterzuentwickeln und anschließend die Messqualität des erarbeiteten Instrumentes empirisch zu evaluieren. Forschungsmethodisch orientiert sich die Instrumentenentwicklung an der Inhaltsanalyse nach 
Bos und Tarnai (1999). Diese ermöglicht ein systematisches und gütekriteriengeleitetes Vorgehen, wobei die Kategorienbildung bzw. -anpassung deduktiv als auch/oder induktiv erfolgen kann.

Die folgende Abbildung 2 zeigt die Schritte des Entwicklungsprozesses des pflegedidaktischen Instruments auf:

Abb. 2: Entwicklung und Überprüfung eines pflegedidaktischen Kategoriensystems zur Analyse des kognitiv-aktivierenden Potenzials von Aufgaben

Basisinstrument: Allgemeindidaktisches Kategoriensystem zur Analyse des kognitiven Potenzials von Aufgaben

$\downarrow$

Theoriegeleitete pflegedidaktische Überprüfung und Validierung der Kategorien, ggf. Modifizierung und Ergänzung (u. a. Kategorie „Lebensweltbezug" in „Berufs- und Lebensweltbezug"

Theoriegeleitete Überprüfung und Validierung der Kategorien anhand Pflegedidaktikmodellen (u. a. Interaktionistische Pflegedidaktik) und ggf. Modifizierung und Ergänzung

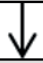

Erster Materialdurchlauf: Kodierung von Pflegeaufgaben, ggf. induktive Kategorienbildung bzw. -modifizierung (u. a. „Fallarbeit", „Berücksichtigung der doppelten Handlungslogik“) Reliabilitätstest, Vergleich der Ergebnisse und Diskussion im Team

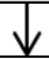

Gesamter Materialdurchlauf: Kodierung von Pflegeaufgaben Reliabilitätstest

Quelle: Eigenentwicklung orientiert an Bos \& Tarnai 1999

An der Instrumentenentwicklung sind vier Expertinnen, zwei Pflegedidaktikerinnen und zwei Fachlehrerinnen mit einem berufspädagogischen Studium der Fachrichtung Pflege, beteiligt. Voraussetzung war eine vertiefte Kenntnis zu Pflegedidaktik und pflegedidaktischen Modellen, Aufgabenkonstruktion und Erkenntnissen der Unterrichtsqualitätsforschung insbesondere hinsichtlich des Merkmals der kognitiven Aktivierung. 
Als Untersuchungsmaterial dienen Aufgaben der schulischen (Kinder-) Kranken- und Altenpflegeausbildung, die von kooperierenden Pflegeschulen zur Verfügung gestellt wurden. Die Aufgaben repräsentieren einen Querschnitt an unterschiedlichen didaktischen Funktionen aus allen drei Ausbildungsjahren der Pflegeberufe: Leistungsaufgaben in Form von Klassenarbeiten oder Abschlussarbeiten sowie Lernaufgaben aus dem Unterricht. Dabei wurden die Schulen aufgefordert, Lernaufgaben einzureichen, die problemlösende Anteile haben, da erwartet wird, dass diese für die induktive Kategorienbildung einen höheren Mehrwert haben. Weiterhin werden Aufgaben aus innovativen pflegedidaktischen Veröffentlichungen und Lehrbüchern einbezogen.

In dem Entwicklungsprozess des Instruments wurden bereits die bekannten allgemeindidaktischen Kategorien in Bezug auf pflegedidaktische Theorien überprüft und eine theoriegeleitete Modifikation bzw. Ergänzung vorgenommen. So untersucht das Kategoriensystem von Kleinknecht et al. (2011b) in der Dimension ,Lebensweltbezug', ob Aufgaben den Lebens- und Erfahrungsraum der Lernenden einbeziehen. In dem pflegedidaktisch modifizierten Instrument wurde die Dimension ,Lebensweltbezug' in ,Berufs- und Lebensweltbezug' verändert, da die Inhalte, die der Pflegeunterricht anbietet primär auf die berufliche Arbeits- und Anforderungssituation der Lernenden gerichtet sind (Krankenpflegegesetz (KrPflG) §3; Darmann-Finck 2010). Durch den hier gesetzten Bezug auf Berufs- und Lebenswelt soll auf die doppelte Zielperspektive beruflicher Bildung hingewiesen werden, welche hier präferiert wird.

Im nächsten, derzeit laufenden, aber noch nicht abgeschlossenen Prozessschritt wird geprüft, inwieweit die bestehenden Analysekriterien durch die Expertinnen theoriegeleitet, ausgehend von den Annahmen von Pflegedidaktikmodellen, inhaltlich valide modifiziert werden können. Hierbei ist es möglich die Kategorien anhand Pflegedidaktikmodellen zu präzisieren bzw. deduktiv ergänzende Kategorien zu entwickeln. Am Beispiel der empirisch begründeten interaktionistischen Pflegedidaktik von Darmann Finck (2010) soll dies exemplarisch veranschaulicht werden: Darmann-Finck hat im Rahmen von Unterrichtsbeobachtungen drei grundsätzliche Bildungskonzepte identifiziert, die Pflegelehrpersonen in ihrer Unterrichtsarbeit einsetzen: Sie unterscheidet zwischen regel-, fall- und meinungsorientierten Konzepten. In einer Heuristik formuliert Darmann-Fink anhand der jeweiligen Konzepte normative Zieldimensionen für die angemessene Thematisierung und Reflexion der Bildungskonzepte im Unterricht (ebd.). Über die normativen Zieldimensionen werden pflegedidaktisch entwickelte Kategorien erkennbar, die die Modifikation, Ergänzung bzw. besondere Akzentuierung von Merkmalen des allgemeindidaktischen Kategoriensystems anregen können. Die Unterrichtskonzepte, die Darmann-Finck mittels Unterrichtsbeobachtungen ermittelt hat, lassen sich im Kategoriensystem von Kleinknecht et al. (2011b) der Dimension, Wissensart ${ }^{`} \mathrm{zu}-$ ordnen. Bei einer pflegedidaktischen Erweiterung könnte das Bildungskonzept 
der Regelorientierung folgendermaßen differenziert werden: Unter dem Bildungskonzept ,Regelorientierung' werden Kommunikationsmuster im Unterricht zusammengefasst, die allgemeine Handlungsregeln für typische Pflegesituationen thematisieren (Darmann-Finck 2010). Die interaktionistische Pflegedidaktik fordert, dass die Handlungsregeln in dieser Zieldimension, wenn möglich, evidenzbasiert bzw. wissenschaftlich fundiert begründet werden sollten (ebd.). Hier wäre es möglich, die allgemeindidaktische Dimension ,Wissensart ${ }^{6}$ pflegedidaktisch zu spezifizieren und in ,praxisbezogene tradierte Handlungsanweisungen', , argumentativ begründete Handlungsstrategien ' und in ,Handlungsstrategien, die auf prinzipiell überprüfbaren wissenschaftlich fundierten Erkenntnissen fußen', weiter zu untergliedern, um das Ausmaß an Aufklärung, das sich Lernende über ihr berufliches Handlungsfeld aneignen können, differenziert zu fassen ${ }^{3}$.

Nach der theoriegeleiteten pflegdidaktischen Modifizierung und deduktiven Entwicklung von neuen Kategorien ist im nächsten Prozessschritt geplant, die inhaltlichen Beschreibungen der Kategorien entsprechend anzupassen bzw. $\mathrm{zu}$ operationalisieren und passende Indikatoren zu formulieren. Anschließend soll ein erster Validitäts- und Reliabilitätstest des Instruments folgen, in welchem die vier Expertinnen unabhängig voneinander mehrere Lern- und/oder Leistungsaufgaben aus Pflegeunterrichten hinsichtlich des kognitiv-aktivierenden Aufgabenpotenzials einschätzen. Die Ergebnisse dieser Untersuchung werden im Anschluss bezogen auf Reliabilität mittels der Erhebung von Reliabilitätskoeffizienten überprüft (Wirtz \& Caspar 2002). Je nach Ergebnis des Übereinstimmungswerts ist vorgesehen, die Einschätzungen von den Expertinnen zu diskutieren und die Validität der Kategorien zu vergleichen. Anschließend werden die bestehenden Kategorien ggf. überarbeitet und verfeinert.

Im Prozess der Instrumentenentwicklung können ebenfalls induktiv Kategorien entwickelt werden. Dabei kann mit Hilfe des Untersuchungsmaterials und der darin enthaltenen impliziten und expliziten Anforderungen nichtkodierte Aspekte, die aus pflegedidaktischer Sicht von Bedeutung sind, diskutiert, entwickelt, operationalisiert und in das bestehende Kategoriensystem aufgenommen werden. Dies wird im Folgenden anhand von Aufgaben, die in Fallgeschichten eingebunden sind, erläutert. Fallgeschichten werden in der Pflegebildung als Medium für Lernprozesse bevorzugt eingesetzt (u. a. Dietrich \& Reiber 2014; Darmann-Finck, Böhnke \& Straß 2009). Fachdidaktisch wird die Präferenz von Fallarbeit mit der Besonderheit von pflegeberuflichen Anforderungssituationen begründet. Um qualitativ hochwertige Pflege zu sichern,

3 Zu bedenken ist, dass ebenso bei Pflegeunterrichtsaufgaben, die evidenzbasierte Erkenntnisse einbeziehen, eine kritische Auseinandersetzung der Lernenden im Umgang mit wissenschaftlichen Ergebnissen zu fördern ist, in dem das Design der Studie hinsichtlich der Fragestellung geprüft und bewertet wird. 
müssen Lernende befähigt werden, nicht nur wissenschaftlich fundiertem Regelwissen Rechnung zu tragen, sondern sie müssen gleichwertig auch das Erleben, die Bedürfnisse, Verständnisse und Erwartungen der pflegebedürftigen Person in ihre Entscheidungen und in ihr Handeln mit einbeziehen. Patienten werden in der humanen Dienstleistung ,Pflege' nicht nur als Objekt des Handelns, sondern als Subjekte und Co-Produzenten pflegerischer Aufgabenbewältigung wahrgenommen. Das Handeln ist somit der doppelten Logik sozialen Handelns unterworfen (vgl. Dewe, Ferchhoff \& Radke 1992; Remmers 2000). Fallarbeit wird eingesetzt, um Lernangebote zu ermöglichen, ,,durch die eine situationsbezogene und relationale Bezugnahme auf diverse Wissensformen sowie reflexiv-hermeneutische Verständigung eingeübt wird" (Walter 2015, 195). Fälle sollen authentisch sein und besonderen theoretischen Anforderungen entsprechen, um zur Auseinandersetzung mit widersprüchlichen Anforderungen aufzufordern (vgl. Darmann-Finck et. al. 2009). Bei der induktiven Kategorienbildung in Bezug auf Aufgaben mit Fallgeschichten könnten entsprechend Kodes entwickelt werden, die die Besonderheit der Anforderungssituation in der Pflege abbilden. Dabei könnten die Kodes bspw. beschreiben, inwieweit die Fallgeschichte die berufliche Anforderungssituation realistisch und ausreichend komplex spiegelt oder die Fallgeschichte der doppelten Handlungslogik entspricht.

Nach der Überarbeitung soll im letzten Prozessschritt die gesamte Aufgabenstichprobe $(n=130)$ mittels des pflegedidaktisch modifizierten bzw. ergänzten Instrumentes von den Expertinnen unabhängig voneinander analysiert werden. Die Ergebnisse werden abschließend einer Reliabilitätsprüfung zur Erhebung der Güte unterzogen. Anschließend werden die Ergebnisse interpretiert und diskutiert (Bos \& Tarnai 1999).

\section{Ausblick}

Im Zusammenhang mit Unterrichtsentwicklung an Pflegeschulen kann das pflegedidaktische Kategoriensystem Pflegelehrenden valide und reliable Orientierungspunkte für die begründete Konstruktion von kognitiv-aktivierenden Lern- und Leistungsaufgaben im Pflegeunterricht und deren Reflexion bieten. Gleichwohl ist bei dem Einsatz des Instruments im Pflegeunterricht notwendig, dass die Pflegelehrpersonen die Lernvoraussetzungen der Auszubildenden und die didaktischen Ziele des Unterrichts einbeziehen, um das Anforderungsniveau der Aufgaben, verbunden mit dem entfalteten Potenzial der kognitiven Aktivierung, angemessen einschätzen zu können. Dabei ist es sinnvoll zuerst anspruchsvolle Lernangebote zu planen und erst im nächsten Schritt mögliche Differenzierungen für ein adaptives Vorgehen im Unterricht vorzunehmen, da 
empirische Befunde zeigen, dass Aufgaben im deutschen (mathematisch-naturwissenschaftlichen) Unterricht, und es wird angenommen, dass dies auch auf Pflegeunterricht zutrifft, überwiegend auf wenig komplexes Denken zielen (z. B. Jatzwauk 2007; Schabram 2007, siehe auch Kleinknecht 2019). Nicht erfasst werden kann mit dem Instrument, wie die Lehrperson die objektiv festgestellten Qualitätsmerkmale der eingesetzten Aufgabe im Unterricht umsetzt oder ausbaut.

Perspektivisch dient das pflegedidaktische Instrument als valide Grundlage zur Erhebung des kognitiven Potenzials von Aufgaben im Rahmen des Paradigmas der Unterrichtsqualitätsforschung in der beruflichen Fachrichtung Pflege. Zur Überprüfung der Konstrukt- und Kriteriumsvalidität des Instruments ist eine mehrperspektivische Studie notwendig, die die eingeschätzte kognitive Aktivierung durch die Lehrperson (Beobachter- und Lehrendenperspektive) und die eingeschätzte kognitive Aktivität der Lernenden (Schülerperspektive) (Clausen 2002) sowie eine Aufgabenanalyse im Zusammenhang mit der Lernleistung im Pflegeunterricht untersucht (Moosbrugger \& Kelava 2012).

Eine mehrperspektivische Studie unter Hinzuziehung der Beobachterperpektive hätte den hohen Mehrwert, die Implementation und den Umgang der Lehperson mit der Aufgabe im Unterricht einschätzen zu können. Dies ist besonders relevant, da in der TIMS-Studie gezeigt werden konnte, dass die Ausgangskomplexität der eingesetzten Mathematikaufgaben nicht unter dem anderer Länder lag, es aber durch das schrittweise Zerteilen der Aufgabe, im stark von der Lehrperson gelenkten fragend-entwickelndem Unterrichtsgespräch, zu einer deutlichen Reduktion der Komplexität und damit zu einer geringeren kognitiven Aktivierung kam (Klieme et al. 2001).

\section{Literatur}

Anderson, L. W. \& Krathwohl, D. R. (Hrsg.). (2001). A Taxonomy for Learning, Teaching and Assessing: A Revision of Bloom's Taxonomy of Educational Objectives. New York: Longman.

Arndt, H. (2013). Fachdidaktische Analysen von Aufgaben im Wirtschaftsunterricht. In M. Kleinknecht, T. Bohl, U. Maier \& K. Metz (Hrsg.). Lern- und Leistungsaufgaben im Unterricht. Fächerübergreifende Kriterien zur Auswahl und Analyse, (S. 193-206). Bad Heilbrunn: Klinkhardt.

Bals, T. \& Dielmann G. (2013). Neugestaltung der Gesundheitsberufe im Kontext des Deutschen Berufsbildungssystems. In Robert Bosch Stiftung (Hrsg.). Gesundheitsberufe neu denken Gesundheitsberufe neu regeln. Grundsätze und Perspektiveneine Denkschrift der Robert Bosch Stiftung, (S. 177-191). Stuttgart: RBS. 
Baumert, J., Klieme, E. \& Bos, W. (2001). Mathematische und naturwissenschaftliche Bildung am Ende der Schullaufbahn - Die Herausforderung von TIMMSS für die Weiterentwicklung des mathematischen und naturwissenschaftlichen Unterrichts. In Bundesministerium für Bildung und Forschung (Hrsg.), TIMSS - Impulse für Schule und Unterricht. Forschungsbefunde, Reforminitiativen, Praxisberichte und Video-Dokumente, (S. 11-42). Bonn: BMBF.

Baumert, J. \& Kunter, M. (2011). Das mathematikspezifische Wissen von Lehrkräften, kognitive Aktivierung im Unterricht und Lernfortschritte von Schülerinnen und Schülern. In M. Kunter, J. Baumert, W. Blum, U. Klusmann, S. Krauss, \& M. Neubrand (Hrsg.), Professionelle Kompetenz von Lehrkräften. Ergebnisse des Forschungsprogramms COACTIV, (S. 163-192). Münster: Waxmann.

Baumert, J., Kunter, M., Brunner, M., Krauss, S., Blum, W. \& Neubrand, M. (2004). Mathematikunterricht aus der Sicht der PISA-Schülerinnen und -Schüler und ihrer Lehrkräfte. In M. Prenzel, J. Baumert, W. Blum, R. Lehmann, D. Leutner, M. Neubrand, R. Pekrun, H.-G. Rolff, J. Rost, \& U. Schiefele (Hrsg.), PISA 2003: Der Bildungsstand der Jugendlichen in Deutschland. Ergebnisse des zweiten internationalen Vergleichs, (S. 314-354). Münster: Waxmann.

Baumert, J., Lehmann, R., Lehrke, M., et al. (Hrsg.). (1997). TIMSS - Mathematischnaturwissenschaftlicher Unterricht im internationalen Vergleich. Deskriptive Befunde. Opladen: Leske + Budrich.

Black, P., Harrison, C. \& Lee, C. (eds.). (2003). Assessment for Learning: Putting it into practice. Maidenhead, U.K.: Open university Press.

Blömeke, S., Risse, J., Müller, C., Eichler, D. \& Schulz, W. (2006). Analyse der Qualität von Aufgaben aus didaktischer und fachlicher Sicht. Ein allgemeines Modell und seine exemplarische Umsetzung im Unterrichtsfach Mathematik. Unterrichtswissenschaft, 34(4), 330-357.

Bohl, T. \& Kleinknecht, M. (2009). Aufgabenkultur. In S. Blömeke, T. Bohl, L. Haag, G. Lang-Wojtasik \& W. Sacher (Hrsg.), Handbuch Schule. Theorie-Organisation -Entwicklung, (S. 331-334). Bad Heilbrunn: Klinkhardt.

Bos, W. \& Tarnai, C. (1999). Content analysis in empirical social research. International Journal of Educational Research, 31(8), 659-671.

Clausen, M. (2002). Unterrichtsqualität: eine Frage der Perspektive? Empirische Analysen zur Übereinstimmung, Konstrukt- und Kriteriumsvalidität. Münster: Waxmann.

Darmann-Finck, I. (Hrsg.). (2010). Interaktion im Pflegeunterricht. Peter Lang: Frankfurt am Main.

Darmann-Finck, I. (2015). Berufsbildungsforschung in den Gesundheitsfachberufen auf dem Weg zu einer Agenda. bwp@ Spezial 10, 1-15. Online: http://www.bwpat.de/spezial10/darmann-finck_gesundheitsbereich-2015.pdf (01.10.2018).

Darmann-Finck, I., Böhnke, U. \& Straß, K. (Hrsg.). (2009). Fallrekonstruktives Lernen. Frankfurt am Main: Mabuse.

Dewe, B., Ferchhoff, W. \& Radtke, F. O. (Hrsg.). (1992): Erziehen als Profession. Zur Logik professionellen Handelns in pädagogischen Feldern. Opladen: Leske + Budrich.

Dietrich, J. \& Reiber K. (Hrsg.). (2014). Fallbasierte Unterrichtsgestaltung. Grundlagen und Konzepte. Stuttgart: Kohlhammer. 
Ertl-Schmuck, R. (2010). Subjektorientierte Pflegedidaktik. In R. Ertl-Schmuck \& F. Fichtmüller (Hrsg.), Theorien und Modelle der Pflegedidaktik. Eine Einführung, (S. 55-90). Weinheim, München: Juventa.

Hanisch, A.-K. (Hrsg.). (2018). Kognitive Aktivierung im Rechtschreibunterricht. Eine Interventionsstudie in der Grundschule. Münster: Waxmann.

Hattie, J. (ed.). (2009). Visible learning: a synthesis of over 800 meta-analyses relating to achievement. New York: Routledge.

Jatzwauk, P. (Hrsg.). (2007). Aufgaben im Biologieunterricht - eine Analyse der Merkmale und des didaktisch-methodischen Einsatzes von Aufgaben im Biologieunterricht. Berlin: Logos.

Jordan, A., Baumert, J., Blum, W., Brunner, M., Kraus, S., Kunter, M., Löwen, K., Neubrand, M. \& Ross, N. (Hrsg.). (2006). Klassifikationsschema für Mathematikaufgaben: Dokumentation der Aufgabenkategorisierung im Coactiv-Projekt. Berlin: Max-Planck-Institut für Bildungsforschung.

Kleinknecht, M. (2019). Aufgaben und Aufgabenkultur. Zeitschrift für Grundschulforschung, 2(1), 1-14.

Kleinknecht, M., Bohl, T., Maier, U. \& Metz, K. (Hrsg.). (2013). Lern- und Leistungsaufgaben im Unterricht. Fächerübergreifende Kriterien zur Auswahl und Analyse. Bad Heilbrunn: Klinkhardt.

Kleinknecht, M., Bohl, T., Maier, U. \& Metz, K. (2011a). Aufgaben und Unterrichtsplanung. Jahrbuch für Allgemeine Didaktik, 1(1), 59-75.

Kleinknecht, M., Maier, U., Metz, K. \& Bohl, T. (2011b). Analyse des kognitiven Aufgabenpotenzials. Entwicklung und Erprobung eines allgemeindidaktischen Auswertungsmanuals. Unterrichtswissenschaft, 29(4), 329-345.

Klieme, E. (2018). Unterrichtsqualität. In M. Gläser-Zikuda, M. Harring \& C. Rohlfs (Hrsg.), Handbuch Schulpädagogik, (S. 393-408). Münster: Waxmann.

Klieme, E. \& Rakoczy, K. (2008). Empirische Unterrichtsforschung und Fachdidaktik. Outcome-orientierte Messung und Prozessqualität des Unterrichts. Zeitschrift für Pädagogik, 54(2), 222-237.

Klieme, E., Schümer, G. \& Knoll, S. (2001). Mathematikunterricht in der Sekundarstufe I: „Aufgabenkultur“ und Unterrichtsgestaltung. In Bundesministerium für Bildung und Forschung (Hrsg.), TIMSS - Impulse für Schule und Unterricht. Forschungsbefunde, Reforminitiativen, Praxisberichte und Video-Dokumente, (S. 4358). Bonn: BMBF.

Kühn, S. M. (Hrsg.). (2010). Steuerung und Innovation durch Abschlussprüfungen? Wiesbaden: VS-Verlag.

Kunter, M., Baumert, J., Blum, W., Klusmann, U., Krauss, S. \& Neubrand, M. (Hrsg.). (2011). Professionelle Kompetenz von Lehrkräften. Ergebnisse des Forschungsprogramms COACTIV. Münster: Waxmann.

Leisen, J. (2006). Aufgabenkultur im mathematisch-naturwissenschaftlichen Unterricht. Der mathematische und naturwissenschaftliche Unterricht, 59(5), 260-266.

Lipowsky, F. (2015). Unterricht. In E. Wild \& J. Möller (Hrsg.), Pädagogische Psychologie, (S. 69-105). Berlin, Heidelberg: Springer.

Mayer, R.E. (2004). Should there be a three-strikes rule against pure discovery learning? The case for guided methods of instruction. American Psychologist, 59(1), $14-19$. 
Minnameier, G., Hermkes, R. \& Mach, H. (2015). Kognitive Aktivierung und Konstruktive Unterstützung als Prozessqualitäten des Lehrens und Lernens. Zeitschrift für Pädagogik, 61(6), 837-856.

Moosbrugger, H. \& Kelava, A. (Hrsg.). (2012). Testtheorie und Fragebogenkonstruktion. Berlin u. a.: Springer.

Neubrand, J. (Hrsg.). (2002). Eine Klassifikation mathematischer Aufgaben zur Analyse von Unterrichtssituationen. Selbsttätiges Arbeiten in Schülerarbeitsphasen in den Stunden der TIMSS-Video-Studie. Hildesheim: Franzbecker.

Neubrand, M., Jordan, A., Krauss, S., Blum \& Löwen, K. (2011). Aufgaben im COACTIV-Projekt: Einblicke in das Potenzial für kognitive Aktivierung im Mathematikunterricht. In M. Kunter, J. Baumert, W. Blum, U. Klusmann, S. Krauss, \& M. Neubrand (Hrsg.), Professionelle Kompetenz von Lehrkräften. Ergebnisse des Forschungsprogramms COACTIV, (S. 115-132). Münster: Waxmann.

Nickolaus, R. \& Wuttke, E. (2010). Ergebnisse und Desiderata zur Unterrichtsforschung in der beruflichen Bildung. In R. Nickolaus, G. Pätzold, H., Reinisch, T. Tramm (Hrsg.), Handbuch Berufs- und Wirtschaftspädagogik, (S. 168-173). Bad Heilbrunn: Klinkhardt.

Praetorius, A.-K., Klieme, E., Herbert, B. \& Pinger, P. (2018). Generic dimensions of teaching quality: The German framework of the three basic dimensions. ZDM Mathematics Education, 50(3), 407-426.

Praetorius, A.-K., Pauli, C., Reusser, K., Rakoczy, K. \& Klieme, E. (2014). One lesson is all you need? Stability of instructional quality across lessons. Learning and Instruction, 31(1), 2-12.

Reiber, K. (2010). Empirische Befunde zur Unterrichtskultur in der Pflegeausbildung. Bildungsforschung, 7(1), 118-131.

Renkl, A. (2015). Unterricht. In E. Wild \& J. Möller (Hrsg.), Pädagogische Psychologie, (S. 3-24). Berlin, Heidelberg: Springer.

Remmers, H. (2011). Pflegewissenschaft als transdisziplinäres Konstrukt - Einleitung. In H. Remmers. (Hrsg.), Pflegewissenschaft im interdisziplinären Dialog, (S. 747). Göttingen: V\&R Unipress.

Rumann, S. \& Tiemann, R. (2007). Aufgabeneinsatz und Kompetenzvermittlung im Chemieunterricht. In D. Höttecke (Hrsg.), Gesellschaft für Didaktik der Chemie und Physik: Naturwissenschafticher Unterricht im internationalen Vergleich, (S. 136-138). Münster: Lit-Verlag.

Schabram, K. (2007). Lernaufgaben im Unterricht: Instruktionspsychologische Analysen am Beispiel der Physik. Online: https://duepublico.uni-duisburg-essen.de/servlets/DocumentServlet?id=17440 (01.10.18).

Scheidt-Nave, C., Richter, S., Fuchs, J. \& Kuhlmey, A. (2010). Herausforderungen an die Gesundheitsforschung für eine alternde Gesellschaft am Beispiel „Multimorbidität". Bundesgesundheitsblatt, 53(5), 441-450.

Seifried, J. \& Wuttke, E. (2015): Was wissen und können (angehende) Lehrkräfte an kaufmännischen Schulen? - Empirische Befunde zur Modellierung und Messung der professionellen Kompetenz von Lehrkräften. In S. Schumann, F. Eberle (Hrsg.), Ökonomische Kompetenzen in Schule, Ausbildung und Hochschule. Empirische Pädagogik, 29(1), Themenheft. Landau, 125-145. 
Walter, A. (2015). Die hochschuldidaktische Arbeit mit authentischen Fällen in berufsbegleitenden Studiengängen. In B. Klages, M. Bonillo, S. Reinders, A. Bohmeyer (Hrsg.), Gestaltungsraum Hochschullehre. Potenziale nicht-traditionell Studierender nutzen, (S. 193-210). Opladen u. a.: Budrich.

Wendt, H., Bos, W., Selter, C., Köller, O., Schwippert, K. \& Kasper D. (Hrsg.). (2016). TIMSS 2015. Mathematische und naturwissenschaftliche Kompetenzen von Grundschulkindern in Deutschland im internationalen Vergleich. Münster: Waxmann.

Wesselborg, B. (2016). Videobasierte Unterrichtsforschung: Einführung und Perspektiven zur Erforschung von Lehr-Lern-Prozessen am Beispiel der Pflegeausbildung. In E. Brinker-Meyendriesch \& F. Arens (Hrsg.), Diskurs Berufspädagogik Pflege und Gesundheit, (S. 114-131). Berlin: WVB.

Wesselborg, B. \& Bögemann-Großheim, E. (2017). Bundesländerspezifische Regelungen für die hauptberufliche Lehrtätigkeit an Gesundheits- und Krankenpflegeschulen in Deutschland - eine Ist-Analyse. PADUA, 12(2), 109-116.

Weyland, U. \& Reiber, K. (2013). Lehrer/-innen-Bildung für die berufliche Fachrichtung Pflege in hochschuldidaktischer Perspektive. In U. Faßhauer, B. Fürstenau \& E. Wuttke (Hrsg.), Jahrbuch der berufs-und wirtschaftspädagogischen Forschung 2013, (S. 189-202). Opladen u. a.: Budrich.

Weyland, U. \& Reiber, K. (Hrsg.). (2017). Entwicklungen und Perspektiven in den Gesundheitsberufen - aktuelle Handlungs- und Forschungsfelder. Bielefeld: Bertelsmann.

Wirtz, M. \& Caspar, F. (2002). Beurteilerübereinstimmung und Beurteilerreliabilität. Methoden zur Bestimmung und Verbesserung der Zuverlässigkeit von Einschätzungen mittels Kategoriensystemen und Ratingskalen. Göttingen: Hogrefe. 


\title{
Entwicklung und Validierung eines Schemas zur Evaluation von Serious Games im Kontext von Financial Literacy
}

\author{
Julia Schultheis und Carmela Aprea
}

\section{Problemstellung und Zielsetzung}

Financial Literacy - hier verstanden als die Fähigkeit, adäquat mit Geld- und Finanzangelegenheiten umzugehen - ist bereits seit einiger Zeit im Fokus der bildungspolitischen und wissenschaftlichen Diskussion (Aprea 2012). Es gibt eine Vielzahl von Gründen für diese verstärkte Diskussion, wie beispielsweise die globalen und nationalen Wirtschaftskrisen oder die steigende Gefahr von Schuldenfallen durch stetig zunehmende Komplexität von Finanzprodukten.

Trotz der anerkannten Bedeutsamkeit des Themas und einer daraus resultierenden Vielzahl von Bemühungen, Financial Literacy zu messen und mittels Finanzbildungsprogrammen zu fördern, zeigen Meta-Analysen (z. B. Fernandes, Lynch \& Netemeyer 2014), dass diese Bemühungen nicht so erfolgreich sind, wie sie sein könnten und müssten. Dabei ist zu vermuten, dass dies auch an der Vernachlässigung von Verhaltenskomponenten und motivationalen sowie sozio-emotionalen Aspekten sowohl beim Assessment als auch bei Interventionen zur Förderung von Financial Literacy liegt (z. B. Aprea \& Wuttke 2016).

Eine Möglichkeit, den genannten Schwächen zu begegnen, wird im Einsatz von Serious Games gesehen. Serious Games werden hier verstanden als "digital games that merge a non-entertaining purpose (serious) with a video game structure (games)" (Djaouti, Alvarez \& Jessel 2011, 120). Wie etwa Wouters, van Nimwegen, van Oostendorp und van der Spek (2013) darlegen, haben Serious Games das Potenzial, domänenspezifische Problemlösefähigkeit und Interesse sowie intrinsische Motivation zu fördern. Die Analysen dieser Autoren zeigen jedoch auch, dass sich dieses Potenzial nicht als Selbstläufer entfaltet, sondern sowohl an eine gute didaktische Gestaltung der Games als auch an eine gute Integration in eine Lernumgebung oder ein Assessment gekoppelt ist.

Der potenzielle Nutzen von Serious Games wurde in den vergangenen Jahren auch im Kontext von Financial Literacy erkannt, und es wurden zahlreiche Games in diesem Kontext entwickelt. Allerdings fehlt es bislang an einer Evaluation, ob und inwiefern sich diese Spiele für didaktische Zwecke eignen. An dieser Stelle setzt die im vorliegenden Beitrag vorgestellte Studie an, deren 
Ziel die Entwicklung und Validierung eines Evaluationsschemas für Serious Games im Kontext von Financial Literacy ist. Die Studie ist Teil eines größeren Forschungsprojekts, bei dem es zunächst darum geht, vorhandene Games anhand des entwickelten Schemas im Rahmen einer Inputevaluation zu analysieren und zu bewerten. Die als gut befundenen Spiele sollen sodann einer Prozess- und Outcome-Evaluation unterzogen werden.

Der Beitrag ist wie folgt aufgebaut: Im folgenden Abschnitt wird zunächst der konzeptuelle Rahmen des Projekts sowie insbesondere der hier thematisierten Studie dargestellt, und es wird anschließend über das methodische Vorgehen bei der Entwicklung und Validierung des Evaluationsschemas berichtet (Abschnitt 3). Im Ergebnisteil (Abschnitt 4) wird anschließend das finale Schema präsentiert und dessen Anwendung anhand eines exemplarischen Financial Literacy Spiels verdeutlicht. Der Beitrag endet mit einem kurzen Fazit und Ausblick auf die nächsten Forschungsschritte (Abschnitt 5).

\section{Konzeptueller Rahmen}

Zur Fundierung des Gesamtprojekts wird das „Input-Process-Outcome Model“ von Garris, Ahlers und Driskell (2002) herangezogen. Dieses Modell basiert auf der Grundannahme, dass Serious Games das Potenzial haben, bei Lernenden selbstbestimmtes Verhalten zu fördern. Dieses Potenzial entsteht laut Garris et al. (2002) durch die Struktur und Funktionsweise von Games. Die Struktur besteht aus den Dimensionen Lerninhalte und Spieleigenschaften (Input). Bei der Exploration der Funktionsweise beim Spielen kommt es zu einem Kreislauf (Game Cycle) aus dem Verhalten der Spielenden, den Rückmeldungen des Spiels und einer Beurteilung des Games. Durch das mehrfache Durchlaufen dieses Kreislaufs werden Kompetenzen erworben (Process). Im letzten Teil des Modells ist ein Debriefing vorgesehen, um die Inhalte auf die Realität zu übertragen und den Lernerfolg zu sichern (Outcome). 


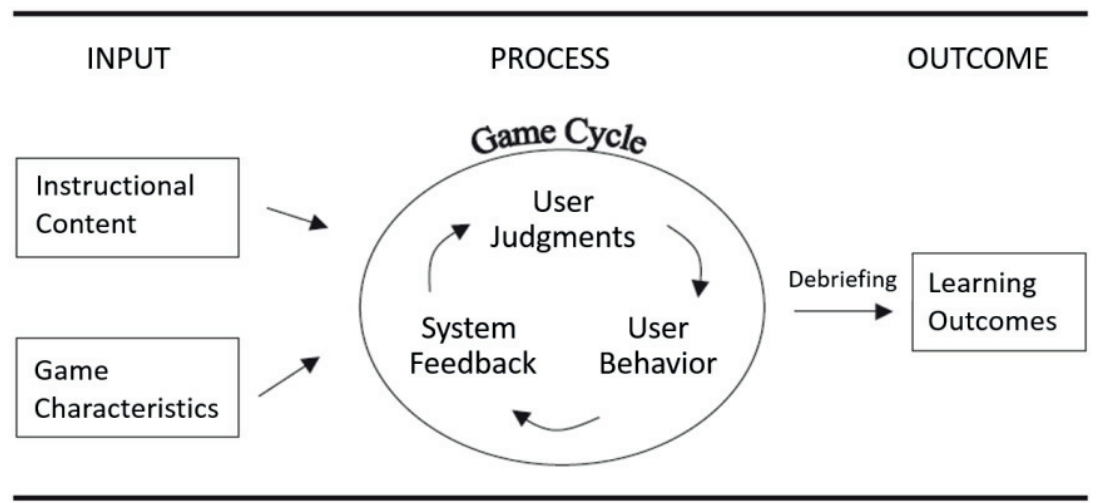

Quelle: Garris et al. 2002, 445.

Die vorliegende Studie fokussiert auf die Inputseite dieses Modells und damit auf die Lerninhalte und Spieleigenschaften, die für die Entwicklung eines Evaluationsschemas weiter konkretisiert werden sollen. Dabei erfordert die Konkretisierung der Lerninhalte eine Konzeptualisierung von Financial Literacy, während die Konkretisierung der Spieleigenschaften mittels mediendidaktischer Gestaltungsaspekte des Game-Designs erfolgen soll. Auf beide Dimensionen wird im Folgenden eingegangen.

(1) Konzeptualisierung von Financial Literacy. Betrachtet man die Literatur zur Konzeptualisierung von Financial Literacy, so fällt eine große Bandbreite an Zugängen auf, welche von einer wissensorientierten Auffassung bis hin zu einer kompetenzorientierten Auffassung reicht (Aprea \& Wuttke 2016). Eine wissensorientierte Konzeptualisierung von Financial Literacy ist am weitesten verbreitet. Sie findet sich beispielsweise in den prominenten „Big 3“ Fragen von Lusardi und Mitchell (2011), welche im Single-Choice Format die Bereiche Zinsberechnung, Auswirkungen von Inflation und Risikodiversifizierung abdecken. Problematisch am Einsatz dieser Fragen als diagnostisches Instrument ist indes, dass sie vorrangig auf mathematisches Grundverständnis (Numeracy) und einen sehr einschränkten Bereich domänenspezifischen Wissens abzielen. Folglich lassen sie nur in begrenztem Maße didaktische Schlussfolgerungen zu. Demgegenüber werden Finanzentscheidungen - und somit das Verhalten im Umgang mit Geld - nicht berücksichtigt.

$\mathrm{Da}$ es im vorliegenden Forschungskontext um eine didaktische Nutzung von Serious Games geht, welche insbesondere die Verhaltensdimension angemessen einbeziehen sollte, wird zur Konkretisierung der Inhaltsseite von Games auf eine kompetenzorientierte Konzeptualisierung von Fiancial Literacy rekurriert. Angelehnt an die Kompetenzdefinition von Weinert (2001) verste- 
hen wir hierunter ein Potenzial, welches Personen dazu befähigt, Finanzentscheidungen effektiv planen, durchführen und kontrollieren zu können. Dieses Potenzial beruht nicht nur auf (domänenspezifischem und -übergreifendem) Wissen, sondern ebenso auf Einstellungen, Motivationen und Werthaltungen im Umgang mit Geld. Financial Literacy im Sinne dieser Konzeptualisierung wurde im Rahmen eines Vorläuferprojekts in die Kompetenzdimensionen „persönliches Finanzmanagement“" und „ökonomischer und gesellschaftlicher Kontext" (auch systemische Ausrichtung) ausdifferenziert (vgl. Aprea, Wuttke, Leumann \& Heumann 2015). Dieses Kompetenzmodell wird im Folgenden als Inhaltsmodell herangezogen, da es die Facetten von Financial Literacy in ihrer ganzen Bandbreite umfasst und verdeutlicht. Das persönliche Finanzmanagement bezieht sich auf Finanzentscheidungen im Privatleben. Es lassen sich die folgenden Facetten unterscheiden: Geld verdienen/einnehmen, die eigenen Einnahmen einschätzen, Ausgaben in Abstimmung mit den eigenen Bedürfnissen und Möglichkeiten planen, kurzfristige Geldreserven anlegen, Budget aufstellen und überprüfen, mit Kreditkarten umgehen, Steuererklärung ausfüllen, Geld ausgeben, Ver- und Überschuldung vermeiden, Bankund Finanzdienstleistungen des täglichen Bedarfs nutzen, Geld sparen/Vermögen aufbauen, Geld leihen/Kredit aufnehmen/Finanzierungsarten, Altersvorsorge treffen, Versicherungen vergleichen/abschließen und Informations- und Beratungsangebote im Kontext von Geld- und Finanzangelegenheiten kennen. Die systemische Ausrichtung bezieht sich auf die ökonomischen und politischen Rahmenbedingungen, innerhalb derer die Finanzentscheidungen getroffen werden. Die folgenden Facetten werden dabei unterschieden: Geld- und finanzpolitische Zusammenhänge, realwirtschaftliche Zusammenhänge, generell gesamtökonomische Zusammenhänge, Wissen über finanzbezogene Fakten, Rahmenbedingungen des politischen Systems, System der Sozialversicherung und Steuerwesen (zur konzeptuellen und empirischen Herleitung dieser Facetten siehe Aprea et al. 2015).

(2) Gestaltungsprinzipien des Game-Designs. Damit Serious Games ihre Potenziale für Motivation und Lernen entfalten können, müssen sie verschiedene Gestaltungsprinzipien erfüllen. In der einschlägigen Literatur (z. B. Garris et al. 2002; Petko, 2008; Kerres, Bormann \& Vervenne 2009) lassen sich insbesondere die folgenden Gestaltungsprinzipien identifizieren:

- Situiertheit: Die Möglichkeit von situiertem Lernen ergibt sich in Spielen durch die Schaffung einer fiktiven Spielwelt. Die Spielenden können in diese Spielwelt eintauchen und in Situationen handeln. Da die Konsequenzen ihres Handelns keine Auswirkungen auf die Realität haben, wird dadurch ein Probehandeln ermöglicht. Die Spielenden bekommen durch das Spiel ein Feedback auf ihr Handeln, sowohl durch Erfolg oder Misserfolg im Spiel, als auch durch ins Spiel integrierte Rückmeldungen. Die Struktur der Spielhandlungen und des Feedbacks sollte in Beziehung mit den zu erlernenden Zielhandlungen stehen. 
- Ziel- und Regelorientierung: In der Spielwelt sind Ziele definiert und Regeln vorgegeben. Diese geben dem Spiel eine Struktur und bilden den Rahmen für Handlungs- und Entscheidungsmöglichkeiten der Spielenden. Auch wenn das Ziel klar definiert ist, sollten für das Erreichen eines Ziels verschiedene Lösungswege möglich sein.

- (zunehmende) Herausforderung: Die Spielenden sammeln Erfahrung und Wissen in der Auseinandersetzung mit dem Game. Um eine Verringerung der Motivation zu vermeiden, sollten die zu lösenden Aufgaben im Spiel an Komplexität zunehmen.

- Erlernbarkeit der Spielmechanismen: Zu den Spielmechanismen gehört die Komplexität der Spielsteuerung. Diese sollten leicht zu erlernen sein, sodass nicht die Fähigkeiten beim Gamen (z. B. Geschicklichkeit) im Vordergrund stehen, sondern die tatsächliche Auseinandersetzung mit den Inhalten. Um die Spielmechanismen zu erlernen, können Tutorials oder Erklärvideos eingesetzt werden.

- Kontrolle: Durch die Kontrolle über die Spielhandlungen werden die Grundbedürfnisse Autonomieerleben und Kompetenzerleben gefördert. Diese gelten als Bedingungsfaktoren intrinsischer Motivation (Deci \& Ryan 1985). Die Befriedigung der motivationalen Grundbedürfnisse sollte durch Entscheidungen über Strategien und das selbstständige Finden von Lösungswegen ermöglicht werden.

- Soziale Interaktionsmöglichkeiten: Zur Förderung von sozialem Lernen ist die Implementierung von sozialen Interaktionsmöglichkeiten in einem Game wünschenswert. Diese sollten beispielsweise durch einen Multiplayer-Modus oder eine Turnbasierung in ein Spiel integriert sein.

\section{Methodisches Vorgehen}

Basierend auf den vorangehend skizzierten konzeptuellen Überlegungen erfolgte die Entwicklung und Validierung des Evaluationsschemas im Rahmen eines am Design-Based Research (z. B. DBRC 2003) angelehnten Prozesses, der mehrere Phasen des (Re-)Designs und der Prüfung des Schemas umfasste, und innerhalb dessen auch potenzielle Nutzer und Anwender der Games bzw. weitere Stakeholder eingebunden waren. Es wurden die folgenden Schritte durchlaufen:

1. Zunächst wurde eine zweistufige Bedarfsanalyse durchgeführt. Um einen ersten Überblick zu gewinnen, welche Kriterien in das Evaluationsschema inkludiert werden sollten, wurde zum einen eine systematische Suche nach 
bestehenden Rastern und Schemata zur Analyse und Bewertung von digitalen Spielen über Google Scholar und anschließend per Schneeballverfahren durchgeführt. Die Suche bezog sich ausschließlich auf Analyseraster aus dem Bereich Instruktionsdesign und Assessment. Es wurden acht Raster ausgewählt und ausgewertet. Ergänzend zu dieser Auswertung wurden zum anderen Interviews mit Eltern, Lehrpersonen und Experten aus der Medienpädagogik und dem Bereich Finance geführt, um herauszufinden, was diesen betroffenen Gruppen beim Einsatz von digitalen Spielen im Kontext von Financial Literacy wichtig ist.

2. Auf der Basis der im vorangegangenen Abschnitt skizzierten konzeptuellen Überlegungen sowie der Auswertung der vorhandenen Raster und der Interviews wurde ein erster Kriterienkatalog entwickelt und auf seine Praktikabilität bzw. Verständlichkeit geprüft. Zu diesem Zweck haben vier Codierer zwei Spiele unabhängig voneinander anhand des Kriterienkatalogs ausgewertet. Im Falle von abweichenden Einschätzungen wurden diese anschließend unter den Codierern diskutiert und es wurden gemeinsam Vorschläge für Verbesserungen des Kriterienkatalogs bzw. dessen Handhabung gemacht (konsensuelle Validierung).

3. Die aus dem vorangehenden Schritt gewonnenen Erkenntnisse wurden in eine zweite Version des Kriterienkatalogs eingearbeitet, welcher einer erneuten Überprüfung unterzogen wurde, diesmal mit weniger Codierern $(\mathrm{N}=2)$ und einer höheren Anzahl an Games $(\mathrm{N}=10)$, um eine größere Diversität von Spielen abzudecken. Zur Erfassung der Übereinstimmung der Auswertungsergebnisse wurde die Interraterreliabilität ermittelt.

Die Ergebnisse des hier skizzierten methodischen Vorgehens werden im nächsten Schritt vorgestellt. Darüber hinaus wird die Anwendung der Kriterien an einem Beispiel verdeutlicht.

\section{Ergebnisse}

\subsection{Ergebnisse der Bedarfsanalyse}

Die Analyse der Raster und Schemata ergab, dass die Zusammenhänge von spielerischen und pädagogischen Elementen eine zentrale Rolle spielen (Amory 2006; Arnab et al. 2015; Carvalho et al. 2015). Um diese Zusammenhänge zu analysieren, werden Kriterien wie Lernziele, Lernaufgaben im Spiel und die Spielgeschichte herangezogen. In der Mehrzahl der Analysesysteme werden Tabellen zur Analyse eingesetzt (de Freitas \& Oliver 2006; Foster, Mishra \& Koehler 2011; Rodriguez-Hoyos \& Gomes 2012). In drei Fällen 
wird zusätzlich noch eine graphische Darstellung der Spiele gewählt, da so das Verständnis des Spielaufbaus einfacher verdeutlicht werden kann und eine Analyse von komplexen Spielelementen erleichtert wird (Amory 2006; Arnab et al. 2015; Carvalho et al. 2015). Keines der vorhandenen Raster war im Kontext von Financial Literacy entwickelt worden, sodass eine entsprechende Ergänzung der Raster im Hinblick auf die Inhaltsdimension, also die Kompetenzfacetten von Financial Literacy, erforderlich war. Weiterer Ergänzungsbedarf ergab sich zudem aus den Interviews, welche zeigten, dass es sowohl den Eltern als auch den Lehrpersonen wichtig ist, dass in einem Spiel, welches zu Lernzwecken eingesetzt werden soll, keine Gewalt oder Diskriminierung vorkommen sollte. Außerdem wiesen die Lehrpersonen darauf hin, dass viele Spiele von Versicherungsgesellschaften oder Banken finanziert würden. Die Lehrpersonen legten hier besonderen Wert auf Spiele ohne Werbung von diesen Unternehmen. Von Expertenseite wurde darauf hingewiesen, dass nicht alle Schüler*innen zu Hause spielen und daher nicht alle ein Grundverständnis für Gamemechanismen hätten. Daher sollte ein Spiel für die Förderung und das Assessment von Financial Literacy leicht zu bedienen sein, um nicht jene Lernenden zu bevorteilen, die in ihrer Freizeit spielen.

\subsection{Ergebnisse der Entwicklung und ersten Prüfung der Kriterien}

Basierend auf den Gestaltungsprinzipien des Game-Designs sowie den Ergebnissen der Analyse der Literatur und der Interviews wurde ein erster Kriterienkatalog erstellt. Dieser teilt sich in vier Kriteriengruppen: Kontextuelle Kriterien, ethische Kriterien, Game-Design Kriterien und curriculare Kriterien (siehe Abb. 1). Bei den kontextuellen Kriterien handelt es sich um Informationen, die durch eine Recherche über ein Spiel gewonnen werden können, wie beispielsweise die Zielgruppe oder die technischen Anforderungen eines Spiels. Durch diese Informationen können Akteure, die ein Game einsetzen wollen, bereits eine erste Vorauswahl treffen, ob das gewählte Spiel für den Zweck passend ist. Ethische Kriterien beziehen sich vor allem auf die in den Interviews geäußerten Anliegen der Lehrpersonen und Eltern. Sie geben an, ob ein Spiel frei von Gewalt und Werbung ist, und ob einzelne Nutzergruppen systematisch diskriminiert werden. Die kontextuellen und ethischen Kriterien bilden den Rahmen des Schemas und geben Auskunft über die grundsätzliche Eignung eines Spiels für die Verwendung in didaktischen Kontexten, ohne auf dessen inhaltliche und mediale Ausgestaltung einzugehen.

Mit den Game-Design Kriterien wird ein Spiel im Hinblick auf die medialen Gestaltungselemente wie beispielsweise die Spielstory, das Spielgenre, den Spielaufbau, die Hilfestellungen im Spiel und den Schwierigkeitsgrad der Spielsteuerung untersucht. Diese Kriterien operationalisieren insbesondere die oben genannten Gestaltungsprinzipien an das Game-Design. Die Situiertheit 
findet sich in den Kriterien Narrativ, Spielaufgaben und Charaktere wieder. Durch die Erfassung des Spielziels wird die Regel- und Zielorientierung abgebildet. Die zunehmende Herausforderung wird durch die Spielaufgaben operationalisiert. In den Kriterien Hilfestellungen und Schwierigkeitsgrad der Spielmechanismen findet sich die Erlernbarkeit der Spielmechanismen wieder. Das Prinzip der Kontrolle wird sowohl über die Spielstruktur als auch über die Spielaufgaben operationalisiert. Soziale Interaktionsmöglichkeit wird in einem eigenständigen Kriterium abgebildet. Die curricularen Kriterien umfassen neben den Lerninhalten im Sinne der abgebildeten Kompetenzfacetten bzw. Finanzentscheidungen auch die Lernziele sowie die Lernaufgaben. Die curricularen Kriterien sind im Kontext von Serious Games mit den Game-Design Kriterien verknüpft, da beispielsweise die einzelnen Aufgaben im Spiel in der Regel auch eine Lernaufgabe beinhalten.

Zur Auswertung der Spiele wurden die Kriterien in eine Tabellenform überführt, deren Zellen die Auswerter im Freitext ausfüllen sollten.

Abb. 2: Verhältnis der vier Hauptkriteriengruppen

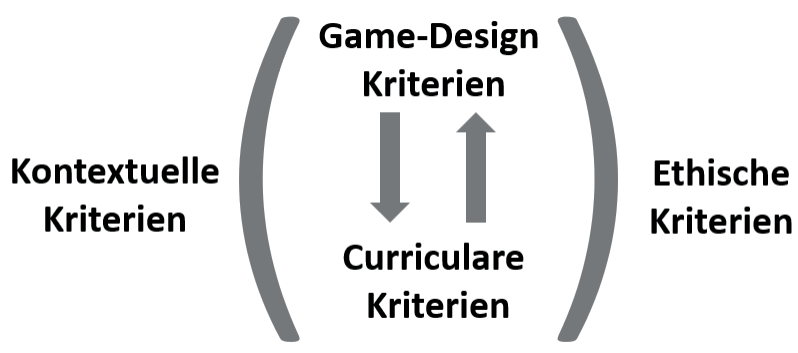

Quelle: eigene Abbildung

Die erste Überprüfung der Kriterien ergab zum einen, dass die im Freitext auszufüllende Tabelle die Codierer nicht hinreichend darin unterstützte, die Kriterien umfänglich und genau anzuwenden, was in der Folge zu einer hohen Divergenz der Auswertungsergebnisse führte. Diese Schwierigkeit trat vor allem bei den Kriterien Lernziele, Schwierigkeitsgrad der Spielsteuerung und Spielgenre auf. Außerdem fiel es den Codierern nicht immer leicht, die Handlungen im Spiel eindeutig den Kompetenzfacetten von Financial Literacy zuzuordnen. 


\subsection{Ergebnisse der Überarbeitung und zweiten Prüfung der Kriterien}

Um das Problem der ungenauen Anwendung von Kriterien zu lösen, wurde jedem Kriterium eine oder mehrere Leitfragen zugeordnet. Ferner wurde ein Flowchart entwickelt, mit dem ein Spiel in einzelne Schritte zerlegt werden kann. Hierdurch wird die Zuordnung der Spielhandlung zu den Kompetenzfacetten erleichtert.

Bei der Überprüfung der zweiten Fassung des Kriterienkatalogs zeigte sich, dass mit diesen Maßnahmen die Handhabung und Eindeutigkeit des Schemas verbessert werden konnte. Die Zuordnung der Kompetenzfacetten bereitete nun kaum noch Schwierigkeiten. Auch die Leitfragen trugen zu einer Vereinheitlichung der Anwendung und Interpretation der Kriterien bei. Lediglich bei den Kriterien „Spielgenre“ und „Schwierigkeitsgrad der Spielsteuerung“ waren weiterhin Probleme bei der eindeutigen Anwendung zu verzeichnen, sodass hier eine Standardisierung der Einschätzungen zu diesen Kriterien eingefügt wurde. Zur anschließenden Prüfung der überarbeiteten Fassung wurden die folgenden Spiele herangezogen: Finance Mission Heroes, Bite Club, Farm Blitz, Cure Runners, Moneytopia, Spent, Gen-i-Revolution, Financial Football, Groove Nation und Mad Money. Insgesamt lag die Interraterreliabilität der beiden Codierer bei .773, was als akzeptabel angesehen werden kann (Bortz \& Döring 2006).

\subsection{Exemplarische Anwendung der Kriterien}

Zur Verdeutlichung des Schemas wird dieses im Folgenden auf das Serious Game „Celebrity Calamity“ angewendet. Celebrity Calamity eignet sich zur Demonstration zum einen aufgrund der transparenten Spielstruktur und zum anderen wegen der Vielfalt der abgebildeten Finanzentscheidungen. In dem Spiel assistieren Spielende einer prominenten Person und haben die Aufgabe, sich um deren finanzielle Angelegenheiten zu kümmern, wie z. B. Einkäufe zu erledigen und die Kreditkartenrechnung zu begleichen.

Die Entwicklung des Spiels wurde von einer durch verschiedene US-amerikanischen Finanzinstitutionen gegründeten Stiftung (Commonwealth) finanziert. Es ist sowohl als Browsergame als auch als App kostenfrei verfügbar. Als Zielgruppe werden Frauen im Alter von 18 bis 35 Jahren angegeben. Frauen in diesem Alter sind laut der für die Entwicklung verantwortlichen Institution besonders gefährdet, durch eine Kreditkarte in eine Schuldenfalle zu geraten, da die Hemmschwelle zum Geldausgeben gesenkt wird und leicht der Überblick über das eigene Budget verloren gehen kann. 
Tab. 1 Ausprägung der kontextuellen Kriterien beim Spiel Celebrity Calamity

\begin{tabular}{|l|l|l|}
\hline \multirow{4}{*}{} & Herausgeber & Commonwealth (früherer Name: D2D Fund) \\
\cline { 2 - 3 } & Entwickler & Keine Angabe \\
\cline { 2 - 3 } & Finanzierung & Commonwealth (früherer Name: D2D Fund) \\
\cline { 2 - 3 } & Distributionskanal & Internet (https://financialentertainment.org/ \\
\multirow{2}{*}{} & & celebritycalamity) und verfügbar als Apps \\
& & für iOS und Android \\
\cline { 2 - 3 } & Technische Anforderungen & Browsergame (Adobe Flash Player), Maus \\
& & und Tastatur \\
\cline { 2 - 3 } & Kosten & Keine Anschaffungskosten \\
\cline { 2 - 3 } & Sprachen & Englisch, Spanisch \\
\cline { 2 - 3 } & Zielgruppe & Frauen in der Altersklasse von 18-35 Jahren \\
\hline
\end{tabular}

Quelle: eigene Abbildung

Bezüglich der ethischen Kriterien zeigt sich, dass bei „Celebrity Calamity“ weder Gewalt noch Werbung vorkommen. Bei der Nutzung des Spiels sind keine kostenpflichtigen Extras notwendig. Auch wenn im Spiel keine systematische Diskriminierung erkennbar ist, könnte es sein, dass das Spiel für männliche Spieler aufgrund der Spielstory weniger interessant ist.

Tab. 2: Ausprägung der ethischen Kriterien beim Spiel Celebrity Calamity

\begin{tabular}{|c|c|c|}
\hline \multirow{4}{*}{ 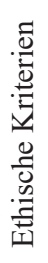 } & Abwesenheit von Gewalt & Im Spiel gibt es keinerlei Gewalt. \\
\hline & Werbung & Keine Werbung vorhanden. \\
\hline & In-Game Käufe & $\begin{array}{l}\text { Es sind keine kostenpflichtigen In-Game } \\
\text { Käufe möglich oder notwendig. }\end{array}$ \\
\hline & $\begin{array}{l}\text { Fairness bezüglich der } \\
\text { Nutzergruppen }\end{array}$ & $\begin{array}{l}\text { Das Spiel zielt insbesondere auf Frauen im Al- } \\
\text { ter zwischen } 18 \text { und } 35 \text { ab. Männliche Spieler } \\
\text { könnten den Plot wenig ansprechend finden. }\end{array}$ \\
\hline
\end{tabular}

Quelle: eigene Abbildung

Die Homepage zum Spiel „Celebrity Calamity“ nennt vier explizite Lernziele, die sich alle auf den Umgang mit Kreditkarten und die Bezahlung der Kreditkartenrechnung beziehen. Als Lernaufgaben sind zum einen die Entscheidung über die Verwendung von Kredit- oder Debitkarten und zum anderen die Begleichung der Kreditkartenrechnung zu nennen. Die abgebildeten Kompetenzfacetten werden anhand des Flow-Charts (vgl. Abb. 3) an späterer Stelle erläutert. 
Tab. 3: Ausprägung der curricularen Kriterien beim Spiel Celebrity Calamity

\begin{tabular}{|c|c|c|}
\hline \multirow{3}{*}{ 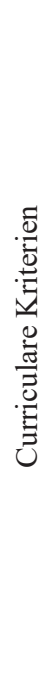 } & Lernziele & $\begin{array}{l}\text { Explizite Lernziele (übersetzt): } \\
\text { - } \quad \text { Mehr als das Minimum der Kreditkarten- } \\
\text { - } \quad \text { Krechnung bezahlen } \\
\text { - } \quad \text { Gebühren für Überziehungszinsen, ver- } \\
\text { spätete Kreditkartenzahlungen und Kre- } \\
\text { ditkartenüberziehung vermeiden } \\
\text { - } \\
\text { Effektiven Jahreszins wählen }\end{array}$ \\
\hline & Lernaufgaben & $\begin{array}{ll}\text { - } & \text { Entscheidung über die Verwendung von } \\
\text { Kreditkarten und Debitkarten } \\
\text { - } \quad \text { Kreditkartenabrechnungen begleichen }\end{array}$ \\
\hline & Kompetenzfacetten & $\begin{array}{l}\text { - } \quad \text { Geld verdienen/einnehmen } \\
\text { - } \quad \text { Ausgaben in Abstimmung mit den eige- } \\
\text { - } \quad \text { Gen Bedürfnissen planen } \\
\text { - } \quad \text { Mit Kreditkarten umgehen } \\
\text { - } \quad \text { Ver- und Überschuldung vermeiden } \\
\rightarrow \text { Siehe Abb. } 3 \text { Spalte } 3\end{array}$ \\
\hline
\end{tabular}

Quelle: eigene Abbildung

Bei der Analyse des Spiels hinsichtlich des Game-Designs ist zunächst festzustellen, dass es sich bei dem Spiel um einen Mix aus Simulations- und Actiongame handelt. Durch eine Turnbasierung bietet das Spiel Möglichkeiten für eine Interaktion von mehreren Spielenden. Ein Level dauert ca. 3 Minuten und die Gesamtspieldauer beträgt 1,5 bis 2 Stunden. Der Herausgeber empfiehlt, das ganze Spiel zu spielen, da die Aufgabe, die Finanzen der prominenten Person zu verwalten, mit jedem Level komplexer wird. Das Spiel ist so konstruiert, dass es unmöglich ist, in den höchsten Levels die Kreditkartenrechnung zu bezahlen. Die Spielenden geraten dadurch in jedem Fall in eine Schuldenfalle. Dies ist durch die Herausgeber intendiert, um die Gefahr einer Schuldenfalle zu verdeutlichen. Das Erlernen der Spielmechanismen wird durch Erklärblasen in der ersten Spielrunde erleichtert. Weiterhin ist zu allen Zeitpunkten im Spiel ein Hilfe-Button vorhanden. Der Schwierigkeitsgrad der Spielsteuerung kann als einfach eingeschätzt werden, da die intuitive Handhabung durch Point-and-Click und Tastatur keine besondere Geschicklichkeit erfordert. 
Tab. 4: Ausprägung des Games-Design Kriterien beim Spiel Celebrity Calamity

\begin{tabular}{|c|c|c|}
\hline & Spielgenre & $\bigotimes_{\text {Action/Arcade }}^{\square} \begin{array}{c}\square \\
\text { Strategie }\end{array}$ \\
\hline & Interaktionsmöglichkeiten & Turn-basiert \\
\hline & Narrativ & $\begin{array}{l}\text { Die Spielenden sind die persönlichen Assisten- } \\
\text { ten von Prominenten und müssen deren Ein- } \\
\text { käufe mit Debit- und Kreditkarten bezahlen. }\end{array}$ \\
\hline & Ziel des Spiels & $\begin{array}{l}\text { Das Hauptziel ist es, die Karriereleiter nach } \\
\text { oben zu klettern. Dies wird möglich, indem } \\
\text { man die Prominenten glücklich macht und für } \\
\text { sie Einkäufe erledigt sowie das Budget gut ver- } \\
\text { waltet. }\end{array}$ \\
\hline 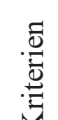 & Charaktere & $\begin{array}{l}\text { Spielende sind Assistenten der Prominenten. } \\
\text { Drei Prominente als Arbeitgeber: Alice Al- } \\
\text { budget, Ryan Buyin und Missy Moolah }\end{array}$ \\
\hline 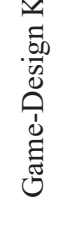 & Struktur & $\begin{array}{l}\rightarrow \text { Siehe Abb. } 3 \text { Spalte } 1 \\
\mathrm{Ab} \text { einem höheren Level ist eine Schuldenfalle } \\
\text { nicht mehr zu vermeiden. Aus dieser Schulden- } \\
\text { falle können sich die Spielenden nicht mehr be- } \\
\text { freien. Dies ist intendiert von den Spielentwick- } \\
\text { lern }\end{array}$ \\
\hline & Spielaufgaben & $\begin{array}{l}\text { Einkäufe erledigen } \\
\text { Einkäufe bezahlen } \\
\text { Kreditkartenabrechnung bezahlen } \\
\rightarrow \text { Siehe Abb. } 3 \text { Spalte } 2\end{array}$ \\
\hline & Spieldauer & $\begin{array}{l}\text { Ein Level dauert ca. 3-5 Minuten } \\
\text { Das ganze Spiel dauert ca. 1,5-2 Stunden }\end{array}$ \\
\hline & Hilfestellungen & $\begin{array}{ll}\text { - } & \text { Hilfebutton verfügbar } \\
\text { - } & \text { Funktionen werden in der ersten Runde mit } \\
& \text { Erklärblasen verdeutlicht } \\
\end{array}$ \\
\hline & $\begin{array}{l}\text { Schwierigkeitsgrad der } \\
\text { Spielsteuerung }\end{array}$ & $\begin{array}{ccc}\bigotimes_{\text {Einfach }} & \square & \square \\
\text { Mittel } & \text { Schwer }\end{array}$ \\
\hline
\end{tabular}

Quelle: eigene Abbildung

Im Flowchart (Abb. 3) werden die Schritte im Spielablauf, die Aufgaben im Spiel und die hier zu findenden Finanzentscheidungen abgebildet. Zu Beginn des Spiels wird eine prominente Person vorgestellt, für welche die Spielenden als Assistent oder Assistentin arbeiten. Die Entscheidung bezüglich des Jobs bildet die Kompetenzfacette „Geld verdienen/einnehmen“ ab. Anschließend müssen die Spielenden die Einkäufe erledigen und dabei Dinge einsammeln, die auf der Einkaufsliste stehen. Hier müssen die Spielenden abwägen, ob sie 
wirklich alles von der Einkaufsliste einsammeln und damit eventuell mehr ausgeben als sie verdienen oder nicht alles einsammeln und den Prominenten nicht zufrieden stellen. Diese Spieltätigkeit lässt sich der Facette „Ausgaben in Abstimmung mit den eigenen Bedürfnissen“ zuordnen. Beim anschließenden Bezahlen der Einkäufe muss entschieden werden, ob die Einkäufe mit einer Debitkarte oder einer Kreditkarte bezahlt werden sollen. Bei Zahlung mit Debitkarte werden die Kosten der Einkäufe direkt von den Einnahmen (Gehalt) abgezogen, während bei der Zahlung per Kreditkarte die Rechnung erst zu einem späteren Zeitpunkt beglichen werden kann. Dieser Spielhandlung lassen sich die Kompetenzfacetten ,Geld ausgeben“ und „Mit Kreditkarten umgehen“ zuordnen. Im vorletzten Schritt muss die Kreditkartenabrechnung ausgeglichen werden und die Spielenden müssen entscheiden, wie viel sie von den Kreditkartenschulden begleichen wollen ${ }^{1}$. Dabei ist auf den erhobenen Zinssatz und die anfallenden Gebühren zu achten, die entstehen können, wenn nicht der gesamte Betrag zurückgezahlt wird.

Abb. 3: Flowchart zur Darstellung des Spielablaufs und der Finanzentscheidungen im Spiel Celebrity Calamity

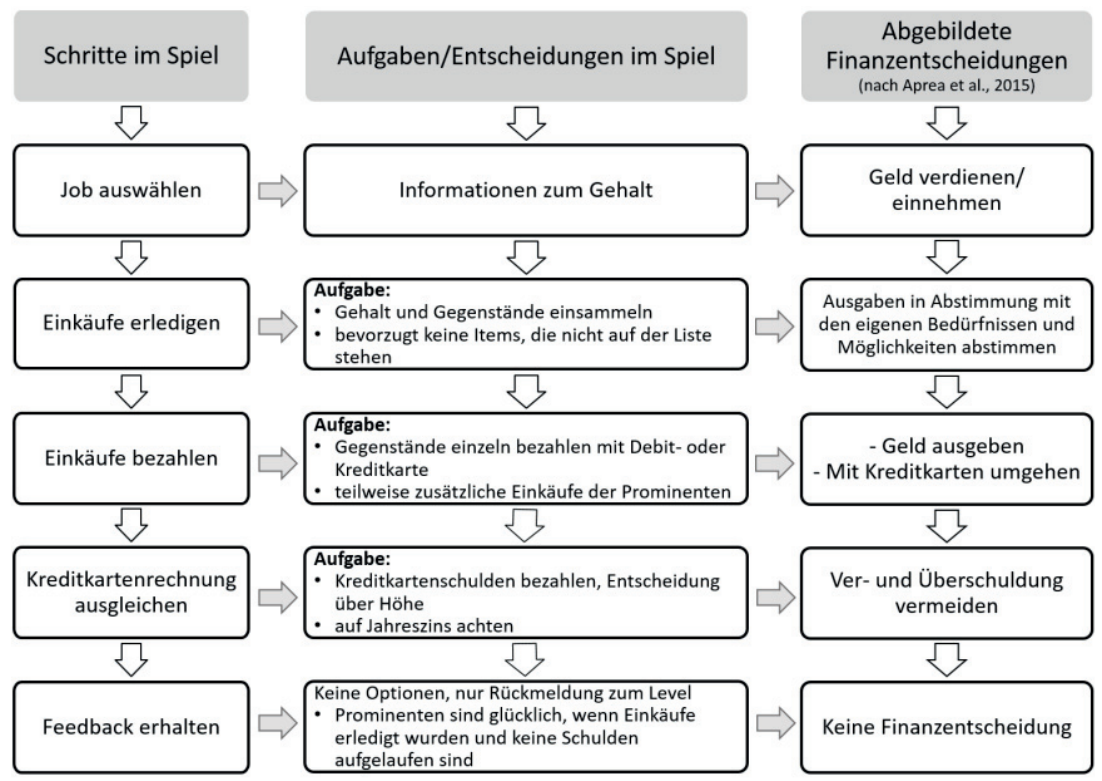

Quelle: eigene Abbildung

1 Die Möglichkeit eine Kreditkartenrechnung nur teilweise zu begleichen ist in den USA üblich, entspricht aber nicht den Gepflogenheiten in Deutschland. 
Da die Gebühren schnell ansteigen und dann nicht mehr beglichen werden können, ist die zugeordnete Finanzentscheidung „Ver- und Überschuldung vermeiden". In einem letzten Schritt bekommen die Spielenden eine Rückmeldung, ob sie ihren Prominenten zufrieden stellen konnten. Dies ist zum einen von den getätigten Einkäufen abhängig, zum anderen aber auch vom Stand der Schulden. Je höher die angehäuften Kreditkartenschulden, desto unzufriedener sind die Prominenten.

Zusammenfassend lässt sich das Spiel Celebrity Calamity damit folgendermaßen beurteilen: Durch die Spielgeschichte, die Spielaufgaben und die Charaktere wird eine Situierung des Lernens sichergestellt. Betrachtet man die Struktur des Spiels, so ist auch eine Regel- und Zielorientierung erkennbar. Das Kriterium (zunehmende) Herausforderung ist ebenfalls erfüllt, da die Schwierigkeit, alle Einkäufe zu erledigen und die Kreditkartenrechnung zu begleichen, im Laufe des Spiels ansteigt. Das Spiel ist allerdings so angelegt, dass es in den letzten Leveln unmöglich ist, die Kreditkartenrechnung zu begleichen. Laut Spielherausgeber soll dadurch ein Bewusstsein geschaffen werden, wie leicht man in eine Schuldenfalle geraten kann. In diesem Zusammenhang lässt sich hinsichtlich der Kontrolle über das Spiel feststellen, dass diese $\mathrm{zu}$ Beginn des Spiels sichergestellt ist. Aufgrund der Unmöglichkeit, die Schuldenfalle zu vermeiden, ist das Gestaltungsprinzip der Kontrolle final nicht erfüllt. Durch eine einfache Spielsteuerung ist das Erlernen der Spielmechanismen vereinfacht und schnell möglich. Es wird durch einen Hilfebutton und Erklärblasen im ersten Level erleichtert. Soziale Interaktionsmöglichkeiten sind in dem Spiel durch eine Turn-Basierung vorhanden.

\section{Fazit und Ausblick}

Der vorliegende Beitrag basiert auf der Überlegung, dass der Einsatz von Serious Games eine Möglichkeit bietet, bestehenden Schwächen beim Assessment und der Förderung von Financial Literacy wirksam zu begegnen. Dies führte zu einem zunehmenden Angebot von Serious Games in diesem Kontext. Vor diesem Hintergrund wurde über eine Studie berichtet, die auf die Entwicklung und Validierung eines Schemas zur Evaluation solcher Games abzielt. Dieses Schema soll die Grundlage für eine Inputevaluation von Serious Games im Kontext von Financial Literacy bilden. Im Anschluss an das Modell von Garris et al. (2002) geht die Studie davon aus, dass der Input durch die Dimensionen „Lerninhalte“ und „Spieleigenschaften“ erfasst werden kann. Zur weiteren Konkretisierung dieser Dimensionen wurden zum einen eine kompetenzorientierte Konzeptualisierung von Financial Literacy sowie zum anderen mediendidaktische Gestaltungsaspekte des Game-Designs herangezogen. Durch 
einen mehrstufigen Prozess der Ausarbeitung und Validierung konnte ein verständliches, praktikables und reliables Evaluationsschema entwickelt werden. Die exemplarische Anwendung am Spiel „Celebrity Calamity“ verdeutlichte das Potenzial dieses Schemas.

Die Entwicklung und Validierung des vorliegenden Schemas stellt den ersten Schritt der Inputevaluation des zu Beginn vorgestellten größeren Forschungsprojektes dar. Der nächste Schritt im Zuge der Inputevaluation ist der Einsatz des Schemas, um verfügbare Spiele im Kontext von Financial Literacy zu evaluieren. Nach ersten Recherchen handelt es sich hier um etwa 50 Spiele. Eine Auswahl der von mit Hilfe des Schemas als gut befunden Spiele soll im Rahmen von Interventionsstudien zudem weiteren Evaluationsschritten unterzogen werden. So ist im Anschluss an die Inputevaluation eine Prozessevaluation geplant, bei der der Umgang der Lernenden und Lehrenden mit Serious Games im Kontext der Finanzbildung weiter erforscht werden soll. Darüber hinaus werden einzelne Spiele in einer Outcome-Evaluation auf ihre Wirksamkeit geprüft.

Neben der Verwendung des Schemas im Rahmen des geplanten Forschungsprojekts sind weitere Einsatzmöglichkeiten denkbar. So kann das Schema beispielsweise auch herangezogen werden, um Praktiker*innen bei der Auswahl von Games für game-basierte Lernumgebungen zu unterstützen. Ferner können die Kriterien von Gamedesigner genutzt werden, um anforderungsgerechte Spiele im Bereich der Finanzbildung zu entwickeln.

\section{Literatur}

Amory, A. (2006). Game object model version II: a theoretical framework for educational game development. Educational Technology Research and Development 55, $51-77$.

Aprea, C. (2012). Messung der Befähigung zum Umgang mit Geld und Finanzthemen. Ausgewählte Instrumente und alternative diagnostische Zugänge. In Berufs- und Wirtschaftspädagogik - online, H. 22. Online: http://www.bwpat.de/ausgabe22/ aprea_bwpat22.pdf (30.03.2019).

Aprea, C., \& Wuttke, E. (2016). Financial literacy of adolescents and young adults: Setting the course for a competence-oriented assessment instrument. In C. Aprea, E. Wuttke, K. Breuer, N.K. Koh, P. Davies, B. Greimel-Fuhrmann, J.S. Lopus (eds.), International Handbook of Financial Literacy, (S. 397-414). Singapore: Springer.

Aprea, C., Wuttke, E., Leumann, S. \& Heumann, M. (2015). Kompetenzfacetten von Financial Literacy: Sichtweisen verschiedener Akteure. In S. Seeber, J. Seifried \& B. Ziegler (Hrsg.), Jahrbuch der berufs- und wirtschaftspädagogischen Forschung 2015, (S. 11-22). Leverkusen: Barbara Budrich. 
Arnab, S., Lim, T., Carvalho, M. B., Bellotti, F., de Freitas, S., Louchart, S., Suttie, N., Berta, R., \& De Gloria, A. (2015). Mapping learning and game mechanics for serious games analysis. British Journal of Educational Technology, 46, 391-411.

Bortz, J. \& Döring, N. (Hrsg.). (2006). Forschungsmethoden und Evaluation für Human- und Sozialwissenschaftler. Heidelberg: Springer.

Carvalho, M. B., Bellotti, F., Berta, R., Gloria, A. D., Sedano, C. I., Baalsrud Hauge, J., Hu, J., \& Rauterberg, M. (2015). An activity theory-based model for serious games analysis and conceptual design. Computers and Education, 87, 166-181.

de Freitas, S. \& Oliver, M. (2006). How can exploratory learning with games and simulations within the curriculum be most effectively evaluated? Computers \& Education, 46(3), 249-264.

Deci, R. M. \& Ryan, E. L. (eds.). (1985). Intrinsic Motivation and Self-Determination in Human Behavior. New York: Plenum.

Design-Based Research Collective (DBRC) (2003). Design-based research: An emerging paradigm for educational inquiry. Educational Researcher, 32(1), 5-8, 35-37.

Djaouti, D., Alvarez, J. and Jessel, J.-P. (2011). Classifying serious games: the G/P/S model. In P. Felicia (ed.), Handbook of Research on Improving Learning and Motivation Through Educational Games: Multidisciplinary Approaches, (S. 118136). Hershey, PA: IGI Global.

Fernandes, D., Lynch, J. G., \& Netemeyer, R. G. (2014). Financial literacy, financial education and downstream financial behaviors. Management Science, 60(8), 1861-1883.

Foster, A., Mishra, P. \& Koehler, M. (2011). Digital Game Analysis: Using the Pedagogical Content Knowledge Framework to Determine the Affordances of a Game for Learning. In M.S. Khine (ed.), Learning to play: exploring the future of learning with video games, (S. 189-212). New York: Peter Lang.

Garris, R., Ahlers, R. \& Driskell, J. E. (2002). Games, Motivation and Learning. Research and Practice Model. Simulation \& Gaming, 33(4), 441-467.

Kerres, M., Bormann, M. \& Vervenne, M. (2009). Didaktische Konzeption von Serious Games: Zur Verknüpfung von Spiel- und Lernangeboten. MedienPädagogik. Zeitschrift für Theorie und Praxis der Medienbildung. Online: http://www.medienpaed.com/2009/kerres0908.pdf (05.11.2018).

Lusardi, A. \& Mitchell, O. S., (2011). Financial literacy around the world: an overview. Journal of Pension Economics and Finance, 10(04), 497-50.

Petko, D. (2008). Unterrichten mit Computerspielen. Didaktische Potenziale und Ansätze für den gezielten Einsatz in Schule und Ausbildung. MedienPädagogik: Zeitschrift für Theorie und Praxis der Medienbildung, 15, 1-15.

Rodriguez-Hoyos, C., \& Gomes, M. J. (2012). Beyond the Technological Dimension of Edutainment: An Evaluation Framework with a Curricular Perspective. In M. M. Cruz-Cunha (ed.), Handbook of Research on Serious Games as Educational, Business and Research Tool, (S. 818-837). Hershey, PA: Information Science Reference.

Weinert, F. E. (2001). Vergleichende Leistungsmessung in Schulen - eine umstrittene Selbstverständlichkeit. In F. E. Weinert (Hrsg.), Leistungsmessungen in Schulen, (S. 17-31). Weinheim: Beltz.

Wouters, P., van Nimwegen, C., van Oostendorp, H., \& van der Spek, E. D. (2013). A meta-analysis of the cognitive and motivational effects of serious games. Journal of Educational Psychology, 105(2), 249-265. 


\title{
Mikrodidaktische Planungen von Trainerinnen und Trainern in der betrieblichen Weiterbildung
}

\author{
Tim Stanik
}

\section{Einleitung}

Die betriebliche Weiterbildung ist nach wie vor ein eher vernachlässigtes Forschungsfeld der Berufs- und Wirtschaftspädagogik (vgl. Meyer \& Elsholz 2009). Der Fokus richtet sich vornehmlich auf die berufliche Erstausbildung und insbesondere auf die berufsschulischen Unterrichtspraxen (z. B. Minnamaier, Hermkes \& Mach 2015) sowie auf die Professionalitätsentwicklung der Lehrkräfte (vgl. z. B. Kalisch \& Kaiser 2019).

In Anschluss an Elsholz (2012) wird mit der vorliegenden Studie insofern eine berufs- und wirtschaftspädagogische Perspektive auf betriebliche Weiterbildungen gerichtet, als Unterrichtsvorbereitungen bzw. mikro-didaktische Planungen von Trainerinnen und Trainern (TuT) zum Gegenstand einer explorativen Analyse gemacht werden. Dass nicht die Trainings selbst, sondern die vorbereitenden Planungen untersucht werden, lässt sich u. a. damit begründen, dass diese die Voraussetzung für jede professionelle Lehr- bzw. Unterrichtstätigkeit sind (Peterßen 2000). Aufgrund fehlender Curricula und verbindlicher Lehrbücher in der betrieblichen Weiterbildung scheinen Trainingsplanungen außerdem besonders relevant. Des Weiteren sind sie ein potentieller Ort, an dem pädagogische und betriebliche Handlungslogiken (vgl. Harney 1998) zu verhandeln sind. Zudem kann hierüber ein Zugang zu den pädagogischen Idealen und Auffassungen der Lehrenden eröffnet werden (vgl. Clark \& Yinger 1980).

Mit TuT wird eine Lehrendengruppe untersucht, die häufig als Betriebsexterne auf Basis von Werks- und Honorarverträgen mit der Durchführung der Weiterbildungsmaßnahmen betraut und somit als ein Schlüsselfaktor der betrieblichen Weiterbildungsqualität $\mathrm{zu}$ betrachten ist (vgl. Krekel \& Beicht 1995). Es liegen bislang nur wenige empirische Befunde über das Personal der betrieblichen Weiterbildung und der dort tätigen Lehrenden vor (vgl. Käpplinger \& Lichte 2012). Der WB-Monitor zeigte, dass fast zwei Drittel der Lehrenden, die im Weiterbildungssegment ,privater Markt" tätig werden, einen akademischen Abschluss haben, von denen knapp 15\% einen pädagogischen/erziehungswissenschaftlichen Studienabschluss vorweisen können (vgl. Koscheck 2018). Eine nicht repräsentative Studie kommt zu dem Ergebnis, 
dass $18 \%$ der dort befragten TuT über eine pädagogische Qualifikation verfügen, wobei der Großteil (über 80\%) eine Trainerausbildung absolviert hat (vgl. Fuchs 2011). Ein Aspekt dieser Ausbildungen ist die Trainingsplanung (vgl. Wißhak \& Holdinger 2015). Neben den Trainerausbildungen wird in der Studie von Bonnes und Hochholdinger (2016) auch informellen Lernprozessen eine zentrale Bedeutung zugemessen, um die notwendigen Trainingskompetenzen zu erwerben. Vor der Prämisse, dass es für die Planungen von Trainings professionellen Wissens sowohl in Form von Fachwissen, fachdidaktischen, pädagogisch-psychologischen (vgl. Baumert \& Kunter 2006) als auch technologischen, pädagogischen Wissens (vgl. Koehler \& Mishra 2008) bedarf, stellt sich die Frage, wie Lehrende in diesem formal wenig professionalisierten Feld den didaktischen Anforderungen begegnen und auf welche Konzepte oder Strategien sie dabei zurückgreifen. So eröffnet die empirische Analyse von Trainingsplanungen Zugänge zur Dimension der „Teachers' thought processes" (Clark \& Peterson 1986), in der professionelles Wissen und subjektive Theorien (vgl. Groeben, Wahl, Schlee \& Schlee 1988) ihren Ausdruck finden.

Zunächst wird das untersuchte Phänomen der mikrodidaktischen Planung definiert (2.), und es werden ausgewählte Befunde zur schulischen Unterrichtsbzw. zur außerschulischen Kursvorbereitung skizziert. Hieraus werden die forschungsleitenden Fragestellungen abgeleitet und das qualitative Forschungsdesign begründet (3.). Nach der Darlegung zentraler Ergebnisse (4.), bei der Planungsmotive und -phänomene rekonstruiert werden, schließt der Beitrag mit einem Fazit, in dem drei Ebenen der Trainingsplanung unterschieden sowie Anschlussmöglichkeiten für weitere Forschungen aufgezeigt werden (5.).

\section{Mikrodidaktisches Planungshandeln}

In Anlehnung an das Mehrebenenmodell der betrieblichen Weiterbildungsentscheidungen wird mit den Trainingsplanungen eine spezifische Form mikrodidaktischen Handelns untersucht (vgl. Käpplinger 2016). Gegenstandtheoretisch lassen sich unter Unterrichtsvorbereitungen bzw. unter mikrodidaktischen Planungen alle vorausgehenden Maßnahmen verstehen, die institutionell gerahmte Vermittlungsprozesse im Vorfeld strukturieren (vgl. Gassmann 2013). Präskriptive didaktische Modelle sowohl der schulischen Unterrichtsplanung (vgl. Übersicht bei Jank \& Meyer 2002) als auch erwachsenenpädagogische Konzepte der Kursplanung (vgl. z. B. Schlutz 2006) entwerfen diese mit Hilfe komplexer Strukturmodelle, in denen didaktische Entscheidungsfelder (Lernziele, Lerninhalte, Methoden, Medien, Lernräume), gesellschaftliche und institutionelle Kontextfaktoren sowie Selbstverständnisse der Akteure benannt und deren Interdependenzen aufgezeigt werden. Wenngleich Unterrichtsvorbereitung fester Bestandteil, insbesondere der zweiten Phase der 
Lehramtsausbildung, ist und eine Vielzahl an didaktischen Handreichungen sowohl für die Schule als auch für die Weiterbildung existieren, liegen hierzu vergleichsweise wenig empirische Erkenntnisse vor, sodass die „Bedeutung des Planens unverhältnismäßig reduziert“ (Wernke \& Zierer 2017, 8) werde.

\subsection{Befunde zu Unterrichtsvorbereitungen in der Schule}

Studien der US-amerikanischen Unterrichtsforschung zeigen, dass die Planung der Lerninhalte, Unterrichtsmethoden und die Vorbereitung der Unterrichtsmaterialien zentral sind, wohingegen die Planung von Lernzielen eine untergeordnete Rolle zu spielen scheint (vgl. Shavelson \& Stern 1981). Didaktische Modelle der Unterrichtsplanung werden dabei kaum hinzugezogen (vgl. John 1991; Tebrügge 2001; Seifried 2009), wenngleich auch gezeigt werden konnte, dass die Nutzung von eklektischen Planungsmodellen bei Lehramtsstudierenden dazu führt, sich intensiver mit Lernzielen und der zu unterrichtenden Lernendengruppe auseinanderzusetzen (vgl. Werner, Wernke \& Zierer 2017).

Auch Lehrpläne haben lediglich eine orientierende Funktion. So konnte am Beispiel einer Unterrichtsreihe zur ökonomischen Bildung gezeigt werden, dass ein Drittel der Lehrpersonen den Lehrplan gar nicht bei ihren Unterrichtsplanungen berücksichtigt und die übrigen zwar die hinterlegten Lerninhalte aufgreifen, aber von den dort festgelegten Lernzielen abweichen (vgl. Brinkmann-Herz 1984). Keine eindeutigen Befunde liegen darüber vor, inwiefern Unterrichtsplanungen an den Schüler*innen orientiert sind. In den Studien von Yinger (1980), Bromme (1981) und Haas (1998) spielen sie beispielsweise keine Rolle, wohingegen Sardo-Brown (1990) zeigen konnte, dass erfahrene Lehrer*innen die Interessen und Fähigkeiten der Schüler*innen bei ihren Unterrichtsplanungen berücksichtigen.

Insbesondere die Bedeutung der Lehrerfahrungen konnte in einer Reihe von Vergleichsstudien belegt werden. So planen Novizen z. B. eher einzelne Unterrichtsstunden als Unterrichtseinheiten und entwerfen häufiger schriftliche, detaillierte Unterrichtsentwürfe als ihre erfahrenen Kolleg*innen (vgl. Borko \& Livingston 1989). Seifried (2009) kann für (angehende) Lehrkräfte an kaufmännischen Schulen nachweisen, dass die Planung der Lernziele zugunsten der Lerninhalte mit fortschreitender Lehrerfahrung an Bedeutung gewinnt. Berger und Aprea (2014) unterscheiden in ihrer Studie zwei Typen der Unterrichtsvorbereitung, die zielorientierte und handlungsorientierte Planung, und setzten diese in Bezug zu den planungsüberzogenen Überzeugungen von angehenden Lehrpersonen der beruflichen Bildung. Es konnte sowohl ein positiver Zusammenhang zwischen pädagogisch-didaktischen Motiven mit zielorientierten Planungen als auch zwischen den Nutzenerwartungen von Unterrichtsvorbereitung und einer handlungsorientierten Planung nachwiesen werden. 
Dass Unterschiede in Unterrichtsplanungen sich tatsächlich auf Lehrerfahrungen zurückführen lassen, zeigt eine Längsschnittstudie, in der Lehramtsanwärter*innen aus unterschiedlichen Fächern zwei Jahre begleitet worden sind. Die Planungen wurden zunehmend gröber, sodass die Vorbereitung von Unterrichtseinheiten und nicht mehr von einzelnen -stunden im Vordergrund stand (vgl. Mutton, Hagger \& Burn 2011). Daneben haben die Unterrichtsfächer einen Einfluss auf die Unterrichtsvorbereitungen. So bevorzugen z. B. angehende Mathematiklehrer*innen eher instruktive Lehrmethoden als Lehramtsstudierende des Fachs Erdkunde (vgl. John 1991). Ein zentraler Aspekt der Planung von Mathematiklehrer*innen ist außerdem die Auswahl von Übungsaufgaben aus den Lehrbüchern (vgl. Bromme 1981), wohingegen für Lehrpersonen im Fach Physik die Vorbereitung der Experimente eine zentrale Rolle einnimmt (vgl. Stender 2014). Die Relationierung von Schülervorrausetzungen bei der Planung von Unterrichtsaufgaben (,didaktische Adaptivität“) konnte als Teilfacette der Planungskompetenz von Lehrenden modelliert und anhand von schriftlichen Unterrichtsplanungen gemessen werden. So ließ sich ein positiver Zusammenhang zwischen dieser Facette von Planungskompetenz und einer konstruktivistischen Orientierung der Lehrenden nachweisen (König, Buchholtz \& Dohnen 2015).

\subsection{Befunde zu Kursplanungen in der Erwachsenen-/Weiterbildung}

Mikrodidaktisches Planungshandeln von Lehrenden der betrieblichen Weiterbildung ist zwar nicht explizit untersucht worden, es haben sich aber zwei Studien der Erwachsenenbildungsforschung mit den Kursvorbereitungen der Lehrenden auseinandergesetzt. Außerdem kommt eine weitere Studie zu dem Befund, dass TuT den Trainingsvorbereitungen einen hohen Stellwert beimessen, um den Lerntransfer ihrer Trainings zu sichern.

Haberzeth (2010) rekonstruiert, dass Lehrende in der Erwachsenenbildung Inhalte zum Thema ,Lernen' u. a. mit Hilfe von sach- und erfahrungsbezogenen Strategien aufbereiten, indem sie Lerninhalte danach auswählen, ob diese methodisch gut vermittelbar und anschlussfähig an die Erfahrungen der potenziellen Teilnehmenden sind.

Ein zentrales Ergebnis einer Studie über die subjektiven Wissenskonzeptionen von Lehrenden der Erwachsenenbildung ist die Unterscheidung zwischen „Fachexperten“ und „Methodenexperten“. Während erstere es als ihre Aufgabe betrachten, Sachwissen mit Hilfe lehrzentrierter Unterrichtsmethoden zu vermitteln, wollen die „Methodenexperten“ einen Rahmen schaffen, damit bei den Teilnehmenden Lern- und Bildungsprozesse initiiert werden (vgl. Hof 2001). Dass die didaktischen Planungen auch von den auftraggebenden Organisationen beeinflusst werden, legen weitere Befunde der beiden Studien nahe. So nehmen die Veranstaltungsformen, die Bezahlung der Lehrenden und die 
organisationalen Selbstverständnisse der Weiterbildungseinrichtungen Einfluss auf die jeweiligen Unterrichtskonzeptionen (vgl. Hof 2001). Außerdem erwarten die Auftraggeber in der beruflich-betrieblichen Weiterbildung Nachweise über zielgerichtete Trainings (vgl. Haberzeth 2010). Neben den Lernvoraussetzungen und den Arbeitsumgebungen der Teilnehmenden sichern insbesondere die Gestaltungen der Trainings einen erfolgreichen Lerntransfer (vgl. zur Übersicht Hutchins 2009). Eine qualitative Interviewstudie mit TuT der beruflich-betrieblichen Weiterbildung zeigt, dass diese neben den Gestaltungen der Trainings auch den Aktivitäten vor den Veranstaltungen (z. B. Auftragsklärung mit den Unternehmen, eine an den Betrieb und Teilnehmenden angepasste Trainingskonzeption) eine zentrale Bedeutung für den erfolgreichen Lerntransfer beimessen (vgl. Barth \& Holdinger 2018).

\subsection{Zwischenfazit}

Unter Berücksichtigung der didaktischen Modelle und der empirischen Befunde, sollten im Rahmen von Unterrichtsvorbereitungen bzw. von mikrodidaktischen Trainingsplanungen Entscheidungen über Inhalte, Methoden/Medien, Lernziele, Sozialformen, zeitliche Abfolgen getroffen werden. Diese werden durch Lehrerfahrung, das Selbstverständnis der Lehrenden und durch Unterrichtsfächer bzw. durch Trainingsinhalte beeinflusst. Wenngleich die empirischen Befunde erste Hypothesen bezüglich des mikrodidaktischen Planens von Trainings in der betrieblichen Weiterbildung erlauben, bedarf es zunächst explorativer qualitativer Untersuchungen, um sich folgenden Fragestellungen zu nähern: Wie planen Trainerinnen und Trainer in der betrieblichen Weiterbildung ihre Trainings? Welche didaktischen Entscheidungen werden getroffen? Inwiefern werden diese Entscheidungen miteinander relationiert?

Während die ersten beiden Fragen eine deklarative Ebene der Trainingsvorbereitung fokussieren, zielt die dritte drauf ab, die wechselseitigen Beziehungen zwischen einzelnen didaktischen Planungsaspekten in den Blick zu nehmen und aufzuzeigen, wie diese sich gegenseitig bedingen und zueinander in Bezug gesetzt werden.

\section{Untersuchungsdesign}

An den Continuing Vocational Training Survey anschließend wurden ausschließlich mikrodidaktische Planungen von organisierten Lehr-/Lernprozessen betrachtet, die sich an Mitarbeitende von Betrieben richten, die außerhalb des Arbeitsvollzuges durchgeführt und von den Unternehmen voll- oder teilfi- 
nanziert werden. Mit Trainings wird ein spezieller Typ von Lehrveranstaltungen untersucht, der nicht vornehmlich die Vermittlung von Sachwissen (z. B. Führungstheorien), sondern Änderungen im Verhalten oder der Einstellungen der Teilnehmenden (z. B. Führungsverhalten) zum Ziel hat. Trainings operieren unter den Prämissen, dass sowohl richtige Handlungsweisen existieren als auch dass diese kursförmig zu vermitteln sind (vgl. Hof 2001).

\subsection{Sample der Studie}

Für die Zusammenstellung eines Untersuchungssamples sollten mit Blick auf die empirischen Befunde TuT mit unterschiedlichen Lehrerfahrungen berücksichtigt werden, die aber vergleichbare Trainingsinhalte anbieten. Aufgrund der nicht standardisierten Zugangswege zur Trainingstätigkeit sind außerdem TuT mit unterschiedlichen Qualifikationen einzubeziehen.

Tab. 1: Untersuchungssample

\begin{tabular}{cccclc}
\hline & m/w & Alter & Status & Qualifikation & $\begin{array}{c}\text { Lehrerfahrung } \\
\text { (in Jahren) }\end{array}$ \\
\hline T1 & m & 55 & freiberuflich & Dipl. Psychologie & 25 \\
T2 & m & 53 & freiberuflich & Dipl. Soziale Arbeit (FH) & 21 \\
T3 & w & 33 & festangestellt & $\begin{array}{l}\text { Kommunikationswissen } \\
\text { (BA, MA) }\end{array}$ & 2 \\
T4 & w & 54 & freiberuflich & Lehramt (Anglistik, Sport) & 27 \\
T5 & w & 48 & freiberuflich & Einzelhandelskauffrau (IHK) & 19 \\
T6 & w & 48 & festangestellt & Rhetorik, Kulturwissenschaft, & 11 \\
T7 & w & 59 & freiberuflich & Dipl. Pädagogik & 37 \\
\hline K1 & w & 27 & freiberuflich & Lehramt (Wirtschaft, & 0,5 \\
& & & & Mathematik, DaF) & 35 \\
\hline
\end{tabular}

Quelle: eigene Darstellung

Das Sample der Studie setzt sich aus 5 selbständigen Lehrenden und 2 festangestellten betrieblichen Weiterbildnerinnen zusammen. Die befragten TuT bieten Trainings zu vergleichbaren Themen (z. B. Führung, Team-, Persönlichkeitsentwicklung) an. Die vier selbstständigen TuT (T1, T2, T5, T7) werden als Freiberufler*innen für unterschiedliche Unternehmen und T4 wird als so- 
genannte ,Feste Freie' ausschließlich im Auftrag eines Weiterbildungs-anbieters tätig. Die festangestellte Interviewpartnerin T6 arbeitet als Personalentwicklerin in einem Großunternehmen, und T3 ist als Referentin einer Weiterbildungsakademie eines Wirtschaftsverbandes tätig. Beide führen auch Trainings durch, haben aber mit zwei bzw. elf Jahren deutlich weniger Lehrerfahrungen (Ø 23 Jahre) und weniger Trainingstage pro Jahr als die selbstständigen TuT des Samples. Alle TuT des Samples haben Trainerfortbildungen und bis auf T5 haben alle ein Studium absolviert, davon drei ein pädagogisches/erziehungswissenschaftliches Studium (T2, T4, T7).

Außerdem wurde eine nebenberuflich Lehrende (K1), die Deutsch als Fremdsprache an einer Volkshochschule unterrichtet, und ein weiterer Freiberufler (K2), der Kurse zu philosophischen Themen für konfessionelle Weiterbildungsanbieter anbietet, ins Sample aufgenommen. Hierbei handelt es sich insofern um eine minimale Kontrastierung, als diese Lehrenden andere Themen - kursförmig im Auftrag von Anbietern der allgemeinen Erwachsenenbildung - planen bzw. durchführen.

\subsection{Datenerhebung und-auswertung}

Planungen von Unterricht sind insofern kognitive Entscheidungsprozesse, als Voraussetzungen bei den Lernenden antizipiert, Lerninhalte, -ziele und räumliche Kontextbedingungen berücksichtigt werden und durch das pädagogische Wissen, Erfahrungen und Überzeugungen der Lehrenden beeinflusst werden. Diese bewussten Entscheidungen werden mit zunehmender Lehrerfahrung in mentale Skripte überführt (vgl. Borko, Roberts \& Shavelson 2008). Mikrodidaktische Planungen sind aus diesen Gründen auch nicht mit Hilfe von Beobachtungen zu erfassen und schulische Unterrichtsplanungen werden häufig mit der Methode des lauten Denkens erhoben. Hierbei ist jedoch strittig, inwiefern hinreichende Artikulationsmöglichkeiten bei den Befragten zur Verfügung stehen, um solche komplexen, kognitiven Prozesse während ihres Handelns zu versprachlichen (vgl. Konrad 2010). So merken Shavelson und Stern (1981) an, dass, nur weil Lehrer*innen in den Darstellungen ihrer Unterrichtsplanungen nicht explizit über Lernziele sprechen, dies nicht bedeute, dass diese nicht doch ihre Planungen beeinflussen. Aufgrund dieser methodologischen Probleme wurden die Daten der vorliegenden Studie mit Hilfe problemzentrierter Interviews erhoben (vgl. Witzel 2000). Um dennoch möglichst unvoreingenommene Beschreibungen der Trainingsplanungen zu erhalten, sind die Interviewten zunächst gebeten worden zu beschreiben, wie sie ihre Trainings planen bzw. wie sie ihr letztes Training geplant haben. Erst im Anschluss sind Rückfragen zu didaktischen Entscheidungsfeldern (Inhalte, Methoden, Lernziele, Medien etc.) gestellt worden. Weitere Nachfragen bezogen sich u. a. auf die Gründe für die Planungen. Mit diesem Vorgehen wurden streng genommen 
nicht die Trainingsplanungen, sondern Selbstbeschreibungen über die Trainingsvorbereitungen erfasst.

Die transkribierten Interviews wurden mit Hilfe der Kodierstrategien der Grounded Theory analysiert. Der Prozess des offenen Kodierens wurde durch die Fragestellungen geleitet, welche Handlungs- und Entscheidungsprobleme im Rahmen der Trainingsplanungen angesprochen und wie diese gelöst werden. Anschließend sind die hieraus induktiv entwickelten Codes zu Konzepten „höherer Ordnung“ bzw. zu Phänomenen verdichtet und in ihren Eigenschaften im Prozess des axialen Kodierens mit Hilfe des sogenannten Kodierparadigma dimensionalisiert worden. Dabei wurde zwischen Kontextfaktoren, Strategien, ursächlichen, intervenierenden Bedingungen und Konsequenzen der einzelnen Planungsphänomene differenziert (vgl. Strauss \& Corbin 1996).

\section{Motive und Phänomene der Trainingsplanung}

\subsection{Motive der Trainingsplanung}

Alle Befragten geben an, ihre Trainings zu planen und auch schriftliche Trainingskonzepte zu erstellen. Es lassen sich dabei feldbezogene von persönlichen und didaktischen Motiven unterscheiden. So erwarten die auftraggebenden Unternehmen in der Regel die Vorlage einer schriftlichen Trainingsplanung. In zwei Fällen fungieren die Planungen auch als eigenständiges Produkt, um es an Unternehmen oder an andere Lehrende zu verkaufen (T1, T5). Die drei Freiberufler T1, T2, T3 geben an, dass sie die Planungen auch als Marketinginstrument nutzen, in dem sie diese auf ihre Homepages einstellten, um darüber Trainingsanfragen zu generieren. Die festangestellte Personalentwicklerin T6 und der Freiberufler T1 planen ihre Trainings auch, um mit deren Umsetzungen andere TuT zu beauftragen. Unter die als persönlich kategorisierten Motive lassen sich der Abbau von Unsicherheiten (T3, T6) oder Verweise darauf, dass man ein „strukturierter Typ sei“ (T1, T2), subsumieren. Wenn die Planungen didaktisch begründet werden, steht neben der Relationierung von didaktischen Entscheidungsfeldern (z. B. die Erreichung von Trainingszielen mit bestimmten Methoden), insbesondere die Einhaltung der zeitlichen Vorgaben, die Gewährleistung eines abwechslungsreichen Trainingsablaufs und der Lerntransfer im Vordergrund. 


\subsection{Mikrodidaktische Phänomene der Trainingsplanung}

Insgesamt konnten übergreifend drei Phänomene der Trainingsplanung mit Hilfe der Interviews rekonstruiert werden: die Klärung des Trainingsauftrages, die Auswahl/Planung der Trainingsinhalte und die Planung der Vermittlungsmethoden.

Bis auf die festangestellte Personalentwicklerin (T6) geben alle TuT an, dass ihre Trainingsplanungen mit einem Vorgespräch mit den auftraggebenden Unternehmen beginnen. Dieser Aspekt wird von den beiden Kontrastfällen (K1 und K2) nicht erwähnt. Sie hatten nur einmal Kontakt mit den Vertreter*innen der Weiterbildungseinrichtungen, um dort lehrend tätig zu werden. Im Zentrum der Vorgespräche mit den Betrieben steht die Klärung des Trainingsauftrages. Diese erfolgt im Rahmen von nicht vergüteten Meetings mit den Personal- und/oder Weiterbildungsverantwortlichen der Unternehmen. Ursächliche Bedingungen sind zum einen die Erwartungen der Betriebe an möglichst individualisierte Trainings. Zum anderen habe man die Erfahrung gemacht, dass die angefragten Trainingsthemen zu unspezifisch seien (T1, T3, T7), dass man häufig andere Trainingsinhalte unter die Themen subsumiere (T1, T2, T3) oder dass sich Bedarfe der Teilenehmer*innen von denen der Unternehmen unterscheiden (T1, T3). Ein weiterer zentraler Gesprächspunkt ist die Sicherung des Lerntransfers, der häufig von den Betrieben nicht bedacht werde. Die verfolgten Strategien sind aushandelnd, da hier Trainingsthemen, Ziele der Trainings vor dem Hintergrund der Zielgruppen, der zeitlichen Rahmenbedingungen und der eigenen Trainerexpertise geklärt werden. Zuweilen werden die Gespräche auch auf die potenziellen Teilnehmer*innen ausgeweitet, um deren Bedarfe zu erfassen (T1, T3, T4). Intervenierende Bedingungen sind, ob es sich um einen Erst- oder Folgeauftrag für den Betrieb handelt, ob Verantwortlichkeiten für Weiterbildungen in den Betrieben geklärt sind und inwiefern hier bereits makrodidaktische Entscheidungen getroffen worden sind. Wird der Trainingsauftrag erteilt, werden die hier ausgehandelten Trainingsthemen und -ziele zu ursächlichen Bedingungen, und die Rahmenbedingungen (Zielgruppe, zeitlicher Umfang) fungieren als intervenierende Bedingungen für die weiteren Planungsprozesse.

Danach erfolgt die Auswahl/Planung der Trainingsinhalte durch die TuT. In diesem Zusammenhang werden auch Lernziele angesprochen, wenngleich diese häufig mit den Lerninhalten gleichgesetzt oder sehr allgemein als Vermittlung von Handlungswissen oder mit dem Anstoßen von Reflexionsprozessen beschrieben werden: „is ja das Ziel nen Prozess anzuregen ne gewisse Selbstreflektion" (T4). Lediglich T1 unterscheidet in seinen Darstellungen zwischen kognitiven, affektiven und psychomotorischen Lernzielen. Die Strategien der Ermittlung der Trainingsinhalte sind nicht systematisch, sondern lassen sich als assoziativ charakterisieren. Lerninhalte werden vornehmlich im Internet recherchiert. Weitere Quellen zur Ermittlung der Lerninhalte sind 
Konsultationen von Fachliteratur, eigene Seminarbesuche oder der Austausch mit Kolleg*innen. Die erfahrenen TuT (T1, T2, T5, T7) bedienen sich zudem Copy und Paste Strategien, indem Inhalte aus zurückliegenden Trainings übertragen werden. Intervenierende Bedingung ist sowohl das eigene Fachwissen als auch der Anspruch, nur jene Inhalte zu vermitteln, die für Teilnehmer*innen relevant sind bzw. unmittelbare Bezüge zu ihren beruflichen Aufgaben haben. So werden auch Fragen oder Fallbeispiele überlegt, um Lerninhalte mit den Tätigkeitsfeldern der Teilnehmenden in Beziehung setzen zu können (T2, T5, T6, T7). Daneben nehmen Vorgaben der Unternehmen (z. B. bereits etablierte Kompetenzmodelle) oder antizipiertes Vorwissen der Zielgruppen (z. B. erfahrene Führungskräfte kennen bereits Führungstheorien (T4)) Einfluss auf die Auswahl der Lerninhalte. Die Reduktion der Lerninhalte ist weniger didaktisch orientiert (vgl. Lehner 2013), sondern erfolgt primär strukturell über die zur Verfügung stehenden Trainingszeiten. Im Vergleich zu den beiden Kontrastfällen, werden die Kursinhalte von der DaF Lehrenden (K1) dem Lehrbuch entnommen, und der Kursleiter im Bereich Philosophie (K2) plant lediglich Themen (z. B. „Was ist Gerechtigkeit?“ oder „Was heißt ein erfülltes Leben?"), um hierüber mit seinen Teilnehmenden ins Gespräch zu kommen. Die Konsequenz der Planung bzw. der Auswahl der Lerninhalte ist die Verschriftlichung eines thematischen Strukturplans. Außerdem werden die Lerninhalte zu den ursächlichen Bedingungen für die Planungen der Vermittlungsmethoden.

Die Planung der Vermittlungsmethoden wird von allen Befragten als zentraler Aspekt der Trainingsplanung beschrieben. Die ursächlichen Bedingungen sind die zuvor getroffenen Entscheidungen, da Methoden z. B. dazu dienen sollen, Lerninhalte gemäß der Trainingsziele zu vermitteln („,Hilft die Methode zur reinen Erkenntnis oder zur Erweiterung von Handlungskompetenz?“(T2)). Zudem werden sie dazu genutzt, um einzelne Lerninhalte zu Trainingsblöcken zusammenzufassen. Mit der Planung der Vermittlungsmethoden soll außerdem der Lerntransfer der Trainings ermöglicht bzw. gesichert werden. So werden Transferübungen mit hohem Anwendungsbezug in Form von Plan-, Rollenund Fallbeispielen geplant, oder es werden Reflexionsaufgaben vorbereitet, die in Partnerarbeiten zu bearbeiten sind (T2, T4, T5, T6, T7). Neben diesen didaktisch relationierenden Strategien orientiert man sich auch an eigenen Lehrprinzipien (z. B. nicht länger als 20 Minuten am Stück referieren (T3); nach jedem Input folgt ein kurzes Anwendungsbeispiel (T1)). In der Regel können die TuT aus einem Pool an Methoden schöpfen, die sich bereits in anderen Trainings bewährt haben, so dass auch hier Copy und Paste Strategien zum Einsatz kommen. Wichtig ist allen zudem eine abwechslungsreiche Gestaltung der Trainings mit Hilfe der Methoden. Intervenierende Bedingungen sind auf der einen Seite persönliche Vorlieben der TuT und die zeitlichen Rahmenbedingungen der Trainings. Auf der anderen Seite orientiert sich die Methodenplanung an den Zielgruppen bzw. an den potentiellen Teilnehmer*innen. So 
seien beispielsweise aktivierende Methoden bei Vertrieblern besonders wichtig (T2), Gruppenübungen bei Juristen*innen nicht zielgruppenorientiert (T3) oder Projektmanager*innen würden eher selbstgesteuerte Lehr-/Lernmethoden bevorzugen (T1): „Bestimmte Zielgruppen lechzen gerade nach bestimmten Methoden“" (T1). Während die Trainingsthemen, -inhalte und -ziele nicht mit den Teilnehmenden verhandelbar sind, werden alternative Vermittlungsmethoden geplant, um in den Trainings auch auf Wünsche der Lernenden eingehen zu können. Die Konsequenzen der Methodenplanung sind die Vorbereitungen der Trainingsmaterialien (z. B. Präsentationsfolien, Arbeitsblätter, Lerntagebücher) und schließlich die Erstellung von schriftlichen Trainingsentwürfen - häufig „Trainerdrehbuch“ genannt - die den auftraggebenden Betrieben vor den Trainings auch vorzulegen sind.

\section{Fazit und Ausblick}

Die rekonstruierten mikrodidaktischen Trainingsvorbereitungen bewegen sich an Carle (1995) anschließend und erweiternd auf drei Planungsebenen. Die erste Ebene lässt sich als soziale Prozessebene bezeichnen, da zunächst im persönlichen Austausch mit den Personal- oder Weiterbildungsverantwortlichen die Trainingsaufträge ausgehandelt und konkretisiert werden. Es werden Trainingsthemen, die mit den Lehrveranstaltungen verfolgten Zielsetzungen, die zeitlichen Rahmenbedingungen und die adressierten Zielgruppen festgelegt. Während schulische Unterrichtsplanungen eine Verbindung zwischen Lehrplan und dem Unterricht herzustellen haben (vgl. Kron 2008), werden hier Erwartungen oder bereits gefällte makrodidaktische Weiterbildungsentscheidungen der Betriebe zu den Ausgangspunkten für die weiteren Planungsaktivitäten. Unternehmen im Zuge der Auftragsklärung hinsichtlich des Lerntransfers zu beraten (vgl. Barth \& Holdinger 2018), wird von den hier befragten TuT nicht genannt. Auf einer zweiten, der kognitiven Strategieebene, werden die konkreten Lerninhalte und die Vermittlungsmethoden geplant und zueinander in Beziehung gesetzt. Während die Planung und Aufbereitung der Lerninhalte unsystematisch erfolgen und Lernziele auf einer abstrakten Ebene die Vorbereitungen leiten, können die methodischen Planungen dezidiert beschrieben werden. Die Methodenplanungen dienen zur abwechslungsreichen, zielgruppenorientierten Vermittlung der Lerninhalte und zur Sicherung des Lerntransfers im vorgegebenen zeitlichen Rahmen. Im Rückgriff auf die Studie von Hof (2001) verstehen sich die TuT des Samples vornehmlich als Methodenexperten bzw. sehen sie hier ihre zentrale Expertise. Die dritte Ebene ist eine materiale Planungsebene, auf der die Trainingskonzepte verschriftlicht und Trainingsunterlagen erstellt werden. Sowohl die Auftragsklärungen als auch die schrift- 
lichen Trainingskonzeptionen dienen offensichtlich dazu, im Sinne einer betrieblichen Handlungslogik, die Qualität des Produkts Training und dessen Outcome zu sichern (vgl. Harney 1998). Die Planung der Trainingsmethoden lässt sich dagegen als primär didaktisch charakterisieren, da hierüber Relationierungen der Lerninhalte mit den Zielgruppen und den organisatorischen Rahmenbedingungen der Trainings erfolgen. Außerdem werden sie als zentraler Aspekt dargestellt, um den Lerntransfer zu sichern.

Es fällt insgesamt auf, dass die qualifikatorischen Hintergründe der TuT des Samples keinen Einfluss auf die Planungen zu haben scheinen. So greifen weder die Befragten ohne (T1, T3, T5, T6) noch die mit einem pädagogischen/erziehungswissenschaftlichen Studium (T2, T4, T7) explizit auf didaktische Modelle oder Erkenntnisse der Lehr-/Lernforschung bei ihren Darstellungen zurück. Dass die Darstellungen und Begründungen der Trainingsplanungen vornehmlich auf Erfahrungswissen basieren, könnte die Bedeutung der informellen Kompetenzentwicklung in diesem Feld bestätigen (vgl. Bonnes \& Hochholdinger 2016). Auch könnte dies erklären, warum die Beschreibungen der Trainingsplanungen vornehmlich aus der Perspektive der Lehre und nicht aus der Perspektive des Lernens erfolgen (vgl. Oser, Baeriswyl \& Franz 2001).

Auch wenn die vorliegende qualitative Studie aufgrund der geringen Fallzahl und der Einschränkung auf bestimmte Trainingsinhalte weitreichende Limitationen aufweist, zeigen die explorativen Forschungsergebnisse Anschlussmöglichkeiten für weitere Studien auf: Zunächst könnten die Befunde dazu genutzt werden, um sie im Rahmen von repräsentativen Erhebungen zu verifizieren. Sollte sich dabei beispielsweise bestätigen, dass Planungen von TuT mit und ohne einem pädagogischen/erziehungswissenschaftlichen Studienabschluss sich nicht unterscheiden, wären weitere potentielle Einflussfaktoren zu untersuchen. Erste Befunde zeigen beispielsweise, dass Lehrende der beruflich-betrieblichen Weiterbildung über mehr pädagogisch-psychologisches Wissen verfügen als Lehrende der allgemeinen Erwachsenenbildung (vgl. Marx, Goeze, Kelava \& Schrader 2018). So wäre zu prüfen, inwiefern pädagogisch-psychologisches Wissen (vgl. Shulmann 1986) und/oder planungsbezogene Überzeugungen (vgl. Berger \& Aprea 2014) die Trainingsplanungen (nicht) beeinflussen.

Eine weitere noch unbeantwortete Frage ist, wie die Planungen umgesetzt werden. $\mathrm{Zu}$ analysieren ist, inwiefern sie die Durchführungen der Trainings präformieren und inwiefern diese durch situative Entscheidungen modifiziert werden (müssen). In Anlehnung an bzw. in Erweiterung der Figur des „Reflective Practitioner" (Schön 1983) ist diese Forschungsfrage durch die Parameter ,reflection-before-action“ (didaktisches Planungshandeln) und ,reflection-inaction" (Abgleich zwischen Planungs- und Lehr-Lernhandlungen) professionstheoretisch zurückzubinden.

Da die TuT den Klärungen der Trainingsaufträge mit Vertreter*innen der Betriebe einen zentralen Stellenwert für ihre weiteren Planungen beimessen, 
wäre es schließlich notwendig, diese Auftragsklärungen mit Hilfe von konversationsanalytischen Methoden zu untersuchen, um Gesprächsstrategien der Betriebe und der TuT als einen spezifischen Typus institutioneller Interaktion zu analysieren (Drew \& Heritage 1993).

\section{Literatur}

Baumert, J. \& Kunter, M. (2006). Stichwort: Professionelle Kompetenz von Lehrkräften. Zeitschrift für Erziehungswissenschaft, 9(4), 469-520.

Barth, D. \& Hochholdinger, S. (2018). Wie unterstützen Lehrende den Trainingstransfer von Führungstrainings? Eine qualitative Interviewstudie. Bildungsforschung.org, Ausgabe 1. Online: https://open-journals4.uni-tuebingen.de/ojs/index.php/ bildungsforschung/article/view/236/pdf (04.02.2019).

Berger, P. L. \& Aprea, C. (2014). Unterrichtsplanung und planungsbezogene Überzeugungen angehender Lehrpersonen. Journal für Lehrerinnenbildung, 4(14), 35-40.

Bonnes, C. \& Hochholdinger, S. (2016). Die berufliche Entwicklung und die Wege des Qualifikations- und Kompetenzerwerbs von Lehrenden in der berufsbezogenen Weiterbildung.bwp@Berufs- und Wirtschaftspädagogik - online, Ausgabe 29, 1-18. Online: http://www.bwpat.de/ausgabe29/bonnes_hochholdinger_bwpat29. $\operatorname{pdf}(04.02 .2019)$.

Borko H., Roberts, S. A. \& Shavelson, R. (2008). Teachers' Decision Making. Alan J. Bishop to Today. In P. Clarkson \& N. Presmeg (eds.), Critical Issues in Mathematics Education, (S. 37-67) Boston: VS Springer.

Borko, H. \& Livingston, C. (1989). Cognition and Improvisation: Differences in Mathematics Instruction by Expert and Novice Teachers. American Educational Research Journal, 26(4), 473-498.

Brinkmann-Herz, D. (Hrsg.). (1984). Der Einfluss innovativer Lehrpläne auf die Unterrichtsplanung der Lehrer. Eine entscheidungstheoretische Untersuchung am Beispiel eines Lehrplanes zur ökonomischen Bildung in der Hauptschule. Frankfurt: Lang.

Bromme, R. (Hrsg.). (1981). Das Denken von Lehrern bei der Unterrichtsvorbereitung: Eine empirische Untersuchung zu kognitiven Prozessen von Mathematiklehrern. Weinheim: Beltz.

Carle, U. (Hrsg.). (1995). "Mein Lehrplan sind die Kinder" - Eine Analyse der Planungstätigkeit von Lehrerinnen und Lehrern an Förderschulen. Weinheim: Deutscher Studienverlag.

Clark, C. M. \& Peterson, P. L. (1986). Teachers' thought processes. In M. C. Wittrock (ed.), Handbook of research on teaching, (S. 255-296). New York: Macmillan.

Clark, C. M. \& Yinger, R. J. (1980). The hidden world of teaching: implications of research on teacher planning. Research Series No. 77, East Lansing: Michigan State University.

Drew, P. \& Heritage, J. (eds.). (1993). Talk at work: interaction in institutional settings. Cambridge: University Press. 
Elsholz, U. (2012). Betriebliche Weiterbildung als interdisziplinäres Forschungsfeld Annäherung an eine berufs- und wirtschaftspädagogische Perspektive. In U. Faßhauer, B. Fürstenau \& E. Wuttke (Hrsg.), Berufs-und wirtschaftspädagogische Analysen - aktuelle Forschungen zur beruflichen Bildung, (S. 25-34). Opladen: Budrich.

Fuchs, S. (Hrsg.). (2011). Professionalitätsentwicklung des Weiterbildungspersonals. Tätigkeiten, Kompetenzen und Fortbildung von Trainern in der beruflichen/betrieblichen Weiterbildung. Hamburg: Kovac.

Gassmann, C. (Hrsg.). (2013). Erlebte Aufgabenschwierigkeit bei der Unterrichtsplanung. Eine qualitativ-inhaltsanalytische Studie zu den Praktikumsphasen der universitären Lehrerbildung. Wiesbaden: VS Springer.

Groeben, N., Wahl, D., Schlee, J. \& Scheele, B. (Hrsg.). (1988). Das Forschungsprogramm Subjektive Theorien. Tübingen: Francke.

Haas, A. (Hrsg.). (1998). Unterrichtsplanung im Alltag: Eine empirische Untersuchung zum Planungshandeln von Hauptschul-, Realschul- und Gymnasiallehrern. Regensburg: Roderer.

Haberzeth, E. (Hrsg.). (2010). Thematisierungsstrategien im Vermittlungsprozess. Empirische Analysen zum Umgang mit Wissen im Planungsprozess von Weiterbildungsangeboten. Baltmannsweiler: Schneider.

Harney, K. (Hrsg.). (1998). Handlungslogik betrieblicher Weiterbildung. Stuttgart: Hirzel.

Hof, C. (Hrsg.). (2001). Konzepte des Wissens: eine empirische Studie zu den wissenstheoretischen Grundlagen des Unterrichts. Bielefeld: W. Bertelsmann.

Hutchins, H. M. (2009). In the trainer's voice: A study of training transfer practices. Performance Improvement Quarterly, 22(1), 69-93.

Jank, W. \& Meyer, H. (Hrsg.). (2002). Didaktische Modelle: Berlin: Cornelsen.

John, P. D. (1991). A qualitative study of British student teachers' lesson planning perspectives. Journal of Education for Teaching, 17(3), 301-320.

Kalisch, C. \& Kaiser, F. (Hrsg.). (2019). Bildung beruflicher Lehrkräfte - Wege in die pädagogische Königsklasse. Bielefeld: W. Bertelsmann.

Käpplinger, B. (Hrsg.). (2016). Betriebliche Weiterbildung aus der Perspektive von Konfigurationstheorien. Bielefeld: W. Bertelsmann.

Käpplinger B. \& Lichte, N. (2012). Erhöhung der Weiterbildungsbeteiligung durch professionelles Weiterbildungspersonal. WSI-Mitteilungen, (5), 374-381.

König, J., Buchholtz, C. \& Dohnen, D. (2015). Analyse von schriftlichen Unterrichtsplanungen: Empirische Befunde zur didaktischen Adaptivität als Aspekt der Planungskompetenz angehender Lehrkräfte. Zeitschrift für Erziehungswissenschaft, $18(2), 375-404$.

Koehler, M. J. \& Mishra, P. (2008). Introducing TPCK. In AACTE Committee on Innovation \& Technology (ed.), Handbook of technological pedagogical content knowledge for educators, (pp. 3-29). New York, NY: Routledge.

Konrad, K. (2010). Lautes Denken. In G. Mey \& K. Mruck (Hrsg.), Handbuch Qualitative Forschung in der Psychologie, (S. 476-490). Wiesbaden: VS Springer.

Koscheck, S. (2018). Pädagogische Professionalität in Teilsegmenten der Weiterbildung. In Dobischat, R., Elias, A. \& Rosendahl (Hrsg.), Das Personal in der Weiterbildung. Im Spannungsfeld von Professionsanspruch und Beschäftigungsrealität, (S. 161-181). Wiesbaden: VS Springer. 
Krekel, E. M. \& Beicht, U. (1995). Lehrkräfte als Schlüsselfaktor der Weiterbildungsqualität. In R. von Bardeleben, D. Gnahs, E. Krekel \& B. Seusing (Hrsg.), Weiterbildungsqualität - Konzepte, Instrumente und Kriterien, (S. 137-149). Bielefeld: W. Bertelsmann.

Kron, F. W. (Hrsg.). (2008). Grundwissen Didaktik. München: Reinhard.

Lehner, M. (Hrsg.). (2006). Viel Stoff - wenig Zeit: Wege aus der Vollständigkeitsfall. Bern u. a.: Haupt Verlag.

Marx, C., Goeze, A., Kelava, A. \& Schrader, J. (2018). Lehrkräfte in der Erwachsenenund Weiterbildung: Zusammenhänge zwischen Vorbildung und Erfahrung mit dem Wissen über Lehr-Lernmethoden. Zeitschrift für Weiterbildungsforschung, 41(1), 57-77. Online: https://link.springer.com/content/pdf/10.1007\%-2Fs40955018-0108-6.pdf.

Meyer, R. \& Elsholz, U. (2009). Berufliche und betriebliche Weiterbildung als Gegenstand der Berufs- und Wirtschaftspädagogik - Desiderata und neue Perspektiven für Theorie und Forschung.bwp@Berufs- und Wirtschaftspädagogik - online, Ausgabe 16, 1-15. Online: http://www.bwpat.de/ ausgabe16/ meyer_elsholz_bw pat16.pdf (04.02.2019).

Mutton, T., Hagger, H. \& Burn, K. (2011). Learning to plan, planning to learn: the developing expertise of beginning teachers. Teachers and Teaching, 17(4), 399416.

Minnamaier, G., Hermkes, R. \& Mach, H. (2015). Kognitive Aktivierung und Konstruktive Unterstützung als Prozessqualitäten des Lehrens und Lernens. Zeitschrift für Pädagogik, 61(6), 837-856.

Oser, F., Baeriswyl. K \& Franz, J. (2001). Choreographies of Teaching: Bridging Instruction to Learning. In V. Richardson (ed.), Handbook of Research on Teaching, (pp. 1031-1065). Washington: American Educational Research Association.

Peterßen, W. H. (Hrsg.). (2000). Handbuch der Unterrichtsplanung. Grundfragen, Modell, Stufen, Dimensionen. München: Ehrenwirth.

Sardo-Brown, D. (1990). Experienced teachers' planning practices: A US survey. Journal of Education for Teaching, 16(1), 57-71.

Schlutz, E. (Hrsg.). (2006). Bildungsdienstleistung und Angebotsentwicklung. Münster, New York, München: Waxmann.

Schön, D. (ed.). (1983). The Reflective Practitioner: How professionals think in action. London: Basic Books.

Shavelson, R. J. \& Stern, P. (1981). Research on Teachers' Pedagogical Thoughts, Judgments, Decisions, and Behavior. Review of Educational Research, 51(4), 455498.

Shulman, L. S. (1986). Those who understand: Knowledge growth in teaching. Educational Researcher, 15(2), 4-14.

Seifried, J. (2009). Unterrichtsplanung von (angehenden) Lehrkräften an kaufmännischen Schulen. Zeitschrift für Berufs- und Wirtschaftspädagogik, 105(2), 179197.

Stender, A. (Hrsg.). (2014). Unterrichtsplanung: Vom Wissen zum Handeln - Theoretische Entwicklung und empirische Überprüfung des Transformationsmodells der Unterrichtsplanung. Berlin: Logos.

Strauss, A. L. \& Corbin, J. (Hrsg.). (1996). Grounded Theory: Grundlagen Qualitativer Sozialforschung. Weinheim: Beltz. 
Tebrügge, A. (Hrsg.). (2001). Unterrichtsplanung zwischen didaktischen Ansprüchen und alltäglicher Berufsanforderung: Eine empirische Studie zum Planungshandeln von Lehrerinnen und Lehrern in den Fächern Deutsch, Mathematik und Chemie. Frankfurt: Peter Lang.

Werner, J., Wernke, S. \& Zierer, K. (2017). Der Einfluss didaktischer Modelle auf die allgemeindidaktische Unterrichtskompetenz von Lehramtsstudierenden. In S. Wernke \& K. Zierer (Hrsg.), Die Unterrichtsplanung - Ein in Vergessenheit geratendener Kompetenzbereich?! (S. 104-120). Bad Heilbrunn: Klinkhardt.

Wernke, S. \& Zierer, K. (2017). Die Unterrichtsplanung - Ein in Vergessenheit geratendener Kompetenzbereich?! In S. Wernke \& K. Zierer (Hrsg.), Die Unterrichtsplanung - Ein in Vergessenheit geratendener Kompetenzbereich?! (7-16). Bad Heilbrunn: Klinkhardt.

Wißhak, S. \& Hochholdinger, S. (2015). „Zaubern“ lernen - Welche pädagogischen Inhalte umfassen sogenannte Trainerausbildungen? Zeitschrift für Weiterbildungsforschung -Report 38, 113-127. Online: https://www.die-bonn.de/doks/zfw/2015professionalitaet-01.pdf. (21.03.2019).

Witzel, A. (2000). Das problemzentrierte Interview. Forum Qualitative Sozialforschung/Forum qualitative Social Research, 1(1). Online: http://www.qualitativeresearch.net/fqs-texte/1-00/1-00witzel-d.pdf. (04.02.2019).

Yinger, R. J. (1980). A study of teacher planning. The Elementary School, 80(3), $107-127$. 


\section{Herausgeberschaft}

Prof. Dr. Eveline Wittmann

Technische Universität München

Lehrstuhl für Berufspädagogik

eveline.wittmann@tum.de

Prof. Dr. Dietmar Frommberger

Universität Osnabrück

Professur für Berufs- und Wirtschaftspädagogik

dietmar.frommberger@uni-osnabrueck.de

Prof. Dr. Ulrike Weyland

Westfälische Wilhelms-Universität Münster

Professur für Erziehungswissenschaft

Schwerpunkt Berufspädagogik

ulrike.weyland@uni-muenster.de

\section{Autorinnen und Autoren}

Prof. Dr. Carmela Aprea

Universität Mannheim

Lehrstuhl für Wirtschaftspädagogik

Design und Evaluation instruktionaler Systeme

aprea@bwl.uni-mannheim.de

Marc Egloffstein, Dipl. Hdl., Dipl.-Wirtsch. Inf.

Universität Mannheim

Lehrstuhl für Wirtschaftspädagogik

Technologiebasiertes Instruktionsdesign

egloffstein@uni-mannheim.de

Anja Frim, M.Sc.

Otto-Friedrich-Universität Bamberg

Studentin der Wirtschaftspädagogik 
Tobias Heilig, M.Sc.

Universität Mannheim

Lehrstuhl für Wirtschaftspädagogik

Technologiebasiertes Instruktionsdesign

tobias_heilig@gmx.de

Prof. Dr. Karin Heinrichs

Pädagogische Hochschule Österreich

Professur für Lehren und Lernen im Beruf

karin.heinrichts@ph-oot.at

Prof. Dr. Dirk Ifenthaler

Universität Mannheim

Lehrstuhl für Wirtschaftspädagogik

Technologiebasiertes Instruktionsdesign

ifenthaler@uni-mannheim.de

Jun.-Prof. Dr. Tobias Kärner

Universität Konstanz

Fachbereich Wirtschaftswissenschaften

Juniorprofessur für Wirtschaftspädagogik

Schwerpunkt Heterogenität

tobias.kaerner@uni-konstanz.de

Prof. Dr. Franz Kaiser

Universität Rostock

Institut für Berufspädagogik

franz.kaiser@uni-rostock.de

Thilo J. Ketschau, Dipl. Päd., MBA

Technische Universität Dortmund

Institut für Berufspädagogik

thilo.ketschau@tu-dortmund.de

Prof. Dr. Marc Kleinknecht

Professur für Schulpädagogik und Schulentwicklung

Leuphana Universität Lüneburg

marc.kleinknecht@leuphana.de

Prof. Dr. Rer. Soc. Karin Reiber

Hochschule Esslingen

Fakultät für soziale Arbeit, Gesundheit und Pflege

karin.reiber@hs-esslingen.de 
Hannes Reinke, M. Sc.

Otto-Friedrich-Universität Bamberg Professur für Wirtschaftspädagogik hannes.reinke@uni-bamberg.de

Julia Schultheis, M.Sc.

Universität Mannheim

Lehrstuhl für Wirtschaftspädagogik

Design und Evaluation instruktionaler Systeme

schultheis@bwl.uni-mannheim.de

Dr. Tim Stanik

Eberhard Karls Universität Tübingen

Institut für Erziehungswissenschaft

Abteilung Erwachsenenbildung/Weiterbildung

tim.stanik@uni-tuebingen.de

Prof. Dr. Bärbel Wesselborg

Fliedner Fachhochschule Düsseldorf

Lehrerprofessionalität in der beruflichen Fachrichtung Pflege

wesselborg@fliedner-fachhochschule.de

Prof. Dr. Ulrike Weyland

Westfälische Wilhelms-Universität Münster

Professur für Erziehungswissenschaft

Schwerpunkt Berufspädagogik

ulrike.weyland@uni-muenster.de

Prof. Dr. Eveline Wittmann

Technische Universität München

Lehrstuhl für Berufspädagogik

eveline.wittmann@tum.de 


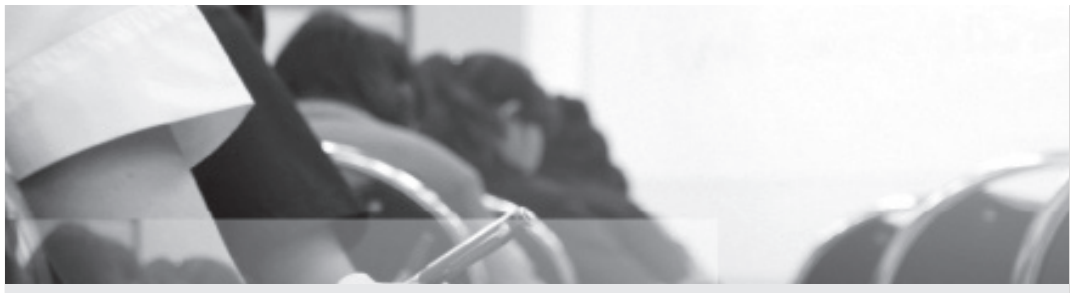

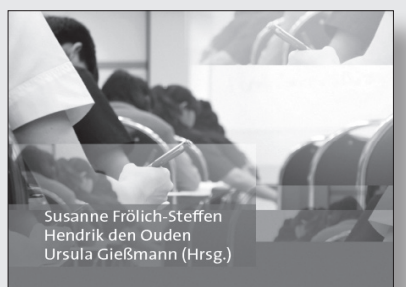

Kompetenzorientiert

prüfen und bewerten an

Universitäten

Didaktische Grundannahmen,

rechtliche Rahmenbedingungen und

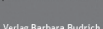

Vefloge Barbara Budrich

B:
Susanne Frölich-Steffen

Hendrik den Ouden

Ursula Gießmann (Hrsg.)

Kompetenzorientiert prüfen und bewerten an Universitäten

2019• 197 Seiten $\cdot$ Kart. $\cdot 17,90 €(D) \cdot 18,40 €(A)$

ISBN 978-3-8474-2294-5 • eISBN 978-3-8474-1473-5

Prüfungen sind ein Kernstück akademischer Ausbildungen. Sie zeigen die erworbenen Kompetenzen, reihen die Studierenden nach Leistungen und sind für jede Studiengangsorganisation unerlässlicher Bestandteil der Studienplanung. Dieser zentralen Bedeutung akademischer Prüfungen steht ein eklatanter Mangel an Fachliteratur zur Konzeption, Durchführung und Bewertung der Prüfungsformate gegenüber, die im Hochschulalltag aufzufinden oder denkbar sind. Genau diese Lücke soll dieses Buch schließen.

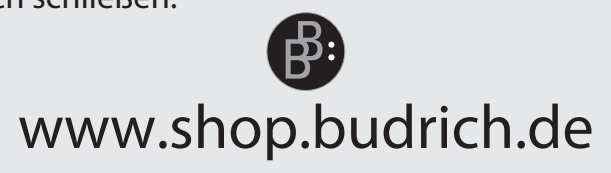




\section{E. Wittmann, D. Frommberger, U. Weyland (Hrsg.)}

\section{Jahrbuch der berufs- und \\ wirtschaftspädagogischen Forschung 2019}

Das Jahrbuch für Berufs- und Wirtschaftspädagogik gibt einen Überblick über den aktuellen Stand einschlägiger Forschung. Mit diesem Zuschnitt wird dem breiten thematischen und methodologischen Spektrum der Forschung und Theoriebildung in der Berufs- und Wirtschaftspädagogik Rechnung getragen. Der diesjährige Band versammelt Beiträge, die bei der Tagung der Sektion Berufs- und Wirtschaftspädagogik der DGfE erstmalig einer breiteren Fachöffentlichkeit präsentiert wurden. Die Beiträge wurden einem Reviewverfahren unterzogen.

Die Herausgeber*innen:

Prof. Dr. Eveline Wittmann, Lehrstuhl für Berufspädagogik, Technische Universität München

Prof. Dr. Dietmar Frommberger, Professur für Berufs- und Wirtschaftspädagogik, Universität Osnabrück

Prof. Dr. Ulrike Weyland, Professur für Erziehungswissenschaft, Westfälische Wilhelms-Universität Münster 